Sistemas de Controle Lineares em Grupos de Lie

\author{
Ana Carolina Dias do Amaral Ramos
}




\title{
Sistemas de Controle Lineares em Grupos de Lie
}

\author{
Ana Carolina Dias do Amaral Ramos
}

Orientador: Prof. Dr. Eyüp Kızıl

Dissertação apresentada ao Instituto de Ciências Matemáticas e de Computação - ICMC-USP, como parte dos requisitos para obtenção do título de Mestre em Ciências- Matemática. VERSÃO REVISADA.

\author{
USP - São Carlos \\ Julho/2013
}


Ficha catalográfica elaborada pela Biblioteca Prof. Achille Bassi e Seção Técnica de Informática, ICMC/USP, com os dados fornecidos pelo(a) autor(a)

Dias do Amaral Ramos, Ana Carolina

Sistema de controle lineares em grupos de Lie / Ana Carolina Dias do Amaral Ramos; orientador Eyüp Kizil. -- São Carlos, 2013.

$104 \mathrm{p}$.

Dissertação (Mestrado - Programa de Pós-Graduação em Matemática) - - Instituto de Ciências Matemáticas e de Computação, Universidade de São Paulo, 2013.

1. Sistemas de controle. 2. Grupos de Lie. 3. Normalizador. 4. Controlabilidade. 5.

Transitividade. I. Kizil, Eyüp, orient. II. Título. 

1 Preliminares $\quad 17$

1.1 Variedades Diferenciáveis . . . . . . . . . . . . . . . . . . . . 17

1.2 Espaço Tangente . . . . . . . . . . . . . . . . . . . . . . . . . 19

1.3 Campos de Vetores em Variedades . . . . . . . . . . . . . . . . . 21

1.4 Curvas integrais e o Fluxo Local . . . . . . . . . . . . . . . . . . . . . . . . . . . . . 24

1.5 Sistemas de Controle em Variedades . . . . . . . . . . . . . . . . . 29

2 Grupos de Lie e Suas Álgebras de Lie $\quad 35$

2.1 Grupos de Lie . . . . . . . . . . . . . . . . . . . . . . . . 35

2.2 Álgebra de Lie de um Grupo de Lie . . . . . . . . . . . . . . . . . . . . . . . . . . . 39

2.3 Derivações e Produtos Semidiretos . . . . . . . . . . . . . . . . . . . . . . 42

2.4 Campos Invariantes . . . . . . . . . . . . . . . . . . . . . . 46

2.5 Aplicação Exponencial . . . . . . . . . . . . . . . . . . . . . . 49

3 Sistemas Lineares em $\mathbb{R}^{n} \quad \mathbf{5 3}$

3.1 Exemplos . . . . . . . . . . . . . . . . . . 53

3.2 Condição do Posto de Kalman . . . . . . . . . . . . . . . . . . . . 55

3.3 Matriz de Controlabilidade . . . . . . . . . . . . . . . 61

4 Sistemas de Controle Lineares em Grupos de Lie $\quad 67$

4.1 Motivação . . . . . . . . . . . . . . . . . . . . . . . . . . . . . . . . .

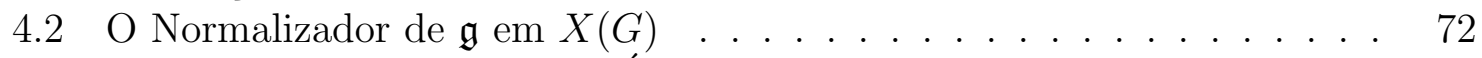

4.3 Automorfismos de Grupos e Álgebras de Lie . . . . . . . . . . . . . . . 74

4.4 Identificação de Campos com Funções . . . . . . . . . . . . . . . . . . . 78

4.5 Transitividade . . . . . . . . . . . . . . . . . . . . 87

4.6 Controlabilidade Local . . . . . . . . . . . . . . . . . . . 95

$\begin{array}{ll}\text { Índice Remissivo } & 103\end{array}$ 



\section{Agradecimentos}

Agradeço em primeiro lugar a minha mãe pelo apoio incondicional, por fazer-se presente todo o tempo, e por seu exemplo de perseverança.

À minha irmã, que é a minha alegria.

À minha amiga Bruna, por me compreender, pelos conselhos e paciência.

Aos amigos do Mestrado, Di, Evandro, Victor, Laís e Gabriel, com os quais pude dividir angústias e alegrias dessa jornada.

Ao Apoenã pelo companherismo.

Aos meus afilhados Gre e Renato por me darem suporte.

À família Silva, da qual passei a fazer parte.

Aos amigos conquistados em São Carlos, Andreza, Mayron, Thaís, Carlos, Karina, Felipe, Marvin.

Ao meu orientador, Eyüp Kızıl, pela dedicação e esforço para que este trabalho fosse concluído.

Por fim agradeço a CAPES pelo apoio financeiro. 

Estudamos sistemas lineares em grupos de Lie introduzido por Ayala e Tirao em [3]. Esta nova classe de sistemas de controle é obtido através de uma generalização aos grupos de Lie de campos de vetores lineares em espaços vetoriais. Eles extendem não somente a classe bem conhecida de sistemas lineares em $\mathbb{R}^{n}$ mas também sistemas invariantes em grupos de Lie e os avanços recentes mostram que eles aparecem como modelos para ampla classe de sistemas de controle proveniente de diversas áreas de ciência e engenharia.

Focamos nossa atenção em normalizador, que tem tido um papel fundamental em formulação de sistemas lineares em grupos de Lie, e lidamos com curvas integrais de seus campos vetoriais.

Finalmente mostramos que sob certas hipóteses sistemas lineares em grupos de Lie possuem a propriedade de controlabilidade local a partir de identidade do grupo. 

We study linear control systems on Lie groups introduced by Ayala and Tirao in [3]. This new class of control systems is obtained through a generalization to Lie groups of linear vector fields on vector spaces. They extend not only well-known class of linear control systems on $\mathbb{R}^{n}$ but also invariant systems on Lie groups and recent achievements show that they appear as models for a wide class of control systems coming from several areas of science and engineering.

We focus our attention on the notion of normalizer which has been played a key role for formulation of linear systems on Lie groups and then deal with integral curves of its vector fields.

Finally we show that under certain assumptions linear systems on Lie groups have local controllability property from the group identity. 

Sistemas lineares em grupos de Lie no contexto da teoria de controle foram considerados primeiro por L. Markus em [15] sobre grupos de Lie de matrizes. E posteriormente no caso geral por Ayala e Tirao em [3]. Esta nova classe de sistemas é obtida generalizando a grupos de Lie os campos de vetores lineares em espaços vetoriais. Na verdade, são sistemas não-lineares mas por essa razão ainda são chamados de lineares. Esse trabalho consiste então principalmente no estudo do artigo de Ayala-Tirao.

Por definição, um sistema de controle linear é determinado pelo par

$$
\Sigma=(G, \mathcal{D})
$$

onde o espaço estado é um grupo de Lie conexo $G$ de dimensão finita cujá álgebra de Lie é $\mathfrak{g}$ e $\mathcal{D}$ é a dinâmica dada pela seguinte família de equações diferenciais ordinárias em $G$

$$
\dot{x}(t)=X(x)+\sum_{j=1}^{m} u_{j} Y^{j}(x), \quad x \in G,
$$

parametrizadas por controles admissíveis $u \in \mathcal{U}_{m}$. O campo $X$ livre de controle é um campo vetorial linear, isto é, seu fluxo é um subgrupo a 1-parâmetro de automorfismos e os campos $Y^{j}$ todos pertencem à álgebra de Lie $\mathfrak{g}$ de campos invariantes à esquerda.

A importância de sistemas lineares em grupos de Lie é que eles generalizam não somente sistemas lineares em $\mathbb{R}^{n}$ mas também sistemas invariantes em grupos de Lie que recebeu notável atenção de teoristas de controle desde 1960. Uma revisão completa de mais de 40 anos de pesquisa sobre sistemas invariantes em grupos de Lie pode ser encontrada em [20].

Graças a Teorema de equivalência obtido recentemente por P. Jouan em [9], sabemos agora que sistemas lineares em grupos de Lie podem ser generalizados a espaços homogêneos e aparecem como prototipo para uma classe mais ampla de sistemas de controle. 
A dissertação está dividida em quatro capítulos.

No Capítulo 1, introduzimos os conceitos básicos da geometria diferencial moderna tais como variedades diferenciáveis, espaço tangente, campos de vetores e seus fluxos, colchete de Lie de campos, etc. Ao final do capítulo, encontra-se uma breve exposição sobre sistemas de controle em variedades com o intuito de preparar o leitor para capítulos subsequentes.

No Capítulo 2 iremos nos apropriar das definições, propriedades e alguns resultados clásicos da teoria de grupos e álgebras de Lie.

No Capítulo 3, encontra-se a principal motivação para o estudo de sistemas lineares em grupos de Lie. O capítulo traz definições e exemplos de sistemas lineares em $\mathbb{R}^{n}$ e dá ênfase ao teorema de Kalman que caracteriza controlabilidade completa. Um sistema de controle linear $\Sigma$ em $\mathbb{R}^{n}$ é da forma

$$
\dot{x}(t)=A x(t)+B u(t)
$$

onde $A$ e $B$ são matrizes de dimensões apropriadas e o parâmetro $u$ pertence a classe de controles constantes por pedaços. Esta é, certamente, a classe mais importante de sistemas tanto por um ponto de vista teórico quanto prático. Dado um sistema linear $\Sigma$ em $\mathbb{R}^{n}$ podemos associar a $\Sigma$ uma matriz $n \times n m$

$$
M=\left(\begin{array}{lllll}
B & A B & A^{2} B & \cdots & A^{n-1} B
\end{array}\right)
$$

conhecida como matriz de controlabilidade. Kalman, Ho e Narendra provaram em [13] que $\Sigma$ é controlável se, e somente, se o posto da matriz $M$ coincide com a dimensão de $\mathbb{R}^{n}$.

No último Capítulo 4, tratamos do estudo de sistemas de controle lineares em grupos de Lie, o objetivo central desta dissertação. Esta nova classe de sistemas foi introduzida na literatura por Ayala-Tirao, [3], através de noção de normalizador que também está presente em sistemas lineares em espaços Euclideanos. Os elementos de normalizador $\mathfrak{n}_{X(G)}(\mathfrak{g})$ são campos de vetores lineares, ou seja, campos cujos fluxos são automorfismos de $G$.

A caracterização de normalizador como um produto semi-direto de álgebras de Lie é dada no Teorema 4.2.3. Mais precisamente, se $G$ é um grupo de Lie conexo com álgebra de Lie $\mathfrak{g}$ então

$$
\mathfrak{n}_{X(G)}(\mathfrak{g}) \cong \mathfrak{g} \otimes \mathfrak{a} \mathfrak{u t}(G)
$$

onde $\mathfrak{a u t}(G)$ é a álgebra de Lie do grupo $\operatorname{Aut}(G)$ de automorfismos de $G$. Em particular, se $G$ é conexo e simplesmente conexo então $\mathfrak{n}_{X(G)}(\mathfrak{g}) \cong \mathfrak{g} \otimes \mathfrak{D} \mathfrak{e r}(\mathfrak{g})$ onde $\mathfrak{D} \mathfrak{e r}(\mathfrak{g})$ significa a álgebra de Lie de todas as derivações de $\mathfrak{g}$. 
Usando uma identificação útil de campos de vetores com funções diferenciáveis definidas em $G$ a valores em $\mathfrak{g}$ mostra-se quando um campo $X$ em $X(G)$ induzido por uma função $F \in C^{\infty}(G, \mathfrak{g})$ pertence ao normalizador $\mathfrak{n}_{X(G)}(\mathfrak{g})$. Com isso será possível ver explicitamente a cara de soluções de um sistema de controle linear sobre um grupo de Lie conexo. Veja Teorema 4.4.6.

Abordaremos também condições de natureza algébrica que dão informação sobre transitividade de sistemas lineares em grupos de Lie. Em particular, mostramos que a álgebra de Lie do sistema é um produto semi-direto de álgebras de Lie. Finalmente, apresentaremos um resultado de controlabilidade local na identidade para esta classe de sistemas. 



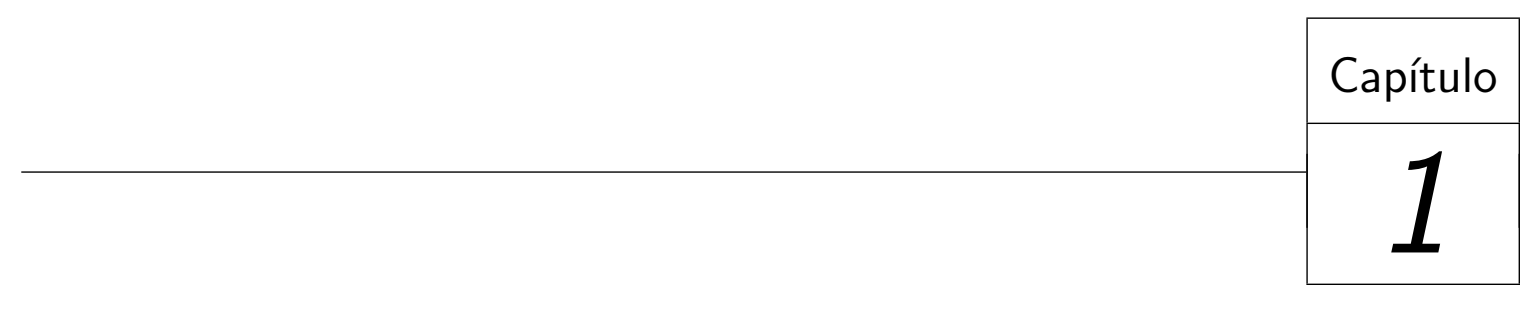

Preliminares

Pretendemos nesse capítulo dar uma breve introdução aos principais conceitos usados no decorrer deste texto, tais como, variedades diferenciáveis, campos vetoriais, curvas integrais, fluxo de um campo, procurando fornecer o minímo necessário para uma boa compreensão dos resultados dos capítulos a seguir. Este capítulo introdutório em sua grande parte foi baseado nas notas de aula de [16].

\subsection{Variedades Diferenciáveis}

Definição 1.1.1. Seja $M$ um conjunto. Uma carta local em $M$ é uma bijeção $\varphi: U \rightarrow$ $\varphi(U)$, onde $U$ é um subconjunto de $M$ e $\varphi(U)$ é um aberto de algum espaço Euclidiano $\mathbb{R}^{n}$. Duas cartas locais em $M, \varphi: U \rightarrow \varphi(U)$ e $\psi: V \rightarrow \psi(V)$, são $C^{k}$-compatíveis $(0 \leq k \leq \infty)$ se $\varphi(U \cap V)$ e $\psi(U \cap V)$ são abertos em $\mathbb{R}^{n}$ e a aplicação de transição $\psi \circ \varphi^{-1}$ é um difeomorfismo de classe $C^{k}$.

Definição 1.1.2. Um atlas $\mathcal{A}$ de classe $C^{k}$ e dimensão $n$ em um conjunto $M$ é uma coleção $\mathcal{A}=\left\{\left(U_{\alpha}, \varphi_{\alpha}\right): \alpha \in I\right\}$ de cartas locais em $M$, onde cada $\varphi_{\alpha}\left(U_{\alpha}\right)$ é aberto em $\mathbb{R}^{n}$, duas a duas $C^{k}$-compativeis, e tal que $M=\cup_{\alpha \in I} U_{\alpha}$. Um atlas $\mathcal{A}$ de classe $C^{k} e ́$ dito maximal se não está propriamente contido em nenhum outro atlas de classe $C^{k}$ em $M$.

Dadas uma carta local $\varphi$ e um atlas $\mathcal{A}$ de classe $C^{k}$ em $M$ diremos que $\varphi$ é $C^{k}$ compatível com $\mathcal{A}$ se ela é $C^{k}$-compatível com toda carta $\psi \in \mathcal{A}$. Note que se $\mathcal{A}$ é um 
atlas de classe $C^{k}$ em $M$ e, $(U, \varphi)$ e $(V, \psi)$ são cartas locais em $M$, ambas $C^{k}$-compatíveis com $\mathcal{A}$, então $\varphi$ e $\psi$ são $C^{k}$-compatíveis.

Definição 1.1.3. Uma variedade diferenciável de classe $C^{k}$ e dimensão n é um par $(M, \mathcal{A})$, onde $M$ é um conjunto e $\mathcal{A}$ é um atlas maximal de classe $C^{k}$ e dimensão $n$ em $M$ tal que a topologia induzida em $M$ por $\mathcal{A}$ é Hausdorff e satisfaz o segundo axioma da enumerabilidade.

Exemplos 1.1.4. 1. O conjunto unitário $\mathcal{A}=\left\{\left(\mathbb{R}^{n}, \mathrm{Id}\right)\right\}$ é um atlas de classe $C^{\infty}$ em $\mathbb{R}^{n}$ uma vez que a aplicação identidade Id é um homeomorfismo. O atlas maximal $\mathcal{A}_{\text {max }}$ que contém $\mathcal{A}$ consiste de todos os difeomorfismos $\varphi: U \rightarrow \varphi(U)$ de classe $C^{\infty}$, com $U$ e $\varphi(U)$ sendo abertos de $\mathbb{R}^{n}$.

2. O conjunto $\mathcal{A}$ que consiste de todos os isomorfismos lineares $\varphi: V \rightarrow \mathbb{R}^{n}$ de um espaço vetorial real $V$ é um atlas de classe $C^{\infty}$ em $V$. Portanto, o espaço vetorial $V$, munido do altas maximal quew contém $\mathcal{A}$, é uma variedade diferenciável de classe $C^{\infty}$.

3. Todo aberto $W$ de uma variedade diferenciável $M^{n}$ de classe $C^{k}$ é também uma (sub)variedade de mesma classe e dimensão.

Discutiremos agora brevemente a noção de diferenciabilidade de aplicações entre variedades diferenciáveis.

Definição 1.1.5. Sejam $M^{m}, N^{n}$ duas variedades diferenciáveis de classe $C^{k}$. Dizemos que uma aplicação $f: M \rightarrow N$ é de classe $C^{r}, 1 \leq r \leq k$, se para todo $p \in M$, existem cartas locais $\varphi: U \rightarrow \varphi(U)$ em $M$ e $\psi: V \rightarrow \psi(V)$ em $N$ tais que $p \in U$, $f(U) \subset V$ e $\psi \circ f \circ \varphi^{-1}$ seja de classe $C^{r}$.

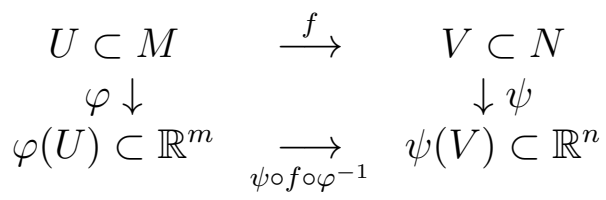

Note que a composta $\psi \circ f \circ \varphi^{-1}$ é a aplicação que representa $f$ em relação às cartas $\varphi$ e $\psi$, e a definição acima independe da escolha das cartas.

Definição 1.1.6. Uma aplicação $f: M \rightarrow N$ é um difeomorfismo de classe $C^{k}$ se $f$ é uma bijeção de classe $C^{k}$, cuja inversa $f^{-1}: N \rightarrow M$ também é de classe $C^{k}$. A aplicação $f$ é chamada de um difeomorfismo local de classe $C^{k}$ se todo $p \in M$ possui uma vizinhança aberta $U \subset M$ tal que $f(U) \subset N$ é aberto e $\left.f\right|_{U}: U \rightarrow f(U)$ é um difeomorfismo de classe $C^{k}$.

Segue que as cartas locais de uma variedade $M$ são nada mais que difeomorfismos entre abertos de $M$ e abertos do espaço Euclidiano. Pois, se $\left(M^{n}, \mathcal{A}\right)$ é uma variedade 
diferenciável de classe $C^{k}, U \subset M$ é um subconjunto e $W \subset \mathbb{R}^{n}$ é um aberto, então uma bijeção $\varphi: U \rightarrow W$ pertence ao atlas $\mathcal{A}$ se, e somente se, $U$ é aberto em $M$ e $\varphi$ é um difeomorfismo de classe $C^{k}$.

\subsection{Espaço Tangente}

Maioria de nós é familiar com a noção de tangente de uma circunferência ou plano tangente de uma esfera. De fato, esta noção é uma generalização dessa situação a curvas e superfícies arbitrárias ou, de forma mais geral, variedades.

Dados uma variedade diferenciável $M^{n}$ de classe $C^{k}$ e um ponto $p \in M$, denotemos por $C_{p}$ o conjunto de todas as curvas $\lambda: I \rightarrow M$ de classe $C^{k}$, com $\lambda(0)=p$, onde $I \subset \mathbb{R}$ é um intervalo aberto contendo a origem. Dizemos que duas curvas $\lambda, \mu \in C_{p}$ são equivalentes e escreveremos $\lambda \sim \mu$, se existe uma carta local $(U, \varphi)$ em $M$, com $p \in U$, tal que

$$
(\varphi \circ \lambda)^{\prime}(0)=(\varphi \circ \mu)^{\prime}(0) .
$$

Note que, como $\lambda$ e $\mu$ são contínuas e $U \subset M$ é aberto, temos que as compostas $\varphi \circ \lambda$ e $\varphi \circ \mu$ estão definidas numa vizinhança da origem em $\mathbb{R}$. Também, a definição dada em (1.1) independe da escolha da carta. Além disso, é fácil ver que a relação em $C_{p}$, definida em (1.1), é uma relação de equivalência em $C_{p}$. Dada uma curva $\lambda$ em $C_{p}$, denotaremos por $[\lambda]$ a sua classe de equivalência.

Definição 1.2.1. O espaço tangente a $M$ no ponto $p \in M$, denotado por $T_{p} M$, é definido por $T_{p} M=C_{p} / \sim$.

Dados um ponto $p \in M$ e uma carta local $(U, \varphi)$ em $M, \operatorname{com} p \in U$, definimos uma aplicação

$$
\bar{\varphi}: T_{p} M \longrightarrow \mathbb{R}^{n}, \quad \bar{\varphi}([\lambda])=(\varphi \circ \lambda)^{\prime}(0),
$$

para toda classe $[\lambda] \in T_{p} M$. Na verdade, $\bar{\varphi}$ induz uma estrutura de espaço vetorial em $T_{p} M$. De fato, dados $[\lambda],[\mu] \in T_{p} M$ temos:

$$
\bar{\varphi}([\lambda])=\bar{\varphi}([\mu]) \Longleftrightarrow(\varphi \circ \lambda)^{\prime}(0)=(\varphi \circ \mu)^{\prime}(0) \Longleftrightarrow \lambda \sim \mu \Longleftrightarrow[\lambda]=[\mu],
$$

ou seja, $\bar{\varphi}$ é injetora. Além disso, dado $v \in \mathbb{R}^{n}$, considere a curva $\alpha: I \rightarrow \varphi(U)$ definida por $\alpha(t)=\varphi(p)+t v$. Pondo $\lambda=\varphi^{-1} \circ \alpha$, temos:

$$
\bar{\varphi}([\lambda])=(\varphi \circ \lambda)^{\prime}(0)=\left(\varphi \circ \varphi^{-1} \circ \alpha\right)^{\prime}(0)=\alpha^{\prime}(0)=v,
$$

ou seja, $\bar{\varphi}$ é sobrejetora. Daí segue que

$$
\begin{aligned}
{[\lambda]+[\mu] } & =(\bar{\varphi})^{-1}(\bar{\varphi}([\lambda])+\bar{\varphi}([\mu])) \\
c \cdot[\lambda] & =(\bar{\varphi})^{-1}(c \cdot \bar{\varphi}([\lambda])) .
\end{aligned}
$$


Observe que a estrutura de espaço vetorial assim induzida em $T_{p} M$ independe da escolha da carta local e, portanto, quaisquer duas cartas locais em $M$ induzem a mesma estrutura de espaço vetorial nele.

Sejam $M^{m}, N^{n}$ duas variedades diferenciáveis de classe $C^{k}$ e $f: M \rightarrow N$ uma aplicação de classe $C^{r}, 1 \leq r \leq k$. Dado um ponto $p \in M$, definimos a diferencial $d f$ de $f$ em $p$ como uma aplicação

$$
d f_{p}: T_{p} M \longrightarrow T_{f(p)} N, \quad d f_{p}([\lambda])=[f \circ \lambda],
$$

para todo $[\lambda] \in T_{p} M$.

Dada uma carta local $(U, \varphi)$ em $M^{n}$ e $p \in U$, denotemos por

$$
\left\{\frac{\partial}{\partial x_{1}}(p), \frac{\partial}{\partial x_{2}}(p), \ldots, \frac{\partial}{\partial x_{m}}(p)\right\}
$$

a base de $T_{p} M$, induzida naturalmente pelo isomorfismo $\bar{\varphi}: T_{p} M \rightarrow \mathbb{R}^{n}$. Ou seja,

$$
\frac{\partial}{\partial x_{i}}(p)=(\bar{\varphi})^{-1}\left(e_{i}\right)
$$

para todo $1 \leq i \leq n$, onde $\left\{e_{1}, \ldots, e_{n}\right\}$ denota a base canônica de $\mathbb{R}^{n}$. Assim,

$$
\frac{\partial}{\partial x_{i}}(p)=\left[\lambda_{i}\right]
$$

onde $\lambda_{i}=\varphi^{-1} \circ \alpha_{i}$ e $\alpha_{i}: I \rightarrow \varphi(U)$ é uma curva de classe $C^{k}$ tal que $\alpha_{i}(0)=\varphi(p)$ e $\alpha_{i}^{\prime}(0)=e_{i}$, para todo $1 \leq i \leq n$.

Em particular, quando $f: M^{m} \rightarrow N^{n}$ é um difeomorfismo de classe $C^{k}$ então a sua diferencial $d f_{p}: T_{p} M \rightarrow T_{f(p)} N$ é um isomorfismo linear tal que $\left(d f_{p}\right)^{-1}=\left(d f^{-1}\right)_{f(p)}$.

Queremos também mencionar o o fibrado tangente de uma variedade diferenciável, que é o espaço natural de se trabalhar quando se trata de questões que envolvem posição e velocidade.

Seja $M^{m}$ uma variedade diferenciável de classe $C^{k}$. A cada ponto $p \in M$, associamos o espaço tangente $T_{p} M$, que é um espaço vetorial real de dimensão $m$. Denotemos por $T M$ a união disjunta de todos os espaços tangentes a $M$. Mais precisamente, definimos:

$$
T M=\bigcup_{p \in M}\{p\} \times T_{p} M .
$$

O conjunto $T M$ é chamado o fibrado tangente de $M$. Sabe-se que $T M$ pode ser visto de maneira natural como uma variedade diferenciável. De fato, se $M^{m}$ é uma variedade diferenciável de classe $C^{k}$ então o fibrado tangente $T M$ é uma variedade diferenciável de classe $C^{k-1}$ e tem dimensão $2 m$. Não apresentaremos a prova deste resultado aqui. A projeção canônica $\pi: T M \rightarrow M$ de $T M$ sobre $M$ é definida de maneira natural: $\pi(p, v)=p$, para quaisquer $p \in M$ e $v \in T_{p} M$. 


\subsection{Campos de Vetores em Variedades}

Nesta subseção discutiremos o conceito de campo vetorial, que é uma das motivações para o estudo do fibrado tangente de uma variedade diferenciável.

Definição 1.3.1. Seja $M$ uma variedade diferenciável $C^{k}$. Um campo vetorial em $M$ é uma aplicação $X: M \rightarrow T M$ tal que o diagrama

$$
\begin{aligned}
& M \stackrel{X}{\longrightarrow} T M \\
& I d \searrow \downarrow \pi \\
& \text { M }
\end{aligned}
$$

comuta.

Um campo vetorial em $M$ é também chamado de uma seção do fibrado tangente $T M$, no sentido de que $X(p) \in T_{p} M$, para todo $p \in M$. As vezes denotaremos o vetor $X(p)$ por $X_{p}$. Observe que, se $M$ é de classe $C^{k}$, um campo vetorial $X: M \rightarrow T M$ é, no máximo, uma aplicação de classe $C^{k-1}$, pois $T M$ é uma variedade de classe $C^{k-1}$.

O conjunto de todos os campos vetoriais de classe $C^{k-1}$ de uma variedade diferenciável de classe $C^{k}$ será denotado por $X(M)$. Com as operações naturais

$$
\begin{aligned}
(X+Y)(p) & =X(p)+Y(p), \\
(c X)(p) & =c X(p),
\end{aligned}
$$

para quaisquer $X, Y \in X(M), p \in M, c \in \mathbb{R}$, o conjunto $X(M)$ torna-se um espaço vetorial real.

Dados um campo vetorial $X: M \rightarrow T M$ e uma carta local $(U, \varphi)$ em $M$, podemos escrever

$$
X(p)=\sum_{i=1}^{m} a_{i}(p) \frac{\partial}{\partial x_{i}}(p),
$$

para todo $p \in U$, onde cada $a_{i}: U \rightarrow \mathbb{R}$ é uma função no aberto $U$. Considerando a carta $\bar{\varphi}: \pi^{-1}(U) \rightarrow \varphi(U) \times \mathbb{R}^{m}$ em $T M$, associada a $\varphi$, temos:

$$
\bar{\varphi}(p, X(p))=\left(\varphi(p), a_{1}(p), \ldots, a_{m}(p)\right)
$$

para todo $p \in U$. Assim,

$$
\left(\bar{\varphi} \circ X \circ \varphi^{-1}\right)(x)=\left(x,\left(a_{1} \circ \varphi^{-1}\right)(x), \ldots,\left(a_{m} \circ \varphi^{-1}\right)(x)\right)
$$

para todo $x \in \varphi(U)$. Portanto, $X$ é de classe $C^{k-1}$ se, e somente se, as funções componentes $a_{i}$ são de classe $C^{k-1}$, para todo $1 \leq i \leq m$. 
Discutiremos agora o conceito de derivações em variedades diferenciáveis obtendo, em particular, uma nova interpretação para o espaço tangente. A partir de agora, por questão de simplicidade, assumiremos que todas as variedades envolvidas são de classe $C^{\infty}$ e iremos nos referir a uma variedade diferenciável $M$ de classe $C^{\infty}$ simplesmente por uma variedade diferenciável $M$.

Dado uma variedade diferenciável $M$, denotemos por $C^{\infty}(M, \mathbb{R})$ ou simplesmente por $C^{\infty}(M)$, o espaço vetorial real das funções $f: M \rightarrow \mathbb{R}$ de classe $C^{\infty}$.

Definição 1.3.2. Seja $M$ uma variedade diferenciável e $p \in M$. Uma derivação em $p$ é um funcional linear $D: C^{\infty}(M) \rightarrow \mathbb{R}$ que satisfaz a regra de Leibniz:

$$
D(f g)(p)=(D f) g(p)+f(p)(D g)
$$

para quaisquer $f, g \in C^{\infty}(M)$.

Exemplo 1.3.3. Sejam $M$ uma variedade diferenciável e $p \in M$. Dado um vetor $v \in T_{p} M$, definimos uma função $\bar{v}: C^{\infty}(M) \rightarrow \mathbb{R}$ por

$$
\bar{v}(f)=(f \circ \lambda)^{\prime}(0)
$$

onde $\lambda: I \rightarrow M$ é uma curva de classe $C^{\infty}$ tal que $\lambda(0)=p$ e $\lambda^{\prime}(0)=v$. É fácil provar que $\bar{v}$ é uma derivação em $p$.

Exemplo 1.3.4. Dado um ponto $p \in M^{m}$ e seja $(U, \varphi)$ uma carta em $M \operatorname{com} p \in U$. Como caso particular do exemplo anterior temos, para cada $1 \leq i \leq m$, as derivações

$$
\overline{\frac{\partial}{\partial x_{i}}}(p): C^{\infty}(M) \longrightarrow \mathbb{R}
$$

Assim, dado $f \in C^{\infty}(M)$, temos:

$$
\overline{\frac{\partial}{\partial x_{i}}}(p)(f)=(f \circ \lambda)^{\prime}(0)=\frac{\partial\left(f \circ \varphi^{-1}\right)}{\partial x_{i}}(\varphi(p)),
$$

onde $\lambda: I \rightarrow U$ é uma curva diferenciável tal que $\lambda(0)=p$ e $\lambda^{\prime}(0)=\frac{\partial}{\partial x_{i}}(p)$.

Denotaremos por $\mathfrak{D} \mathfrak{e r} \mathfrak{r}_{p}(M)$ o conjunto de todas as derivações em $p$ de uma variedade diferenciável $M$. Segue que o conjunto $\mathfrak{D e r}_{p}(M)$, munido das operações

$$
\begin{aligned}
(D+T)(f) & =D f+T f \\
(c D)(f) & =c(D f)
\end{aligned}
$$

é um espaço vetorial real para quaisquer $D, T \in \mathfrak{D e r}_{p}(M), f \in C^{\infty}(M)$ e $c \in \mathbb{R}$.

De acordo com a notação usada em Exemplo 1.3.3, temos o seguinte: 
Proposição 1.3.5. Sejam $M$ uma variedade diferenciável e $p \in M$. A aplicação

$$
\phi: T_{p} M \longrightarrow \mathfrak{D e r}_{p}(M), \quad \phi(v)=\bar{v}
$$

é um isomorfismo linear.

Da Proposição 1.3.5 obtemos que os vetores tangentes em $T_{p} M$ podem ser identificados como derivações em $p$. Essa noção de derivação pode ser globalizada, como veremos na definição seguinte.

Definição 1.3.6. Seja $M$ uma variedade diferenciável. Uma derivação em $M$ é um operador linear

$$
D: C^{\infty}(M) \longrightarrow C^{\infty}(M)
$$

tal que $D(f g)=(D f) g+f(D g)$, para quaisquer $f, g \in C^{\infty}(M)$.

Exemplo 1.3.7. Dado um campo vetorial $X \in X(M)$, definimos uma aplicação

$$
\bar{X}: C^{\infty}(M) \longrightarrow C^{\infty}(M)
$$

tal que, para cada função $f \in C^{\infty}(M)$, a função $\bar{X}(f)$ é definida pondo

$$
\bar{X}(f)(p)=d f_{p} X(p),
$$

para todo $p \in M$. Daí, seque que $\bar{X}$ é uma derivação em $M$.

Seguindo a notação do Exemplo 1.3.7 temos a seguinte:

Proposição 1.3.8. Sejam $M$ uma variedade diferenciável e $X: M \rightarrow T M$ um campo vetorial. As seguintes afirmações são equivalentes:

(i) $X \in X(M)$,

(ii) $\bar{X}(f) \in C^{\infty}(M)$, para toda $f \in C^{\infty}(M)$.

Denotemos por $\mathfrak{D e r}(M)$ o espaço vetorial real de todas as derivações em $M$. A Proposição seguinte é a versão global da Proposição 1.3.5.

Proposição 1.3.9. A aplicação $\phi: X(M) \rightarrow \mathfrak{D e r}(M)$, definida por $\phi(X)=\bar{X}$, para todo $X \in X(M)$, é um isomorfismo linear.

Em virtude da Proposição 1.3.9, identificaremos naturalmente cada campo $X \in$ $X(M)$ como uma derivação em $M$ e, para cada função $f \in C^{\infty}(M)$, denotaremos simplesmente por $X(f)$ a função associada.

Dados $X, Y \in X(M)$, existe um único campo $[X, Y] \in X(M)$ tal que

$$
[X, Y](f)=X(Y(f))-Y(X(f)),
$$

para toda $f \in C^{\infty}(M)$. O campo vetorial $[X, Y] \in X(M)$ é chamado colchete de Lie dos campos $X$ e $Y$ e é usualmente denotado por $[X, Y]=X Y-Y X$. O colchete de Lie satisfaz as seguintes propriedades: 
1. $[X, Y]=-[Y, X]$

2. $[X,[Y, Z]]+[Y,[Z, X]]+[Z,[X, Y]]=0$ ( Identidade de Jacobi $)$

3. $[f X, g Y]=f g[X, Y]+f(X(g)) Y-g(Y(f)) X$, para quaisquer $X, Y, Z \in X(M)$ e $f, g \in C^{\infty}(M)$.

Observação 1.3.10. Dada uma carta local $(U, \varphi)$ em $M^{m}$, temos os campos coordenados

$$
\left\{\frac{\partial}{\partial x_{1}}, \ldots, \frac{\partial}{\partial x_{m}}\right\}
$$

asssociados a $\varphi$, ou seja, para cada $p \in U$, os vetores

$$
\left\{\frac{\partial}{\partial x_{1}}(p), \frac{\partial}{\partial x_{2}}(p), \ldots, \frac{\partial}{\partial x_{m}}(p)\right\}
$$

formam uma base para $T_{p} M$. Assim, dados $X, Y \in X(M)$, podemos representá-los, localmente, como

$$
\left.X\right|_{U}=\sum_{i=1}^{m} X_{i} \frac{\partial}{\partial x_{i}} \quad \text { e }\left.\quad Y\right|_{U}=\sum_{i=1}^{m} Y_{i} \frac{\partial}{\partial x_{i}} .
$$

Obtemos, então, a fórmula local para o colchete de $X$ e $Y$ no aberto $U$ :

$$
[X, Y]=\sum_{i, j=1}^{m}\left(X_{j} \frac{\partial Y_{i}}{\partial x_{j}}-Y_{j} \frac{\partial X_{i}}{\partial x_{j}}\right) \frac{\partial}{\partial x_{i}} .
$$

Em particular, o colchete de Lie dos campos coordenados $\frac{\partial}{\partial x_{i}}, 1 \leq i \leq m$, associados a uma carta local $(U, \varphi)$ em uma variedade diferenciável $M^{n}$ é nulo, isto é, $\left[\frac{\partial}{\partial x_{i}}, \frac{\partial}{\partial x_{j}}\right]=$ 0 , para quaisquer $1 \leq i, j \leq m$.

Exemplo 1.3.11. Um exemplo interessante da vida real referente a colchete de Lie é dado no Exemplo 1.5.6 onde os campos envolvidos são movimento linear e rotação de um carro. O colchete de Lie desses campos gera movimento na direção perpendicular à orientação do carro, e que é a manobra típica usada no dia-a-dia para estacionamento de carros.

\subsection{Curvas integrais e o Fluxo Local}

Nesta subseção faremos um estudo mais detalhado dos campos vetoriais. Mais precisamente, veremos que um campo vetorial em uma variedade diferenciável pode ser interpretado como uma equação diferencial, no sentido que passaremos a descrever. 
Definição 1.4.1. Sejam $M$ uma variedade diferenciável e $X \in X(M)$. Uma curva diferenciável $\alpha: I \rightarrow M$ é chamada curva integral de $X$ se $\alpha^{\prime}(t)=X(\alpha(t))$, para todo $t \in I$.

Dado uma carta local $(U, \varphi)$ em $M$, escrevamos

$$
X(p)=\sum_{i=1}^{m} a_{i}(p) \frac{\partial}{\partial x_{i}}(p),
$$

para todo $p \in U$. Assim, se $\alpha: I \rightarrow M$ é uma curva integral de $X$, com $\alpha(I) \subset U$, temos:

$$
\begin{aligned}
\alpha^{\prime}(t) & =X(\alpha(t)) \Longleftrightarrow(d \varphi)_{\alpha(t)} \alpha^{\prime}(t)=(d \varphi)_{\alpha(t)} X(\alpha(t)) \\
& \Longleftrightarrow \frac{d}{d t}(\varphi \circ \alpha)(t)=\sum_{i=1}^{m}\left(a_{i} \circ \alpha\right)(t) e_{i} .
\end{aligned}
$$

Assim, a condição $\alpha^{\prime}(t)=X(\alpha(t))$, para todo $t \in I$, dá a expressão local

$$
\frac{d}{d t}\left(\varphi_{i} \circ \alpha\right)=a_{i} \circ \alpha
$$

para todo $1 \leq i \leq m$, que constitui um sistema de equações diferenciais ordinárias de primeira ordem.

O teorema fundamental de existência e unicidade para as soluções de tais sistemas tem a seguinte consequência neste contexto:

Teorema 1.4.2. Sejam $M$ uma variedade diferenciável e $X \in X(M)$. Então, para cada $p \in M$, existe um intervalo aberto $I=(a, b)$ contendo 0 onde está definida a única curva integral $\alpha: I \rightarrow M$ de $X$ tal que $\alpha(0)=p$.

Uma consequência do Teorema 1.4.2 é o seguinte corolário.

Corolário 1.4.3. Sejam $\alpha_{1}: I \rightarrow M e \alpha_{2}: I \rightarrow M$ curvas integrais de um campo $X \in X(M)$ tais que $\alpha_{1}(c)=\alpha_{2}(c)$, para algum $c \in I_{1} \cap I_{2}$. Então, $\alpha_{1}(t)=\alpha_{2}(t)$, para todo $t \in I_{1} \cap I_{2}$.

Definição 1.4.4. Sejam $M$ uma variedade diferenciável e $X \in X(M)$. Um fluxo local para o campo $X$ em torno de um ponto $q \in M$ é uma aplicação $\varphi:(-\epsilon, \epsilon) \times U \rightarrow M$ de classe $C^{\infty}$, onde $U \subset M$ é um aberto contento $q$, que satisfaz as seguintes propriedades:

(i) Para cada $p \in U$, a curva $\lambda_{p}:(-\epsilon, \epsilon) \rightarrow M$, dada por $\lambda_{p}(t)=\varphi(t, p)$, é uma curva integral de $X$, com $\lambda_{p}(0)=p$.

(ii) Para cada $t \in(-\epsilon, \epsilon)$, a aplicação $\varphi_{t}: U \rightarrow M$, dada por $\varphi_{t}(p)=\varphi(t, p)$ é um difeomorfismo sobre a sua imagem. 
Posteriormente usaremos, de preferência, a notação $X_{t}$ em vez de $\varphi_{t}$ para denotar a curva integral (resp. fluxo, trajetória, etc) de um campo $X$. Seja $\varphi:(-\epsilon, \epsilon) \times U \rightarrow M$ um fluxo local para $X$. Dado $p \in U$, as curvas

$$
\lambda_{1}(t)=X_{t+s}(p) \quad \text { e } \quad \lambda_{2}(t)=X_{t}\left(X_{s}(p)\right)
$$

são curvas integrais de $X$, com $\lambda_{1}(0)=\lambda_{2}(0)=X_{s}(p)$. Assim, pelo Corolário 1.4.3, temos que:

$$
X_{t+s}(p)=X_{t}\left(X_{s}(p)\right)
$$

desde que ambos os lados estejam definidos. Disso também decorre que

$$
X_{s} \circ X_{t}=X_{t+s}=X_{t} \circ X_{s}
$$

quando definidas. Esta é a chamada propriedade local de grupo, pois $X_{t}$ se estivesse definida para todo $t \in \mathbb{R}$, então

$$
t \in \mathbb{R} \longrightarrow X_{t} \in \operatorname{Dif}(M)
$$

seria um homomorfismo de grupos.

O teorema seguinte nos assegura a existência do fluxo local.

Teorema 1.4.5. Sejam $M$ uma variedade diferenciável e $X \in X(M)$. Dado um ponto $q \in M$, existe um fluxo local $\varphi:(-\epsilon, \epsilon) \times U \rightarrow M$ para $X$ em torno de q tal que, para cada $p \in U$, a curva $\lambda_{p}:(-\epsilon, \epsilon) \rightarrow M$, dada por $\lambda_{p}(t)=\varphi(t, p)$, é a única curva integral de $X, \operatorname{com} \varphi(0, p)=p$.

Sejam $M$ uma variedade diferenciável e $X \in X(M)$. Dado um ponto $p \in M$, considere a família $\left\{\alpha_{i}: i \in I\right\}$ formada por todas as curvas integrais $\alpha_{i}:\left(-\epsilon_{i}, \epsilon_{i}\right) \rightarrow M$ de $X$, com $\alpha_{i}(0)=p$, para todo $i \in I$. O conjunto $I_{p}=\cup_{i \in I}\left(-\epsilon_{i}, \epsilon_{i}\right)$ é um intervalo aberto de $\mathbb{R}$ contendo 0. Defina uma curva $\alpha_{p}: I_{p} \rightarrow M$ pondo $\alpha_{p}(t)=\alpha_{i}(t)$, se $t \in\left(-\epsilon_{i}, \epsilon_{i}\right)$. Pelo Corolário 1.4.3, $\alpha_{p}$ está bem definida e é uma curva integral de $X$, com $\alpha_{p}(0)=p$, chamada a curva integral maximal de $X$ passando pelo ponto $p$.

Exemplo 1.4.6. Em $\mathbb{R}^{2}$, considere o campo $X=x \frac{\partial}{\partial x}-y \frac{\partial}{\partial y}$. Então, $\alpha(t)=(x(t), y(t))$ é uma curva integral de $X$ se, e somente, se

$$
\frac{d x}{d t}=x \quad \text { e } \quad \frac{d y}{d t}=-y
$$

Assim, devemos ter $x(t)=a e^{t}$ e $y(t)=b e^{-t}$, com $a, b \in \mathbb{R}$. Portanto, a curva integral maximal de $X$, passando pelo ponto $p=\left(p_{1}, p_{2}\right)$, é dada por

$$
\alpha_{p}(t)=\left(p_{1} e^{t}, p_{2} e^{-t}\right),
$$

para todo $t \in \mathbb{R}$. 
Definição 1.4.7. Um campo vetorial $X \in X(M)$ é dito ser completo se, para todo $p \in M$, o domínio da curva integral maximal de $X$ passando por $p$ é todo $\mathbb{R}$.

Dado um campo vetorial $X \in X(M)$, definimos $\mathcal{D}=\left\{(t, p): t \in I_{p}\right\}$, onde $I_{p}$ é o domínio da curva integral maximal $\alpha_{p}$ de $X$ passando por $p$. Daí pode-se definir uma aplicação diferenciável

$$
\varphi: \mathcal{D} \longrightarrow M, \quad \varphi(t, p)=\alpha_{p}(t)
$$

para todo $(t, p) \in \mathcal{D}$. A aplicação $\varphi: \mathcal{D} \rightarrow M$ é chamada o fluxo maximal do campo $X$. Observe que $X$ é completo se, e somente, se $\mathcal{D}=\mathbb{R} \times M$.

Seja $\varphi: \mathcal{D} \rightarrow M$ o fluxo maximal de um campo vetorial $X \in X(M)$. Para cada $t \in I$, defina $\operatorname{dom} X_{t}=\left\{p \in M: t \in I_{p}\right\}$ e considere a aplicação

$$
X_{t}: \operatorname{dom} X_{t} \rightarrow X_{t}\left(\operatorname{dom} X_{t}\right) \subset M
$$

definida por $X_{t}(p)=\varphi(t, p)$. Note que, em geral, o domínio de $X_{t}$ depende de $t$. Como $I_{p} \neq \varnothing$, para todo $p \in M$, segue que $M=\cup_{t>0} \operatorname{dom} X_{t}$.

Teorema 1.4.8. Dado $s \in I$, seja $t \in I$ tal que $t \in I_{\alpha_{p}(s)}$, para todo $p \in \operatorname{dom} X_{s}$. Então $t+s \in I_{p}$, para todo $p \in \operatorname{dom} X_{s}$, e vale:

$$
\left(X_{t} \circ X_{s}\right)(p)=X_{s+t}(p)
$$

para todo $p \in \operatorname{dom} X_{s}$. Decorre, em particular, que $X_{t} \circ X_{-t}=\mathrm{id}$, logo $X_{t}$ é um difeomorfismo sobre $\operatorname{dom} X_{-t}$, cujo inverso é $\left(X_{t}\right)^{-1}=X_{-t}$.

Demonstração: Veja a prova de Teorema 3.4.14 em [16].

No caso em que $X \in X(M)$ é completo, as aplicações $X_{t}$ formam um grupo de difeomorfismos de $M$ parametrizados pelos números reais, e é chamado grupo a 1-parâmetro de $X$. Se $X$ não é completo, os difeomorfismos $X_{t}$ não formam um grupo, pois seus domínios dependem de $t$. Neste caso, dizemos que a coleção dos difeomorfismos $X_{t}$ é um grupo local a 1-parâmetro de $X$.

Em suma, todo campo vetorial completo $X \in X(M)$ determina um grupo a 1parâmetro $\left\{X_{t}\right\}_{t \in \mathbb{R}}$. Reciprocamente, dado um grupo a 1-parâmetro $\left\{X_{t}\right\}_{t \in \mathbb{R}}$ de difeomorfismos de uma variedade diferenciável $M$, definimos uma aplicação $X: M \rightarrow T M$ pondo

$$
X(p)=\left(\frac{d}{d t}\right)_{t=0} X_{t}(p),
$$

para todo $p \in M$. Isso define um campo $X \in X(M)$, que tem $\left\{X_{t}\right\}_{t \in \mathbb{R}}$ como grupo a 1-parâmetro associado. 
Observação 1.4.9. É útil imaginarmos um campo vetorial $X \in X(M)$ como um campo de vetores velocidades de um fluido em movimento em $M$. Daí, o fluxo $X_{t}$ toma uma partícula desse fluido a partir de uma posição $p \in M$ e leva em tempo $t \in \mathbb{R}$ a uma posição $X_{t}(p) \in M$.

Definição 1.4.10. Seja $f: M \rightarrow N$ uma aplicação diferenciável. Os campos de vetores $X$ em $M$ e $Y$ em $N$ são ditos $f$-relacionados se df aplica $X$ em $Y$, isto é, se $d f_{x} X(x)=Y(f(x))$, para todo $x \in M$. Nesse caso a imagem por $f$ de uma curva integral, ou trajetória, de $X$ é uma trajetória de $Y$. Em termos de fluxos isso significa que $X_{t} \circ f=f \circ Y_{t}$.

Observe que dado um campo vetorial $X$ em $M$ nem sempre existe um campo em $N$ que é $f$-relacionado com $X$. No entanto, se $f: M \rightarrow N$ é um difeomorfismo e $X$ um campo em $M$ então existe um único campo em $N$, denotado por $f^{*} X$, que é $f$-relacionado com $X$. Esse campo é definido por

$$
\left(f^{*} X\right)(y)=(d f)_{f^{-1}(y)}\left(X\left(f^{-1}(y)\right) .\right.
$$

O objetivo agora é relacionar o colchete de Lie de dois campos vetoriais com seus fluxos.

Definição 1.4.11. Sejam $X$ e $Y$ dois campos de vetores. O colchete de Lie entre eles é definido por

$$
[X, Y](x)=\left(\frac{d}{d t}\right)_{t=0} d\left(X_{-t}\right)_{X_{t}(x)} Y\left(X_{t}(x)\right)
$$

Note que se $X \in X(M)$ é um campo completo então, $X_{t}$ é um difeomorfismo de $M$,para cada $t \in \mathbb{R}$ como foi visto anteriormente. No caso em que $X$ não é completo, a aplicação $X_{t}: x \mapsto X_{t}(x)$ para cada $t \in \mathbb{R}$ fixado é um difeomorfismo local de $M$ no sentido em que o domínio $\operatorname{dom} X_{t}$ de $X_{t}$ é um aberto de $M$ e $X_{t}: \operatorname{dom} X_{t} \rightarrow X_{t}\left(\operatorname{dom} X_{t}\right)$ é um difeomorfismo.

Lema 1.4.12. Sejam $X, Y$ campos de vetores diferenciáveis em $M$. Então, $[X, Y]=0$ se, e somente, se $X_{t} \circ Y_{s}=Y_{s} \circ X_{t}$ para todo $s, t$.

A comutatividade dos fluxos, dada pelo Lema anterior, pode ser interpretado da seguinte forma: Sejam $X, Y \in X(M)$. Dado $x \in M$, para todo $t$ suficientemente pequeno, façamos:

$$
\alpha(t)=\left(Y_{-t} \circ X_{-t} \circ Y_{t} \circ X_{t}\right)(x)
$$

Assim, $\alpha(t)=x$ se, e somente, se $[X, Y](x)=0$. O colchete $[X, Y]$ mede a abertura da curva em (1.13). 
Com a equação dada em (1.12) se define derivada de Lie de um campo $Y$ com respecto a outro campo $X$ em um ponto $x \in M$. De fato, se $X$ e $Y$ são campos diferenciáveis em uma variedade diferenciável $M$ pode-se seguir primeiro o fuxo de $X$ a partir de $x$ até $X_{t}(x)$ e avaliar $Y$ nesse último instante. Daí podemos transportar de volta o vetor $Y\left(X_{t}(x)\right)$ a $T_{x} M$ através da diferencial $d X_{-t}$ do difeomorfismo $X_{-t}$ no ponto $X_{t}(x)$. Em $T_{x} M$, podemos considerar o quociente formado pela divisão da diferença $d X_{-t} Y\left(X_{t}(x)\right)-Y(x)$ por $t$ e, tomar o limite quando $t \rightarrow 0$. Em outras palavras, podemos considerar a aplicação diferenciável $t \mapsto d X_{-t} Y\left(X_{t}(x)\right)$ a valores em $T_{x} M$ e, derivar em $t=0$. O que resulta é um vetor em $T_{x} M$, que é denominado de derivada de Lie de $Y$ com respecto a $X$ em $x$ e denotado por $L_{X}(Y)(x)$. Portanto, pode-se definir:

$$
\begin{aligned}
L_{X}(Y)(x) & =\lim _{t \rightarrow 0} \frac{d\left(X_{-t}\right)_{X_{t}(x)} Y\left(X_{t}(x)\right)-Y(x)}{t} \\
& =\left(\frac{d}{d t}\right)_{t=0} d\left(X_{-t}\right)_{X_{t}(x)} Y\left(X_{t}(x)\right) .
\end{aligned}
$$

Proposição 1.4.13. Sej X um campo diferenciável em uma variedade diferenciável M. Então

$$
L_{X}(Y)=[X, Y]
$$

para todo campo diferenciável $Y$ em $M$.

\subsection{Sistemas de Controle em Variedades}

Esta pequena seção, que foi tomada de [1], tem por objetivo dar ao leitor as ideias e conceitos básicos de sistemas de controle em variedades com o intutito de melhorar o entendimento dos últimos dois capitulos.

Um sistema dinâmico diferenciável, ou uma equação diferencial ordinária (abrev. EDO), em uma variedade diferenciável $M$ é uma equação da forma

$$
\frac{d x}{d t}=X(x), \quad x \in M
$$

onde $X(x)$ é um campo diferenciável em $M$. Denotamos por $\varphi\left(t, x_{0}\right)$ a solução desse sistema. Segue então que para sistemas dinâmicos, o futuro $\varphi\left(t, x_{0}\right), t>0$, é completamente determinado pelo estado presente $x_{0}=\varphi\left(0, x_{0}\right)$.

A Lei de transformação $x_{0} \mapsto \varphi\left(t, x_{0}\right)$ é nada mais que o fluxo $\varphi_{t}$, isto é, a dinâmica do sistema (1.16) é determinada por um só campo vetorial $X(x)$.

Para poder influenciar a dinâmica ou controlá-la considera-se uma família de sistemas dinâmicos

$$
\dot{x}=X(x, u)=X_{u}(x), \quad x \in M, \quad u \in U,
$$


com a família de campos $X_{u}$ parametrizados por um parâmetro $u \in U$.

Definição 1.5.1. Um sistema da forma (1.17) é chamada um sistema de controle.

Segue da definição que a dinâmica de um sistema de controle é dada por uma família de equações diferenciais que dependem da variável u chamada parâmetro de controle ou simplesmente controle. Portanto, a teoria de sistemas de controle pode ser vista como uma teoria de famílias de campos de vetores (parametrizados) ou polisistemas dinâmicos. O conjunto $U$ é o espaço de controles. A princípio, nenhuma restrição é imposta sobre $U$. Pode ser um conjunto arbitrário ou um subconjunto de uma variedade diferenciável. Mas geralmente algumas restrições são necessárias para que a equação (1.17) faça sentido. Então é natural que $U$ contenha controles admissíveis. O pre-requisito mínimo para que um controle $u$ seja admissível é que seja, por exemplo, localmente integrável sem qual a equação diferencial em (1.17) não teria sentido. Por outro lado, como restringimos a nossa atenção à natureza do problema tratado o conjunto $U$ pode alocar também controles mensuráveis e limitadas, controles constantes por pedaços ou até mesmo controles extremos que assumem seus valores apenas nos vértices de um cubo unitário (i.e., controles bang-bang), etc.

Em geral, basta nos preocuparmos em controles constantes por pedaços. Por um controle $u$ constante por pedaços entendemos o seguinte: O domínio de tempo de $u$ se decompõe em subintervalos pequenos cujos comprimentos são inferiormente limitados de tal forma que $u$ é constante em cada um desses subintervalos. O conjunto desses controles é denotado por $U_{p c}$. As soluções do sistema (1.17) com um controle proveniente de $U_{p c}$ são chamadas trajetórias constantes por pedaços.

Quanto à variável $x$, a chamamos de estado e, por conseguinte, a variedade $M$ torna-se o espaço estado do sistema de controle (1.17). Asumimos que para todo $u \in U$ a aplicação $X_{u}: p \mapsto X_{u}(p)$ de $M$ no fibrado tangente $T M$ de $M$ é um campo de vetores diferenciável em $M$. Isto garante que para todo $u \in U$ e $x \in M$ a equação diferencial (1.17) tem soluções locais maximais únicas $\operatorname{com} \varphi\left(0, x_{0}, u\right)=x_{0}$ no tempo $t=0$.

Na Teoria do controle podemos alterar dinâmicas do sistema de controle (1.17) em qualquer momento trocando valores de $u \in U$. Dado um $u \in U$, denotamos por $X_{t}^{u}$ ou simplesmente por $X_{t}$ o fluxo gerado pelo campo vetorial $X_{u} \in X(M)$ por $u$ parametrizado.

Um problema típico da teoria é encontrar o conjunto de pontos (estados) que podem ser alcançados a partir de um ponto inicial $x_{0} \in M$ escolhendo diversos valores de $u \in U$ e fazendo de vez em quando trocas de um valor para outro. Note que para um sistema dinâmico (1.16) o conjunto de pontos acessíveis é apenas a semitrajetória $\varphi\left(t, x_{0}\right)=X_{t}\left(x_{0}\right), t>0$. 
Agora, suponha que partimos de um ponto $x_{0} \in M$ e que usamos a seguinte estratégia de controle para o sistema (1.17): Escolha primeiro algum parâmetro de controle $u_{1} \in U$ para começar e depois troque-o por um outro controle $u_{2} \in U$. E denotamos por $X_{t_{1}}^{1}$ e $X_{t_{2}}^{2}$ os fluxos dos campos $X_{u_{1}}$ e $X_{u_{2}}$ parametrizados respectivamente por $u_{1}$ e $u_{2}$.

A pergunta natural que surge a esta altura é quais pontos em $M$ podem ser acessíveis com tal estratégia de controle. Observe que com o controle $u_{1}$ podemos atingir pontos da forma

$$
\left\{X_{t_{1}}^{1}\left(x_{0}\right) \in M: t_{1} \geq 0\right\}
$$

enquanto que o conjunto inteiro de pontos acessíveis tem a forma

$$
\left\{X_{t_{2}}^{2} \circ X_{t_{1}}^{1}\left(x_{0}\right) \in M: t_{1}, t_{2} \geq 0\right\}
$$

que é porção de uma superfície de dimensão 2.

A próxima pergunta seria quais pontos em $M$ podem ser alcançados a partir de $x_{0} \in M$ por qualquer tipo estratégia de controle.

Achamos conveniente considerar aqui apenas um caso particular de um sistema de controle que dá modelamento simplificado de um carro. Este mesmo exemplo além de [1] pode ser encontrado também em [17]

Exemplo 1.5.2. Suponha que o estado de um carro seja determinado pela posição de seu centro de massa $x=\left(x_{1}, x_{2}\right) \in \mathbb{R}^{2}$ e o ângulo de orientação $\theta \in S^{1}$ relativo a direção positiva do eixo- $x_{1}$. Portanto, o espaço estado de nosso sistema é a variedade tri-dimensional

$$
M=\left\{p=(x, \theta): x \in \mathbb{R}^{2}, \theta \in S^{1}\right\}=\mathbb{R}^{2} \times S^{1},
$$

tôro sólido. Suponha que apenas dois tipos de movimentos sejam possíveis, a ssber, pode-se dirigir para a frente ou para trás com alguma velocidade linear fixada $u_{1} \in \mathbb{R}$, e que pode-se virar o carro ao redor de seu centro de massa com alguma velocidade angular linear fixada $u_{2} \in \mathbb{R}$. Podemos combinar de uma forma admissível as duas alternativas de movimento. De fato, o sistema dinâmico que descreve o movimento linear com a velocidade $u_{1} \in U$ tem a forma

$$
\begin{aligned}
& \dot{x_{1}}=u_{1} \cos \theta \\
& \dot{x_{2}}=u_{1} \sin \theta \\
& \dot{\theta}=0 .
\end{aligned}
$$

Rotação com velocidade angular $u_{2} \in U$ pode ser descrita como

$$
\begin{aligned}
& \dot{x_{1}}=0 \\
& \dot{x_{2}}=0 \\
& \dot{\theta}=u_{2} .
\end{aligned}
$$


O controle $u=\left(u_{1}, u_{2}\right)$ pode assumir qualquer valor no subconjunto dado $U \subset \mathbb{R}^{2}$. Se escrevermos as EDOs (1.18) e (1.19) na forma vetorial:

$$
\dot{p}=u_{1} X^{1}(p), \quad \dot{p}=u_{2} X^{2}(p),
$$

onde

$$
X^{1}(p)=\left(\begin{array}{c}
\cos \theta \\
\sin \theta \\
0
\end{array}\right), \quad X^{2}(p)=\left(\begin{array}{l}
0 \\
0 \\
1
\end{array}\right)
$$

então teremos o nosso modelo como

$$
\dot{p}=X_{u}(p)=u_{1} X^{1}(p)+u_{2} X^{2}(p), \quad p \in M, \quad u \in U
$$

Retornando ao estudo de pontos acessíveis por trajetórias de um sistema de controle a partir de um ponto inicial, apresentamos a seguinte

Definição 1.5.3. O conjunto acessivel de sistema de controle (1.17) com controles constantes por pedaços a partir de um ponto $x_{0} \in M$ por algum tempo $t \geq 0$ é definido como:

$$
\mathcal{A}_{\Sigma}\left(x_{0}, t\right)=\left\{X_{t_{k}}^{k} \circ \cdots \circ X_{t_{1}}^{1}\left(x_{0}\right): t_{k} \geq 0, \sum_{i=1}^{k} t_{k}=t, u_{i} \in U, k \in \mathbb{N}\right\} .
$$

O conjunto acessivel a partir de $x_{0}$ por um tempo arbitrário não-negativo tem a forma

$$
\mathcal{A}_{\Sigma}\left(x_{0}\right)=\bigcup_{t \geq 0} \mathcal{A}_{\Sigma}\left(x_{0}, t\right)
$$

Por questão de simplicidade, considere primeiro o menor espaço não-trivial de controles que contém dois índices: $U=\{1,2\}$. O conjunto acessível por um tempo arbitrário não-negativo é da forma:

$$
\mathcal{A}_{\Sigma}\left(x_{0}\right)=\left\{X_{t_{k}}^{2} \circ X_{t_{k=1}}^{1} \circ \cdots \circ X_{t_{2}}^{2} \circ X_{t_{1}}^{1}\left(x_{0}\right): t_{k} \geq 0, k \in \mathbb{N}\right\}
$$

Esta expressão sugere que o conjunto acessível $\mathcal{A}_{\Sigma}\left(x_{0}\right)$ depende fortemente de comutatividade de fluxos $X_{t}^{1}$ e $X_{s}^{2}$. Com efeito, suponha que os fluxos comutam, o que significa por sua vez que

$$
X_{t}^{1} \circ X_{s}^{2}=X_{s}^{2} \circ X_{t}^{1}
$$

para todo $s, t \in \mathbb{R}$. Daí, o conjunto acessível pode ser determinado precisamente pois

$$
X_{t_{k}}^{2} \circ X_{t_{k=1}}^{1} \circ \cdots \circ X_{t_{2}}^{2} \circ X_{t_{1}}^{1}=X_{t_{2}+\cdots+t_{k}}^{2} \circ X_{t_{1}+\cdots+t_{k-1}}^{1}
$$

e, portanto,

$$
\mathcal{A}_{\Sigma}\left(x_{0}\right)=\left\{X_{s}^{2} \circ X_{t}^{1}: t, s \geq 0\right\} .
$$


Então, no caso comutativo o conjunto acessível por dois controles é porção de uma superfície suave de dimensão 2, possivelmente com singularidades. Quando o número de controles $k \geq 2$ e os fluxos correspondentes $X_{t_{1}}^{1}, \ldots, X_{t_{k}}^{k}$ comutam então $\mathcal{A}_{\Sigma}\left(x_{0}\right)$ é, em geral, porção de uma variedade $k$-dimensional e, em particular, $\operatorname{dim} \mathcal{A}_{\Sigma}\left(x_{0}\right) \leq k$.

Note que o caso comutativo é muito excepcional e que quase nunca ocorre em sistemas de controle reais.

Exemplo 1.5.4. No exemplo de carro visto anteriormente, a dinâmica do controle é definida por dois campos vetoriais $X^{1}$ e $X^{2}$ de (1.20) na variedade tri-dimensional $M=\mathbb{R}^{2} \times S^{1}$. Dada uma configuração inicial $p_{0}=\left(x_{0}, \theta_{0}\right) \in M$ pode-se dirigir a qualquer configuração terminal $p_{1}=\left(x_{1}, \theta_{1}\right) \in M$ alternando movimentos lineares e rotações (ambos com velocidades fixadas). Daí resulta que todo ponto em $M$ pode ser alcançado através dos campos $X^{1}$ e $X^{2}$. Isto porque seus fluxos não comutam.

Observação 1.5.5. No caso comutativo, o conjunto acessível não depende de número de trocas que uma estratégia preve enquanto que no caso geral de não-comutatividade, ao contrário, maior número de trocas forcenem mais pontos alcançados.

Exemplo 1.5.6. Lembramos que para dois campos $X^{1}$ e $X^{2}$ em $\mathbb{R}^{n}$ o seu colchete é dado como

$$
\left[X^{1}, X^{2}\right](x)=\frac{d X^{2}}{d x} X^{1}(x)-\frac{d X^{1}}{d x} X^{2}(x) .
$$

No exemplo de carro, o campo $X^{1}$ gera movimento para a frente e $X^{2}$ a rotação do carro no sentido anti-horário. Então

$$
\begin{aligned}
{\left[X^{1}, X^{2}\right](p) } & =\frac{d X^{2}}{d p} X^{1}(p)-\frac{d X^{1}}{d p} X^{2}(p) \\
& =\left(\begin{array}{ccc}
0 & 0 & -\sin \theta \\
0 & 0 & \cos \theta \\
0 & 0 & 0
\end{array}\right)\left(\begin{array}{l}
0 \\
0 \\
1
\end{array}\right) \\
& =\left(\begin{array}{c}
\sin \theta \\
-\cos \theta \\
0
\end{array}\right) .
\end{aligned}
$$

O campo $\left[X^{1}, X^{2}\right]$ gera movimento do carro na direção perpendicular à orientação do carro. Esta é, na verdade, a típica manobra de estacionamento de um carro, ou seja, uma sequência de 4 movimentos com a mesma pequena amplitude da forma, a saber, (i) movimento para a frente, (ii) rotação sentido anti-horário, (iii) movimento para trás e (iv) rotação sentido horário. Tal sequência resulta movimento do carro para a direita em termos de posicionamento. 



$\frac{1}{2}$

\section{Grupos de Lie e Suas Álgebras de Lie}

Os conceitos de grupos de Lie e suas álgebras de Lie que iremos apresentar neste capítulo foram tomadas em grande parte das notas não publicadas de San Martin [22] sobre Grupos de Lie e também o livro de álgebras de Lie do mesmo autor, [23]. Este capítulo será útil para melhor compreendermos depois a introdução de sistemas lineares em grupos de Lie.

Os grupos de Lie formam uma das classes mais importantes de variedades diferenciáveis. De fato, eles são variedades diferenciáveis que também são grupos no qual as operações de grupo são diferenciáveis. A álgebra de Lie $\mathfrak{g}$ de um grupo de Lie $G$ é definida como o espaço dos campos invariantes (à esquerda ou à direita), com o colchete dado pelo colchete de Lie de campos de vetores. Os fluxos dos campos invariantes estabelecem a aplicação exponencial exp : $\mathfrak{g} \rightarrow G$, que é o principal mecanismo de ligação entre $\mathfrak{g}$ e $G$.

\subsection{Grupos de Lie}

Definição 2.1.1. (Grupo de Lie) Um grupo de Lie é um grupo cujo conjunto subjacente tem uma estrutura de variedade diferenciável, de tal forma que a aplicação produto

$$
p: G \times G \longrightarrow G, \quad(g, h) \longmapsto g h,
$$

é diferenciável. 
Tanto a estrutura de variedade diferenciável de $G$, quanto a diferenciablidade de $p$, pressupõem um grau de diferenciabilidade $C^{k}, 1 \leq k \leq \omega$. Porém, será assumido que $G$ é de classe $C^{\infty}$ assim como o produto $p$.

Exemplos 2.1.2. 1. Qualquer espaço vetorial real $V$ de dimensão finita é um grupo de Lie abeliano, com a operação de adição + em $V$.

2. Seja $\operatorname{Gl}(n, \mathbb{R})$ o grupo das transformações lineares inversíveis de $\mathbb{R}^{n}$, ou o que é a mesma coisa, o grupo das matrizes $n \times n$ inversíveis com etradas reais. Esse grupo é um subconjunto aberto do espaço vetorial $\mathrm{M}_{n}(\mathbb{R})$ das matrizes $n \times n$, e portanto é uma variadede diferenciável. O produto no grupo $\operatorname{Gl}(n, \mathbb{R})$ é proveniente do produto usual de matrizes. Se $A=\left(a_{i j}\right)$ e $B=\left(b_{i j}\right)$ são matrizes $n \times n$, então $C=A B=\left(c_{i j}\right)$ é dado por $c_{i j}=\sum_{k=1}^{n} a_{i k} b_{k j}$, que é um polinômio de grau dois nas variáveis $a_{i j}, b_{i j}$ e, portanto, é uma aplicação diferenciável. Por essa razão $\operatorname{Gl}(n, \mathbb{R})$ é um grupo de Lie. No caso em que tem-se um espaço vetorial real $V$ de dimensão finita, denota-se por $\operatorname{Gl}(V)$ o grupo das transformações lineares inversíveis de $V$.

3. (Grupo de Heisenberg de dimensão 3) Seja $G$ o grupo das matrizes da forma

$$
\left(\begin{array}{lll}
1 & x & y \\
0 & 1 & z \\
0 & 0 & 1
\end{array}\right), \quad x, y, z \in \mathbb{R}
$$

A aplicação $G \rightarrow \mathbb{R}^{3}$ que associa a uma matriz em (2.1) uma terna $(x, y, z)$ é um difeomorfismo de $G$ sobre $\mathbb{R}^{3}$ e, portanto, a operação binária em $G$ é dada pela multiplicação usual de matrizes, ou seja,

$$
\left(x_{1}, x_{2}, x_{3}\right) \cdot\left(y_{1}, y_{2}, y_{3}\right)=\left(x_{1}+y_{1}, x_{2}+y_{2}, x_{3}+y_{3}+x_{1} y_{2}\right)
$$

que por sua vez é diferente da adição vetorial em $\mathbb{R}^{3}$. Eis porque a referida correspondência deixa de ser um isomorfismo algébrico.

Dado $g \in G$, as translações à esquerda e à direita $L_{g}: G \rightarrow G$ e $R_{g}: G \rightarrow G$, são definidas respectivamente por $L_{g}(h)=g h$ e $R_{g}(h)=h g$. Essas aplicações são diferenciáveis pois $L_{g}=p \circ s_{g, 1}$ e $R_{g}=p \circ s_{g, 2}$ onde $s_{g, 1}(h)=(g, h)$ e $s_{g, 2}(h)=(h, g)$ são aplicações diferenciáveis $G \rightarrow G \times G$. Na verdade, ambas as translações, à esquerda e à direita, são difeomorfismos, já que $L_{g} \circ L_{g^{-1}}=R_{g} \circ R_{g^{-1}}=$ id. Da mesma forma, os automorfismos internos (ou conjugações) $C_{g}=L_{g} \circ R_{g^{-1}}, g \in G$, são difeomorfismos.

Ao contrário dos grupos topológicos a definição de grupo de Lie não exige a priori que a inversa $\iota(g)=g^{-1}$ seja diferenciável ou sequer contínua. A razão para isso é que a diferenciabilidade de $p$ implica a de $\iota$ através do teorema da função implícita. 
Proposição 2.1.3. Num grupo de Lie $G$ a aplicação $\iota: g \in G \longmapsto g^{-1} \in G$ é um difeomorfismo. A diferencial de ı é dada por

$$
d \iota_{g}=-\left(d L_{g^{-1}}\right)_{e} \circ\left(d R_{g^{-1}}\right)_{g}
$$

Em particular, $d \iota_{e}=-\mathrm{id}$.

Demonstração: Veja Proposição 3.1 em [22].

Sejam $G$ e $H$ grupos de Lie. Então, o produto cartesiano $G \times H$ admite a estrutura de variedade produto e a estrutura de grupo produto $\left(g_{1}, h_{1}\right)\left(g_{2}, h_{2}\right)=\left(g_{1} g_{2}, h_{1} h_{2}\right)$, tornando $G \times H$ um grupo de Lie. De fato, a diferenciabilidade do produto é consequência de que cada coordenada é diferenciável. De maneira mais geral, o produto direto de um número finito de grupos de Lie também é um grupo de Lie com as estruturas produto. Posteriormente, serão feitas outras construções com grupos de Lie, tais como o produto semi-direto e o quociente de um grupo por um subgrupo, etc.

Definição 2.1.4. (Subgrupo de Lie) Seja $G$ um grupo de Lie e $H \subset G$ um subgrupo. Então, $H$ é um subgrupo de Lie de $G$ se $H$ é uma subvariedade imersa de $G$ tal que o produto $H \times H$ é diferenciável em relação à estrutura intrínseca de $H$.

Seja $H \subset G$ um subgrupo de Lie. Se $h \in H$ então as translações $R_{h}$ e $L_{h}$ são difeomorfimos de $G$ que se restringem a difeomorfismos de $H$, pois $H$ também é grupo de Lie.

Exemplos 2.1.5. 1. Seja $\mathrm{O}(n)=\left\{g \in \mathrm{Gl}(n, \mathbb{R}): g^{T} g=g g^{T}=I\right\}$ o subgrupo das matrizes ortoganais $n \times n$. Para verificar que $\mathrm{O}(n)$ é um subgrupo de Lie de $\operatorname{Gl}(n, \mathbb{R})$ considere a aplicação $\tau: \operatorname{Gl}(n, \mathbb{R}) \rightarrow \operatorname{Gl}(n, \mathbb{R})$ dado por $\tau(g)=g^{T} g$, onde $g^{T}$ significa transposta de matriz $g \in \mathrm{Gl}(n, \mathbb{R})$. Segue então que $\mathrm{O}(n)=\tau^{-1}\{1\}$. Por outro lado, se $A$ é uma matriz então $d \tau_{g}(A)=A^{T} g+g^{T} A=\left(g^{T} A\right)^{T}+g^{T} A$. Daí que o núcleo de $d \tau_{g}$ é dado por

$$
\text { ker } d \tau_{g}=\left\{\left(g^{T}\right)^{-1} B: B^{T}+B=0\right\},
$$

que é a translação à esquerda por $\left(g^{T}\right)^{-1}$ do espaço das matrizes simétricas. Portanto, $\tau$ tem posto constante em todo ponto de $\operatorname{Gl}(n, \mathbb{R})$. Em particular, $\mathrm{O}(n)=\tau^{-1}\{1\}$ é uma subvariedade mergulhada de $\operatorname{Gl}(n, \mathbb{R})$, o que mostra que o grupo ortogonal é subgrupo de Lie.

Como componente conexa do elemento neutro o grupo $\mathrm{SO}(n)$ é também um subgrupo de Lie. Em particular, tem-se o grupo $\mathrm{SO}(3)$

$$
\mathrm{SO}(3)=\left\{g \in \mathrm{Gl}(3, \mathbb{R}): \operatorname{det} g=1 \text { e } g^{T} g=g g^{T}=I\right\}
$$

das rotações em $\mathbb{R}^{3}$. 
2. Outros grupos de Lineares, isto é, subgrupos de Lie de $\operatorname{Gl}(n, \mathbb{R})$ são:

(i) Grupo linear especial, $\operatorname{Sl}(n, \mathbb{R})=\{g \in \mathrm{Gl}(n, \mathbb{R}): \operatorname{det} g=1\}$,

(ii) Grupo Unitário, $\mathrm{U}(n)$ das matrizes complexas $n \times n$ tais que $\bar{g}^{T} g=g \bar{g}^{T}=1$,

(iii) Grupo simplético real, $\operatorname{Sp}(n, \mathbb{R})$ formado pelas matrizes reais $n \times n$ tais que $g^{T} J g=g J g^{T}=J$ onde

$$
J=\left(\begin{array}{cc}
0 & -\mathrm{id}_{n \times n} \\
\operatorname{id}_{n \times n} & 0
\end{array}\right)
$$

Definição 2.1.6. (Homomorfismo de grupos de Lie) Sejam $G$ e H dois grupos de Lie. Um homomorfismo $\phi: G \rightarrow H$ diferenciável entre $G$ e $H$ é chamado de homomorfismo de grupos de Lie.

A mesma terminologia acima se aplica a isomorfismos e automorfismos de grupos de Lie. Ou seja, no caso em que $\phi$ possui uma inversa que também é um homomorfismo de grupos de Lie, dizemos que $\phi$ é um isomorfismo de grupos de Lie. Quando $G=H$ um isomorfismo $\phi: G \rightarrow H$ entre grupos de Lie é chamado de automorfismo de grupos de Lie. Denotaremos por $\operatorname{Aut}(G)$ o grupo dos automorfismos de $G$, que terá especial atenção nas seções subsequentes.

Na Definição 2.1.6 poderíamos supor, sem perda de generalidade, que $\phi$ fosse apenas contínuo pois todo homomorfismo contínuo entre grupos de Lie é imediatemente diferenciável. Como a condição de ser diferenciável faz parte da definição de homomorfismo de grupos de Lie, achamos conveniente comentar que diferenciabilidade de um homomorfismo $\phi: G \rightarrow H$ entre grupos de Lie se reduz a diferenciabilidade em um único ponto. De fato, valem as igualdades

$$
\phi \circ R_{g}=R_{\phi(g)} \circ \phi \quad \text { e } \quad \phi \circ L_{g}=L_{\phi(g)} \circ \phi .
$$

Da primeira delas se obtem $\phi=R_{\phi(g)} \circ \phi \circ R_{g^{-1}}$. Aplicando a regra da cadeia se vê que se é diferenciável no elemento neutro $e \in G$ então também é diferenciável em $g \in G$.

Abaixo definimo a noção de produto semi-direto de dois grupos que generaliza o produto direto.

Definição 2.1.7. (Produto semi-direto de grupos de Lie) Sejam $G$ e $H$ dois grupos de Lie e $\tau: G \rightarrow \operatorname{Aut}(H)$ um homomorfismo (diferenciável). Cada elemento $(g, h) \in$ $G \times H$ define duas aplicações afins de $H$ uma à esquerda e outra à direita, dadas por $h \tau(g)(x)$ e $\tau(g)(x) h, x \in H$. Através da composta dessas aplicações afins se obtém duas estruturas de grupos em $G \times H$, que se denomina de produto semi-direto (à esquerda ou à direia) de $G$ e $H$ por $\tau$. Os produtos são dados explicitamente por

(i) $\left(g_{1}, h_{1}\right)\left(g_{2}, h_{2}\right)=\left(g_{1} g_{2}, h_{1} \tau\left(g_{1}\right)\left(h_{2}\right)\right)$

(ii) $\left(g_{1}, h_{1}\right)\left(g_{2}, h_{2}\right)=\left(g_{1} g_{2}, \tau\left(g_{1}\right)\left(h_{2}\right) h_{1}\right)$. 
O produto semi-direto é denotado por $G \otimes H$ e como $\tau$ é um homomorfismo diferenciável, os produtos dados acima são diferenciáveis e, portanto, o produto semi-direto de grupos de Lie é grupo de Lie. A álgebra de Lie de um produto semi-direto $G \otimes H$ é dada pelo produto semi-direto de suas álgebras de Lie. Veja a Definição 2.3.10

\section{2 Álgebra de Lie de um Grupo de Lie}

O primeiro passo no estudo dos grupos de Lie consiste na construção das álgebras de Lie associadas.

Definição 2.2.1. (Álgebra de Lie) Uma álgebra de Lie consiste de um espaço vetorial $\mathfrak{g}$ munido de um produto (colchete) $[\cdot, \cdot]: \mathfrak{g} \times \mathfrak{g} \rightarrow \mathfrak{g}$ que satisfaz as seguintes propriedades:

1. é bilinear, isto é, linear em cada uma das variáveis.

2. anti-simétria, isto é, $[X, Y]=-[Y, X]$, para todo $X, Y \in \mathfrak{g}$.

3. identidade de Jacobi, isto é, para todo $X, Y, Z \in \mathfrak{g}$,

$$
[X,[Y, Z]]=[[X, Y], Z]+[Y,[X, Z]]
$$

Note que esta igualdade pode ser reescrita alternativamente da forma

$$
[[X, Y], Z]=[[X, Z], Y]+[X,[Y, Z]]
$$

Existe uma razão especial para escrever a identidade de Jacobi nesta forma; veja a seguir representações adjuntas e derivações de álgebras de Lie.

Definição 2.2.2. (Subálgebra de Lie) Seja $\mathfrak{g}$ uma álgebra de Lie. Uma subálgebra de Lie de $\mathfrak{g}$ é um subespaço vetorial $\mathfrak{h}$ de $\mathfrak{g}$ que é fechado pelo colchete, isto é, $[X, Y] \in \mathfrak{h}$ se $X, Y \in \mathfrak{h}$.

Exemplos 2.2.3. 1. Um exemplo de álgebra de Lie é dado pelo espaço vetorial dos campos de vetores sobre uma variedade diferenciável $\left(C^{\infty}\right)$ munido do colchete de Lie de campos de vetores.

2. Outro exemplo é a álgebra $\mathfrak{g l}(n, \mathbb{R})$ formada pelas matrizes reais $n \times n$ com o colchete dado pelo comutador de matrizes

$$
[A, B]=A B-B A .
$$

3.Os campos invariantes em $\left(\mathbb{R}^{n},+\right)$ são os campos constantes. Como o colchete de Lie de campos constantes se anula, a álgebra de Lie $\mathbb{R}^{n}$ do grupo de Lie abeliano $\mathbb{R}^{n}$ é abeliana, isto é, satisfaz $[\cdot, \cdot] \equiv 0$. 
4. Se $G$ e $H$ são grupos de Lie com álgebras de Lie $\mathfrak{g}$ e $\mathfrak{h}$, respectivamente então a álgebra de Lie de $G \times H$ é $\mathfrak{g} \times \mathfrak{h}$, onde o colchete pe dado por

$$
\left[\left(X_{1}, Y_{1}\right),\left(X_{2}, Y_{2}\right)\right]=\left(\left[X_{1}, X_{2}\right],\left[Y_{1}, Y_{2}\right]\right)
$$

De maneira mais geral, a álgebra de Lie de um número finito de produto direto $G_{1} \times$ $\cdots \times G_{k}$ é o produto direto $\mathfrak{g}_{1} \times \cdots \times \mathfrak{g}_{k}$ de suas álgebras de Lie, em que o colchete é dado coordanada a coordenada.

5. (Álgebra de Heisenberg de dimensão 3) Seja g a álgebra de Lie do grupo de Heisenberg visto no itém 4 de Exemplos 2.1.2. Referente aos três vetores-base do $\mathbb{R}^{3}$ pode-se definir três curvas distintas em $G$. Mais precisamente, define-se primeiro a curva $\gamma: \mathbb{R} \rightarrow \mathbb{R}^{3}$ dada por $\gamma(t)=(t, 0,0)$ que passa pela origem de $\mathbb{R}^{3}$. Agora, podemos definir uma curva em $G$ passando pela identidade $e \in G$ pondo $\phi=\varphi^{-1} \circ \gamma$ onde $\varphi: G \rightarrow \mathbb{R}^{3}$ é o difeomorfismo entre $G$ e $\mathbb{R}^{3}$ :

$$
\phi(t)=\varphi^{-1}(\gamma(t))=\left(\begin{array}{ccc}
1 & t & 0 \\
0 & 1 & 0 \\
0 & 0 & 1
\end{array}\right) \in G,
$$

para todo $t \in \mathbb{R}$. Tomando a derivada dessa curva na identidade $e$ obtem-se um dos geradores da álgebra de Heisenberg $\mathfrak{g}$. Isto é,

$$
\left(\frac{d}{d t}\right)_{t=0} \phi(t)=\left(\begin{array}{lll}
0 & 1 & 0 \\
0 & 0 & 0 \\
0 & 0 & 0
\end{array}\right)=Y^{1} \in \mathfrak{g} .
$$

Analogamente, obtemos os campos

$$
Y^{2}=\left(\begin{array}{lll}
0 & 0 & 0 \\
0 & 0 & 1 \\
0 & 0 & 0
\end{array}\right) \quad \text { e } \quad Y^{3}=\left(\begin{array}{lll}
0 & 0 & 1 \\
0 & 0 & 0 \\
0 & 0 & 0
\end{array}\right)
$$

e por conseguinte, a álgebra de Heisenberg como $\mathfrak{g}=\operatorname{Span}\left(Y^{1}, Y^{2}, Y^{3}\right\}$. Segue que $\left[Y^{1}, Y^{2}\right]=Y^{3}$ é o único colchete não-nulo. Portanto, a notação que usaremos é $\mathfrak{g}=$ $\operatorname{Lie}\left\{Y^{1}, Y^{2}\right\}$.

Observação 2.2.4. (i) A álgebra de Heisenberg de dimensão 3 também pode ser escrita como $\mathfrak{g}=\mathbb{R} Y^{1}+\mathbb{R} Y^{2}+\mathbb{R} Y^{3}$ onde $Y^{1}=\frac{\partial}{\partial x_{1}}, Y^{2}=x_{3} \frac{\partial}{\partial x_{1}}+\frac{\partial}{\partial x_{2}}$ e $Y^{3}=\frac{\partial}{\partial x_{3}}$. Daí o único colchete de Lie não-nulo é $\left[Y^{3}, Y^{2}\right]=Y^{1}$.

(ii) A álgebra de Lie $\mathfrak{g}$ de grupo de Heisenberg de dimensão $2 p+1$ é gerada pelos campos

$$
X_{1}, X_{2}, \ldots, X_{p}, Y_{1}, Y_{2}, \ldots, Y_{p}, Z
$$


com os únicos colchetes de Lie não-culo sendo

$$
\left[X_{i}, Y_{i}\right]=Z, \quad 1 \leq i \leq p .
$$

Segue que $\mathfrak{g}$ tem uma realização sobre o espaço vetorial de matrizes triangulares superiores de ordem $p+2$ com o comutador usual $[A, B]=A B-B A$. Denote por $E_{i j}$ a matriz de ordem $p+2$ cuja única entrada não-nulo é 1 e ocorre na posição de $i$ ésima linha e $j$-ésima coluna. Daí podemos identificar os geradores de $\mathfrak{g}$ com $E_{i j}$ para $i, j \in\{1,2, \ldots, p+2\}$ como a seguir:

$$
X_{i}=E_{1, i+1}, \quad Y_{j}=E_{j+1, p+2}, \quad Z=E_{1, p+2} .
$$

Desta forma, qualquer elemento de $G$ pode ser representado como uma combinação linear de $X_{i}, X_{j}$ e $Z$ tendo 1 na diagonal principal.

6. (Álgebra especial ortogonal $\mathfrak{s o}(3)$ ) As rotações do $\mathbb{R}^{3}$ em torno dos eixos coordenados são dadas pelas matrizes

$R_{1}(t)=\left(\begin{array}{ccc}1 & 0 & 0 \\ 0 & \cos t & -\sin t \\ 0 & \sin t & \cos t\end{array}\right), R_{2}(t)=\left(\begin{array}{ccc}\cos t & 0 & -\sin t \\ 0 & 1 & 0 \\ \sin t & 0 & \cos t\end{array}\right), R_{3}(t)=\left(\begin{array}{ccc}\cos t & -\sin t & 0 \\ \sin t & \cos t & 0 \\ 0 & 0 & 1\end{array}\right)$,

que certamente podem ser interpretadas como curvas no grupo $\mathrm{SO}(3)$ passando pela identidade. Tomanda a derivada delas na identidade obtem-se a álgebra de Lie $\mathfrak{s o}(3)$ de $\mathrm{SO}(3)$ cuja base é formada pelos seguintes campos vetoriais:

$$
Y^{1}=\left(\begin{array}{ccc}
0 & 0 & 0 \\
0 & 0 & -1 \\
0 & 1 & 0
\end{array}\right), Y^{2}=\left(\begin{array}{ccc}
0 & 0 & -1 \\
0 & 0 & 0 \\
1 & 0 & 0
\end{array}\right), Y^{3}=\left(\begin{array}{ccc}
0 & -1 & 0 \\
1 & 0 & 0 \\
0 & 0 & 0
\end{array}\right)
$$

Em outras palavras, $\mathfrak{s o}(3)$ é o conjunto de matrizes anti-simétricas em $\mathrm{M}_{3}(\mathbb{R})$ :

$$
\mathfrak{s o}(3)=\left\{A \in \mathfrak{s o}(3): A+A^{T}=0\right\} .
$$

Por outro lado, como $\left[Y^{1}, Y^{2}\right]=Y^{3}$, pode ser escrito também: $\mathfrak{s o}(3)=\operatorname{Lie}\left\{Y^{1}, Y^{2}\right\}$.

Definição 2.2.5. (Ideal) Um subespaço $\mathfrak{h} \subset \mathfrak{g}$ é um ideal se para todo $Y \in \mathfrak{h}, X \in \mathfrak{g}$ tem-se: $[X, Y] \in \mathfrak{h}$, isto é, $[\mathfrak{g}, \mathfrak{h}] \subset \mathfrak{h}$.

É claro que todo ideal é subálgebra. Porém, nem toda subálgebra é um ideal.

Definição 2.2.6. (Soma direta) Sejam $\mathfrak{g}_{1}, \ldots, \mathfrak{g}_{n}$ álgebras de Lie e $\mathfrak{g}=\mathfrak{g}_{1} \oplus \cdots \oplus \mathfrak{g}_{n}$ sua soma direta como espaços vetoriais. Isto é, $\mathfrak{g}=\mathfrak{g}_{1} \times \cdots \times \mathfrak{g}_{n}$ com a estrutura vetorial produto. Para $X=\left(X_{1}, \ldots, X_{n}\right)$ e $Y=\left(Y_{1}, \ldots, Y_{n}\right)$, a expressão

$$
[X, Y]=\left(\left[X_{1}, Y_{1}\right], \ldots,\left[X_{n}, Y_{n}\right]\right)
$$

define em $\mathfrak{g}$ uma estrutura de álgebra de Lie em que a i-ésima componente é um ideal isomorfo $a \mathfrak{g}_{i}$. 


\subsection{Derivações e Produtos Semidiretos}

Definição 2.3.1. (Derivação de álgebras de Lie) Uma aplicação linear $D: \mathfrak{g} \rightarrow \mathfrak{g} e ́$ denominada derivação, se

$$
D[X, Y]=[D X, Y]+[X, D Y]
$$

para todo $X, Y \in \mathfrak{g}$.

Denote por $\mathfrak{D} \mathfrak{e r}(\mathfrak{g})$ o espaço de todas as derivações de $\mathfrak{g}$, que é uma subálgebra de $\mathfrak{g l}(\mathfrak{g})$ pois $\left[D_{1}, D_{2}\right]=D_{1} D_{2}-D_{2} D_{1}$ é uma derivação para todo $D_{1}, D_{2} \in \mathfrak{D} \mathfrak{e r}(\mathfrak{g})$. Por exemplo, o operador de diferenciação parcial de primeira ordem

$$
X=\sum_{i=1}^{n} a_{i} \frac{\partial}{\partial x_{i}},
$$

onde $a_{i} \in C^{\infty}\left(\mathbb{R}^{n}\right)$, é uma derivação em $C^{\infty}\left(\mathbb{R}^{n}\right)$. Reciprocamente, toda derivação pertencente a $C^{\infty}\left(\mathbb{R}^{n}\right)$ é desta forma.

Como outro exemplo, dada um elemento $X$ na álgebra de Lie $\mathfrak{g}$, considere a transformação linear

$$
\operatorname{ad}(X): \mathfrak{g} \longrightarrow \mathfrak{g}
$$

definida por $\operatorname{ad}(X)(Y)=[X, Y]$, que é denominada representação adjunta de $\mathfrak{g}$.

Observação 2.3.2. Se $\mathfrak{h} \subset \mathfrak{g}$ é uma subálgebra e $X \in \mathfrak{h}$, a notação $\operatorname{ad}(X)$ pode significar tanto uma transformação linear de $\mathfrak{g}$ quanto de $\mathfrak{h}$. Se for preciso distinguir esses dois casos, o usual é indicar a álgebra com um subíndice. Por exemplo, $\operatorname{ad}_{\mathfrak{h}}(X)$ é uma transformação linear de $\mathfrak{h}$.

A propriedade de Jacobi para colchetes em álgebras de Lie garante que as aplicações $\operatorname{ad}(X), X \in \mathfrak{g}$, são as derivações de $\mathfrak{g}$. Elas são denominadas de derivações internas de $\mathfrak{g}$ e o conjunto destas derivações coincide com a imagem da representação adjunta. O espaço

$$
\operatorname{ad}(\mathfrak{g})=\{\operatorname{ad}(X) \in \mathfrak{g l}(\mathfrak{g}): X \in \mathfrak{g}\}
$$

das derivações internas é, portanto, uma subálgebra de $\mathfrak{D} \mathfrak{e r}(\mathfrak{g})$. Na verdade, $\operatorname{ad}(\mathfrak{g})$ é um ideal de $\mathfrak{D} \mathfrak{e r}(\mathfrak{g})$. De fato, se $D$ é uma derivação e $X \in \mathfrak{g}$ então a definição de derivação é equivalente a

$$
[D, \operatorname{ad}(X)]=\operatorname{ad}(D X) .
$$

Exemplo 2.3.3. Seja $\mathfrak{g}=\operatorname{Span}\left\{Y^{1}, Y^{2}, Y^{3}\right\}$ a álgebra de Lie de Heisenberg. Vamos encontrar as álgebras $\mathfrak{D} \mathfrak{e r}(\mathfrak{g})$ e $\operatorname{ad}(\mathfrak{g})$ das derivações e derivações internas, respectivamente. 
Seja $D: \mathfrak{g} \rightarrow \mathfrak{g}$ uma transformação linear de $\mathfrak{g}$ cuja representação matricial é $[D]=\left(d_{i j}\right)_{1 \leq i, j \leq 3}$.

É evidente que $D\left[Y^{i}, Y^{i}\right]=0$ para todo $i=1,2,3$ e que $D$ satisfaz

$$
D\left[Y^{1}, Y^{2}\right]=D\left(Y^{3}\right)=\left[Y^{1}, D Y^{2}\right]+\left[D Y^{1}, Y^{2}\right]
$$

se, e somente, se

$$
d_{13} Y^{1}+d_{23} Y^{2}+d_{33} Y^{3}=\left(d_{11}+d_{22}\right) Y^{3}
$$

Daí, segue que $d_{13}=d_{23}=0$ e $d_{11}+d_{22}=d_{33}$.

Da mesma forma, $D\left[Y^{1}, Y^{3}\right]=0=\left[Y^{1}, D Y^{3}\right]+\left[D Y^{1}, Y^{3}\right]=d_{23} Y^{3}$ se, se somente, se $0=-d_{13} Z$ o que resulta $d_{13}=0$.

Devido a anti-simetria do colchetede Lie $[\cdot, \cdot]$ essas condições obtidas para entradas de matriz $D$ não vão mudar e, portanto, uma derivação de álgebra de Heisenberg $\mathfrak{g}$ terá a forma

$$
[D]=\left(\begin{array}{ccc}
d_{11} & d_{12} & 0 \\
d_{21} & d_{22} & 0 \\
d_{31} & d_{32} & d_{11}+d_{22}
\end{array}\right)
$$

A conclusão disso é que a dimensão de álgebra $\mathfrak{D} \mathfrak{e r}(\mathfrak{g})$ das derivações de $\mathfrak{g}$ é 6 justamente porque há 6 parâmetros diferentes na matriz acima. Daí, a álgebra

$$
\mathfrak{D e r}(\mathfrak{g})=\operatorname{Span}\left\{D_{1}, D_{2}, D_{3}, D_{4}, D_{5}, D_{6}\right\}
$$

onde

$$
\begin{aligned}
D_{1}=\left(\begin{array}{lll}
1 & 0 & 0 \\
0 & 0 & 0 \\
0 & 0 & 1
\end{array}\right) & D_{2}=\left(\begin{array}{lll}
0 & 1 & 0 \\
0 & 0 & 0 \\
0 & 0 & 0
\end{array}\right) & D_{3}=\left(\begin{array}{lll}
0 & 0 & 0 \\
1 & 0 & 0 \\
0 & 0 & 0
\end{array}\right) \\
D_{4}=\left(\begin{array}{lll}
0 & 0 & 0 \\
0 & 1 & 0 \\
0 & 0 & 1
\end{array}\right) & D_{5}=\left(\begin{array}{lll}
0 & 0 & 0 \\
0 & 0 & 0 \\
1 & 0 & 0
\end{array}\right) & D_{6}=\left(\begin{array}{lll}
0 & 0 & 0 \\
0 & 0 & 0 \\
0 & 1 & 0
\end{array}\right) .
\end{aligned}
$$

Quanto às derivações internas, são apenas 2:

$$
\operatorname{ad}\left(Y^{1}\right)=\left(\begin{array}{lll}
0 & 0 & 0 \\
0 & 0 & 0 \\
0 & 1 & 0
\end{array}\right) \quad \text { e } \quad \operatorname{ad}\left(Y^{2}\right)=\left(\begin{array}{ccc}
0 & 0 & 0 \\
0 & 0 & 0 \\
-1 & 0 & 0
\end{array}\right)
$$

e portanto, $\operatorname{ad}(\mathfrak{g})=\operatorname{Span}\left\{D_{5}, D_{6}\right\}$.

Como nem toda derivação é interna existe derivações no quociente $\mathfrak{D} \mathfrak{e r}(\mathfrak{g}) / \operatorname{ad}(\mathfrak{g})$ que são chamadas de derivações externas. Note-se que, em geral, a diferença de cardinalidade entre derivações internas e externas pode ser grande. 
O núcleo da representação adjunta de $\mathfrak{g}$ é denominada de centro de $\mathfrak{g}$ e é denotado por $\mathfrak{z}(\mathfrak{g})$ :

$$
\mathfrak{z}(\mathfrak{g})=\{X \in \mathfrak{g}: \operatorname{ad}(X)(Y)=[X, Y]=0 \text { para todo } Y \in \mathfrak{g}\} .
$$

Isto é, o centro de uma álgebra de Lie é o conjunto de seus elementos que comutam com todos os seus elementos. Evidentemente, $\mathfrak{z}(\mathfrak{g})$ é um ideal de $\mathfrak{g}$. De forma mais geral,

Definição 2.3.4. (Centralizador) Sejam $\mathfrak{g}$ uma álgebra de Lie e A um subconjunto de g. O centralizador de $A$ em $\mathfrak{g}$, denotado por $\mathfrak{z}_{\mathfrak{g}}(A)$ é definido por

$$
\mathfrak{z}_{\mathfrak{g}}(A)=\{Y \in \mathfrak{g}:[X, Y]=0 \text { para todo } X \in A\}
$$

Para qualquer $A \subset \mathfrak{g}, \mathfrak{z}_{\mathfrak{g}}(A)$ é uma subálgebra, pois se $X, Y \in \mathfrak{z}_{\mathfrak{g}}(A)$ e $Z \in A$, então

$$
[[X, Y], Z]=[[X, Z], Y]+[X,[Y, Z]]=0
$$

pela identidade de Jacobi. No entanto, $\mathfrak{z}_{\mathfrak{g}}(A)$ nem sempre é um ideal como ocorre com o centro.

Definição 2.3.5. (Normalizador) Sejam $\mathfrak{g}$ uma álgebra de Lie e A um subconjunto de g. O normalizador de $A$ em $\mathfrak{g}$, denotado por $\mathfrak{n}_{\mathfrak{g}}(A)$ é definido por

$$
\mathfrak{n}_{\mathfrak{g}}(A)=\{X \in \mathfrak{g}: \operatorname{ad}(X) A \subset A\}
$$

Se $A$ é um subespaço vetorial de $\mathfrak{g}$ então segue da identidade de Jacobi que $\mathfrak{n}_{\mathfrak{g}}(A)$ é uma subálgebra de $\mathfrak{g}$. Em particular, $A$ é uma subálgebra de $\mathfrak{g}$ se, e só se, $A$ é um subespaço $A \subset \mathfrak{n}_{\mathfrak{g}}(A)$. Além do mais $A$ é um ideal de $\mathfrak{g}$ se $A$ é um subespaço vetorial e $\mathfrak{n}_{\mathfrak{g}}(A)=\mathfrak{g}$, isto é, se $[X, A] \subset A$ para todo $X \in \mathfrak{g}$.

Levando em conta o principio de que os grupos de Lie devem ser estudados através das álgebras de Lie, os homomorfismos entre grupos de Lie serão descritos através dos homomorfismos entre suas álgebras de Lie.

Definição 2.3.6. (Homomorfismo de álgebras de Lie) Sejam $\mathfrak{g}$ e hálgebras de Lie. Uma transformação linear $\theta: \mathfrak{g} \rightarrow \mathfrak{h}$ é dito um homomorfismo de álgebras de Lie se $\theta[X, Y]=[\theta X, \theta Y]$ para todo $X, Y \in \mathfrak{g}$. Diz-se que $\theta$ é um isomorfismo se for um homomorfismo inversivel e é automorfismo se é um isomorfismo com $\mathfrak{g}=\mathfrak{h}$.

Em particular, a aplicação ad $: \mathfrak{g} \rightarrow \mathfrak{g l}(\mathfrak{g})$ dada por $X \mapsto \operatorname{ad}(X)$ define um homomorfismo de álgebras de Lie. Denotaremos por $\operatorname{Aut}(\mathfrak{g})$ o grupo dos automorfismos de $\mathfrak{g}$, que é um subgrupo fechado do grupo linear $\operatorname{Gl}(\mathfrak{g})$. Portanto, Aut(g) é um grupo de Lie. Sua álgebra de Lie é formada pelas derivações de $\mathfrak{g}$. Para ver que $\mathfrak{D e r}(\mathfrak{g})$ é a álgebra 
de Lie de $\operatorname{Aut}(\mathfrak{g})$ basta verificar que $D$ é uma derivação de $\mathfrak{g}$ se, e somente, se $e^{t D}$ é automorfismo de $\mathfrak{g}$ para todo $t \in \mathbb{R}$. Mas, se $X, Y \in \mathfrak{g}$ então a derivada da igualdade $e^{t D}[X, Y]=\left[e^{t D} X, e^{t D} Y\right]$ em $t=0$ mostra que $D$ é derivação se $e^{t D}$ é automorfismo. Reciprocamente, se $D$ é derivação então as curvas

$$
\alpha(t)=e^{t D}[X, Y] \quad \text { e } \quad \beta(t)=\left[e^{t D} X, e^{t D} Y\right]
$$

satisfazem a equação diferencial linear $\gamma^{\prime}=D \gamma, \gamma \in \mathfrak{g}$, e têm a mesma condição inicial $\alpha(0)=[X, Y]=\beta(0)$. Portanto, $\alpha=\beta$ o que mostra que $e^{t D}$ é automorfismo para todo $t \in \mathbb{R}$. Temos provado então a seguinte

Proposição 2.3.7. Seja $\mathfrak{g}$ uma álgebra de Lie real de dimensão finita e $D: \mathfrak{g} \rightarrow \mathfrak{g}$ uma transformação linear. Então, $D \in \mathfrak{D} \mathfrak{e r}(\mathfrak{g})$ se, e somente, se $e^{t D} \in \operatorname{Aut}(\mathfrak{g})$ para todo $t \in \mathbb{R}$.

A relação entre os homomorfismos de grupos e álgebras de Lie é fornecida pela diferencial no elemento neutro.

Proposição 2.3.8. Sejam $G$ e $H$ grupos de Lie com álgebras de Lie $\mathfrak{g}$ e $\mathfrak{h}$, respectivamente. Seja $: \phi: G \rightarrow H$ um homomorfismo diferenciável. Então, d $\phi_{e}: \mathfrak{g} \rightarrow \mathfrak{h} e ́$ homomorfismo de álgebras de Lie.

Demonstração: Veja prova da Proposição 3.16, [22].

A Proposição acima significa que homomorfismos de grupos de Lie induzem homomorfismo entre as álgebras de Lie correspondentes. O procedimento inverso, isto é, a construção homomorfismos de grupos que "estendem" homomorfismos de álgebras de Lie, nem sempre é possível, isto é, se $G$ e $H$ são grupos de Lie com álgebras de Lie $\mathfrak{g}$ e $\mathfrak{h}$, respectivamente, e $\theta: \mathfrak{g} \rightarrow \mathfrak{h}$ é um homomorfismo, nem sempre existe um homomorfismo $\phi: G \rightarrow H$ tal que $\theta=d \phi_{e}$. Porém, é importante notarmos que quando $G$ é simplesmente conexo, todo homomorfismo cujo domínio é a álgebra de Lie $\mathfrak{g}$ de $G$ se estende a um homomorfismo diferenciável com domínio $G$. Esse resultado é conhecido como princípio da monodromia e apresentamos abaixo o seu enunciado cuja demonstração detalhada pode ser encontrada em (Teorema 5.15, [22]).

Teorema 2.3.9. (Teorema de extensão de homomorfismos) Sejam $G$ e $H$ dois grupos de Lie com álgebras de Lie $\mathfrak{g}$ e $\mathfrak{h}$, respectivamente. Suponha que $G$ seja conexo $e$ simplesmente conexo. Então, para todo homomorfismo $\theta: \mathfrak{g} \rightarrow \mathfrak{h}$ existe um único homomorfismo $\phi: G \rightarrow H$ tal que $\theta=d \phi_{e}$.

De acôrdo com o principio da monodromia os bons resultados de extensão de homomorfismos de álgebras de Lie a grupos de Lie são obtidos com a hipótese de que 
o domínio é um grupo simplesmente conexo. Esse resultado tem como consequência a unicidade dos grupos de Lie simplesmente conexos com uma álgebra de Lie dada. Pode ser definido um produto no recobrimento universal (recobrimento simplesmente conexo) de um grupo de Lie, tornando esse recobrimento um grupo de Lie, denotado por $\widetilde{G}$. Porém, não pretende-se detalhar aqui a construção da estrutura de grupo de Lie no recobrimento universal que resulta na demonstração de que as álgebras de Lie (ou melhor suas classes de equivalência) estão em bijeção com os grupos de Lie simplesmente conexos.

Representando uma álgebra de Lie em outra por derivações, pode-se construir uma álgebra de Lie no produto das duas álgebras. Esse produto, chamado de produto semidireto, é bastante semelhante ao produto semidireto de grupos e generaliza o produto direto de álgebras visto anteriormente.

Definição 2.3.10. (Produto semidireto de álgebras de Lie) Sejam $\mathfrak{g}$ e $\mathfrak{h}$ álgebras de Lie e $\rho: \mathfrak{g} \rightarrow \mathfrak{D e r}(\mathfrak{h})$ um homomorfismo de álgebras de Lie. O produto semi-direto $\mathfrak{g} \otimes \mathfrak{h}$ é a álgebra de Lie em $\mathfrak{g} \times \mathfrak{h}$ dada pelo colchete

$$
\left[\left(X_{1}, Y_{1}\right),\left(X_{2}, Y_{2}\right)\right]=\left(\left[X_{1}, X_{2}\right], \rho\left(X_{1}\right) Y_{2}-\rho\left(X_{2}\right) Y_{1}+\left[Y_{1}, Y_{2}\right]\right)
$$

Dado um produto semi-direto $G \otimes H$ sejam $\mathfrak{g}$ e $\mathfrak{h}$ as álgebras de Lie de $G$ e $H$, respectivamente. O homomorfismo $\tau: G \rightarrow \operatorname{Aut}(H)$ é diferenciável e sua diferencial $\rho=d \tau_{e}$ no elemento neutro é um homomorfismo $\rho: \mathfrak{g} \rightarrow \mathfrak{a} \mathfrak{u}(H)$ das álgebras de Lie correspondentes. Porém, aut $(H)$ é uma subálgebra de $\mathfrak{D} \mathfrak{e r}(\mathfrak{h})$.

Proposição 2.3.11. A álgebra de Lie de $G \otimes H$ é $\mathfrak{g} \otimes \mathfrak{h}$.

Uma álgebra de Lie $\mathfrak{l}$ é isomorfa a um produto semidireto $\mathfrak{g} \otimes \mathfrak{h}$ se, e somente, se $\mathfrak{l}=\mathfrak{g}_{1} \oplus \mathfrak{h}_{1}$ (soma direta de espaços vetoriais) tal que $\mathfrak{g}_{1}$ é uma subálgebra isomorfa a $\mathfrak{g}$ e $\mathfrak{h}_{1}$ é um ideal isomorfo a $\mathfrak{h}$.

A seguir será definida a álgebra de Lie de um grupo de Lie $G$ como uma subálgebra da álgebra de Lie $X(G)$ dos campos de vetores sobre $G$, formada por campos invariantes em $G$.

\subsection{Campos Invariantes}

Definição 2.4.1. Seja $G$ um grupo de Lie. Um campo de vetores $X$ em $G$ é dito invariante à direita se para todo $g \in G, d R_{g} \circ X=X \circ R_{g}$ (ou seja, $\left(d R_{g}\right)_{h} X(h)=X(h g)$ para todo $g, h \in G)$. O campo de vetores $X$ é invariante à esquerda se $d L_{g} \circ X=X \circ L_{g}$, para todo $g \in G$. 
Os campos invariantes à direita ou à esquerda são completamente determinados por seus valores no elemento neutro $e \in G$, pois para todo $g \in G$ a condição de invariança à direita, por exemplo, implica que $X(g)=\left(d R_{g}\right)_{e} X(e)$. Portanto, cada elemento do espaço tangente $T_{e} G$ determina um único campo invariante à direita e um único campo invariante à esquerda.

Dado $X \in T_{e} G$ a notação $X^{R}$ indica o campo invariante à direita tal que $X^{R}(e)=$ $X$. Já $X^{L}$ denota o campo invariante à esquerda correspondente. Explicitamente,

$$
X^{R}(g)=\left(d R_{g}\right)_{e}(X) \quad \text { e } \quad X^{L}(g)=\left(d L_{g}\right)_{e}(X) .
$$

Denote por $\operatorname{Inv}^{R}$ o conjunto dos campos invariantes à direita. Este conjunto é um subespaço vetorial real do espaço $X(G)$ de todos os campos de vetores em $G$, já que $\left(d R_{g}\right)$ é uma aplicação linear sobre os campos de vetores.

Analogamente, o conjunto $\operatorname{Inv}^{L}$ dos campos invariantes à esquerda também é um subespaço vetorial (em geral, diferente do subespaço dos campos invariantes à direita). As aplicações $X \in T_{e} G \mapsto X^{R} \in \operatorname{Inv}^{R}$ e $X \in T_{e} G \mapsto X^{L} \in \operatorname{Inv}^{L}$ são isomorfismos entre os espaços vetoriais correspondentes.

Exemplos 2.4.2. 1. Seja $G=\left(\mathbb{R}^{n},+\right)$. Fixando $v \in \mathbb{R}^{n}$, as translações à esquerda e à direita coincidem e são dadas por

$$
L_{v}(x)=R_{v}(x)=x+v .
$$

Portanto, $\left(d L_{v}\right)_{y}=\left(d R_{v}\right)_{y}=$ id para todo $y \in \mathbb{R}^{n}$. Isso significa que os campos invariantes são constantes, isto é, $X(v)=v$, com $v \in \mathbb{R}^{n}$ fixado. A equação diferencial correspondente é $d x / d t=v$ cujo fluxo é a translação $X_{t}(x)=x+t v$.

2. Seja $G=\operatorname{Gl}(n, \mathbb{R})$ o grupo linear geral, que é um conjunto aberto do espaço vetorial real $\mathrm{M}_{n}(\mathbb{R})$ das matrizes. Fixando $g \in G$, as translações à esquerda e à direita $L_{g}(h)=g h$ e $R_{g}(h)=h g$ são as restrições a $\operatorname{Gl}(n, \mathbb{R})$ de transformações lineares de $\mathrm{M}_{n}(\mathbb{R})=\mathbb{R}^{n^{2}}$. O fibrado tangente a $G$ se identifica com $G \times \mathrm{M}_{n}(\mathbb{R})$. Daí que um campo invariante de vetores $X$ em $G$ é nada mais nada menos que uma aplicação $X: G \rightarrow \mathrm{M}_{n}(\mathbb{R})$. Além do mais, por essa identificação, as transformações lineares $L_{g}$ e $R_{g}$ satisfazem $d\left(L_{g}\right)_{h}=L_{g}$ e $d\left(R_{g}\right)_{h}=R_{g}$ para quaisquer $g, h \in G$. A partir dessa observação é possível descrever os campos invariantes em $\operatorname{Gl}(n, \mathbb{R})$. Suponha que $X: G \rightarrow \mathrm{M}_{n}(\mathbb{R})$ é invariante à direita. Então, para todo $g \in G$,

$$
X(g)=d\left(R_{g}\right)_{e} X(e)=R_{g} X(e)=X(e) g .
$$

Portanto, os campos invariantes à direita são da forma $X(g)=A g \operatorname{com} A$ uma matriz em $T_{e} G$. A equação diferencial definida por $X$ é o sistema linear

$$
\frac{d g}{d t}=A g
$$


no espaço das matrizes. O fluxo (linear) de $X$ é dado por $X_{t}(g)=e^{t A} g$, onde $e^{A}=$ $\Sigma_{k \geq 0} \frac{1}{k !} A^{k}$ é a exponencial de matrizes. De forma análoga, os campos invariantes à esquerda são da forma $X(g)=g A$ que estão associados aos sistemas lineares $\frac{d g}{d t}=g A$. Os seus fluxos têm a forma: $X_{t}(g)=g e^{t A}$.

A álgebra de Lie de um grupo de Lie é definida em qualquer um dos espaços de campos invariantes $\operatorname{Inv}^{R}$ ou $\operatorname{Inv}^{L}$ munido com o colchete de Lie. Sejam $X$ e $Y$ campos invariantes à direita num grupo de Lie $G$. Então, o colchete de Lie $[X, Y]$ é invariante à direita. A mesma afirrmação vale para campos invariantes à esquerda. Ou seja, os espaços $\operatorname{Inv}^{R}$ e Inv ${ }^{L}$ são subálgebras de Lie da álgebra de Lie $X(G)$ de todos os campos de vetores em $G$. Em particular, ambos os espaços vetoriais admitem estruturas de álgebra de Lie. A álgebra de Lie do grupo $G$ é qualquer uma das álgebras de Lie $\operatorname{Inv}^{R}$ ou $\operatorname{Inv}^{L}$. Na verdade, essas álgebras de Lie são, em essência, as mesmas, isto é, são isomorfas, não existindo, portanto, nenhuma ambiguidade na terminologia.

O espaço tangente $T_{e} G$ é isomorfo tanto a $\operatorname{Inv}^{R}$ quanto a $\operatorname{Inv}^{L}$. Através dos isomorfismos o colchete de Lie restrito aos subespaços de campos invariantes induz colchetes $[\cdot, \cdot]_{R}$ e $[\cdot, \cdot]_{L}$ em $T_{e} G$, dados por $[X, Y]_{R}=\left[X^{R}, Y^{R}\right](e)$ e $[X, Y]_{L}=\left[X^{L}, Y^{L}\right](e)$, $X, Y \in T_{e} G$.

Lema 2.4.3. (Invariância de colchete de Lie) Sejam $X$ e $Y$ campos invariantes à esquerda (resp. à direita) num grupo de Lie G. Então, o colchete de Lie $[X, Y]$ é invariante à esquerda (resp. à direita).

Definição 2.4.4. A álgebra de Lie de $G$, denotada por $\mathfrak{g}$ ou $L(G)$, é qualquer uma das álgebras de Lie isomorfas $\operatorname{Inv}^{R}, \operatorname{Inv}^{L},\left(T_{e} G,[\cdot, \cdot]_{R}\right)$ ou ainda $\left(T_{e} G,[\cdot, \cdot]_{L}\right)$.

Qualquer uma dessas álgebras de Lie pode ser escolhida para representar $\mathfrak{g}$. A diferença principal está, é claro, na escolha entre invariança à direita e à esquerda.

Proposição 2.4.5. Dado um grupo de Lie $G$ com álgebra de Lie $\mathfrak{g}$. A aplicação $\theta: \mathfrak{g} \rightarrow T_{e} G$ definida por

$$
\theta(X)=X(e)
$$

é um isomorfismo linear. Consequentemente, $\operatorname{dim} \mathfrak{g}=\operatorname{dim} T_{e} G=\operatorname{dim} G$.

Demonstração: Sejam $X, Y \in \mathfrak{g}$ tais que $\theta(X)=\theta(Y)$. Dado um $g \in G$ arbitrário, temos:

$$
X(g)=\left(d L_{g}\right)_{e} X(e)=\left(d L_{g}\right)_{e} Y(e)=Y(g),
$$

o que implica que $X=Y$, ou seja, $\theta$ é injetora. Para ver sobrejetividade, seja $v \in T_{e} G$ e considere o campo vetorial $X$ em $G$ dado por $X(g)=\left(d L_{g}\right)_{e} v$, para todo $g \in G$. Segue então que $X$ é invariante à esquerda. 
A identificação de álgebra de Lie $\mathfrak{g}$ com o espaço tangente $T_{e} G$ ao grupo correspondente $G$ na identidade, nós dá informação local sobre $G$. De fato, os grupos de Lie localmente isomorfos possuem a mesma álgebra de Lie.

Lema 2.4.6. Sejam $X \in T_{e} G \simeq \mathfrak{g} e \iota(g)=g^{-1}$ a inversa em $G$. Então, d $\iota_{e} X^{R}=$ $(-X)^{L}$ (em detalhes: $d \iota_{g^{-1}} X^{R}\left(g^{-1}\right)=-\left(X^{L}\right)(g)$ e d $\iota_{e}\left(X^{L}\right)=(-X)^{R}$.

Demonstração: Usando a regra da cadeia e a igualdade $L_{g} \circ \iota=\iota \circ R_{g^{-1}}$, segue que $\left(d L_{g}\right)_{e}(Y)=d\left(\iota \circ R_{g^{-1}}\right)_{e} X^{R}$. Este segundo membro é igual a $d \iota_{e} X^{R}=Y$, pela a regra da cadeia e pelo fato que $X^{R}$ é invariante à direita. Portanto, $\left(d L_{g}\right)_{e}(Y)=Y$ e daí que $Y$ é invariante à esquerda. Agora, $Y(e)=d \iota_{e} X^{R}(e)$. $\operatorname{Mas} X^{R}(e)=X$ e $d \iota_{e}=-$ id (Proposição 2.1.3), daí que $Y(e)=-X$, mostrando que $Y=(-X)^{L}$. A outra igualdade é provada da mesma maneira, ou então aplicando $d \iota$ na igualdade já demonstrada.

\subsection{Aplicação Exponencial}

A aplicação exponencial exp $: \mathfrak{g} \rightarrow G$ é usada para transportar ao grupo de Lie $G$ propriedades de sua álgebra de Lie $\mathfrak{g}$. De fato, alguns problemas aparentamente difícies de resolver no grupo podem ser tratados usando a informação no nível de álgebra. A idéia é que os elementos de $\mathfrak{g}$ são campos invariantes em $G$ (e, portanto, EDOs em $G$ ) que possuem fluxos formados por difeomorfismos locais de $G$. Os elementos que formam esses fluxos se identificam a elementos de $G$ permitindo construir a partir de $X \in \mathfrak{g}$ um subgrupo a 1-parâmetro de $G$. A aplicação exponencial é construida a partir desses subgrupos.

Veja a Definição a seguir

Definição 2.5.1. Um homomorfismo diferenciável $\varphi: \mathbb{R} \rightarrow G$ é dito um subgrupo a 1-parâmetro de $G$.

Segue da definição que a aplicação $\varphi \rightarrow d \varphi_{0}(1)$ é uma correspondência biunívoca entre $G$ e seu espaço tangente.

Exemplo 2.5.2. Para todo $r \in \mathbb{R}$ e $\theta$ a aplicação

$$
\varphi(t)=\left(\begin{array}{cc}
\cos (t r) & e^{i \theta} \operatorname{sen}(t r) \\
-e^{i \theta} \operatorname{sen}(t r) & \cos (t r)
\end{array}\right)
$$

é um subgrupo a 1-parâmetro de $\mathrm{SU}(2)$, o grupo de Lie de matrizes $A$ com entradas complexas tais que $A A^{*}=I d$ onde $A^{*}$ denota o conjugado complexo de $A$. De fato, como $\varphi(s+t)=\varphi(s) \varphi(t)$ e $d \varphi_{0}(1)=A \in \mathfrak{s u}(2)$ podemos ver que $\varphi$ é mesmo um subgrupo a 1-parâmetro de $\mathrm{SU}(2)$. 
Proposição 2.5.3. Sejam $X \in \operatorname{Inv}^{R}$ e $g, h \in G$ tal que $h \in \operatorname{dom}\left(X_{t}\right)$. Então

$$
X_{t}(h g)=X_{t}(h) g .
$$

Em particular, quando $h=e \in G$ tem-se: $X_{t}(g)=X_{t}(e) g$. Analogamente, se $X \in \mathrm{Inv}^{L}$ então vale $X_{t}(g h)=g X_{t}(h)$.

A Proposição diz que a invariança de $X$ acarreta a simetria do seu fluxo $X_{t}$ no sentido de que a solução que passa por $g \in G$ é obtida por translação da solução que passa pelo elemento neutro do grupo. Por outro lado, como as trajetórias são obtidas umas das outras por translação elas se prolongam sobre todo $\mathbb{R}$ e consequentemente os campos invariantes são completos. Veja Proposição 3.7 de [22].

Agora definimos a aplicação exponencial.

Definição 2.5.4. Seja $X \in T_{e} G$. Então, $\exp X=X_{t}^{R}(e)=X_{t}^{L}(e)$. Como é usual exp $X$ também se escreve $e^{X}$.

A aplicação $t \mapsto \exp (t X), X \in \mathfrak{g}$, é um homomorfismo e, portanto, $\{\exp (t X): t \in$ $\mathbb{R}\}$ é um subgrupo a 1-parâmetro de $G$ no sentido da Definição 2.5.1. Note também que para grupos abelianos, exp é um homomorfismo mas o mesmo não vale em geral. A relação entre o produto $\exp X \exp Y \operatorname{e} \exp (X+Y)$ é dada pela formula de CampbellBaker-Hausdorf

$$
\exp t X \exp t Y=\exp \left(t(X+Y)+\frac{t^{2}}{2}[X, Y]+\frac{t^{3}}{12}([[X, Y], Y]-[[X, Y], X])+O\left(t^{4}\right)\right)
$$

Proposição 2.5.5. Se $X \in \operatorname{Inv}^{L}$, isto é, se $X$ é um campo invariante à esquerda, $e$ $g \in G$ então

$$
X_{t}(g)=g \exp (t X)
$$

Da forma similar, se $X \in \operatorname{Inv}^{R}$ então vale $X_{t}(g)=\exp (t X) g$.

Proposição 2.5.6. $d(\exp )_{0}=\mathrm{id}$.

Demonstração: Dado $X \in \mathfrak{g},(\exp )_{0}(X)=\left(\frac{d}{d t}\right)_{t=0} \exp (0+t X)$. Mas essa derivada é exatamente $X$ pois a curva $\exp (t X)$ é a solução de $d g / d t=X^{R}(g)$. Portanto, $d(\exp )_{0}(X)=X$, concluindo a demonstração.

Corolário 2.5.7. Existem uma vizinhança $U$ de $0 \in \mathfrak{g}$ e uma vizinhança $V$ de e em $G$ tal que $\left.\exp \right|_{U}: U \rightarrow V$ é um difeomorfismo.

Corolário 2.5.8. Seja $G$ um grupo de Lie conexo e tome $g \in G$. Então, existem $X_{1}, \ldots, X_{k} \in \mathfrak{g}$ tal que

$$
g=\exp X_{1} \cdots \exp X_{k}
$$


Demonstração: Como $G$ é conexo, a vizinhança $V$ do Corolário anterior gera $G$, isto é,

$$
G=\bigcup_{n \geq 1} V^{n}
$$

Um elemento de $V^{n}$ é da forma $g_{1} \cdots g_{n} \operatorname{com} g_{i}=\exp X_{i} \in V$. Portanto, um elemento de $V^{n}$ é um produto de exponenciais, o mesmo ocorrendo com $g \in G$ arbitrário.

Proposição 2.5.9. Sejam $G$ e $H$ grupos de Lie com álgebras de Lie $\mathfrak{g}$ e $\mathfrak{h}$, respectivamente. Seja $\phi: G \rightarrow H$ um homomorfismo diferenciável e tome $X \in \mathfrak{g}$. Então,

$$
\phi(\exp (X))=\exp \left(d \phi_{e}(X)\right)
$$

Demonstração: Considere a curva $\alpha: \mathbb{R} \rightarrow H$ dada por $\alpha(t)=\phi(\exp (t X))$. Essa é uma curva diferenciável em $H$ cuja derivada em 0 é $d \phi_{e} X(e)$. Também é um subgrupo a 1-parâmetro de $H$ já que $\phi$ é um homomorfismo. Por outro lado, $t \rightarrow \exp t(d \phi(X))$ é o único subgrupo a 1-parâmetro de $H$ cuja derivada em 0 é $d \phi(X)(e)$. Portanto,

$$
\phi(\exp t X)=\exp t(d \phi(X))
$$

para todo $t$. Em particular, tem-se:

$$
\phi(\exp (X))=\exp \left(d \phi_{e}(X)\right)
$$

Exemplo 2.5.10. Considere as aplicações det $: \operatorname{Gl}(n, \mathbb{R}) \rightarrow \mathbb{R}, A \mapsto \operatorname{det}(A)$, e $\operatorname{tr}$ : $\mathrm{M}_{n}(\mathbb{R}) \rightarrow \mathbb{R}, A \rightarrow \operatorname{tr} A$ (i.e, traço da matriz $A$ ). Segue então que para todo $A \in \mathrm{M}_{n}(\mathbb{R})$ tem-se:

$$
e^{\operatorname{tr} A}=\operatorname{det}(\exp A)
$$

De fato, no caso particular em que $A$ é uma matriz triangular superior com entradas diagonais $\lambda_{1}, \ldots, \lambda_{n}$ a matriz $A^{k}$ é também uma matriz triangular superior com entradas diagonais $\lambda_{1}^{k}, \ldots, \lambda_{n}^{k}$. O mesmo vale para $\exp A$, ou seja, $\exp A$ é uma matriz triangular superior com entradas diagonais $e^{\lambda_{1}}, \ldots, e^{\lambda_{n}}$ e que determinante dessa matriz

$$
\operatorname{det}(\exp A)=e^{\lambda_{1}} e^{\lambda_{2}} \cdots e^{\lambda_{n}}=e^{\lambda_{1}+\lambda_{2}+\cdots+\lambda_{n}}
$$

é igual ao traço de matriz $A$ conforme visto em (2.6). Em geral, tem-se: $A=$ $P T P^{-1}$ com $T$ sendo uma matriz triangular superior. Daí, $\exp A=\exp \left(P T P^{-1}\right)=$ $P(\exp T) P^{-1}$ e, portanto, $\operatorname{det}(\exp A)=\operatorname{det}(\exp T)$ o que implica que $\operatorname{tr} A=\operatorname{tr} T$. 



\section{Capítulo}

\section{3}

\section{Sistemas Lineares em $\mathbb{R}^{n}$}

Definição 3.0.11. Um sistema de controle linear em $\mathbb{R}^{n}$ é dado por $\Sigma=\left(\mathbb{R}^{n}, \mathcal{D}\right)$, onde $\mathbb{R}^{n}$ é o espaço estado e $\mathcal{D}$ é a dinâmica determinada pela seguinte família de equações diferenciais ordinárias em $\mathbb{R}^{n}$

$$
\dot{x}(t)=A x(t)+B u(t)
$$

sendo $A \in M_{n}(\mathbb{R}), B \in M_{n \times m}(\mathbb{R})$ e $\mathcal{U}_{m}$ é a classe de controles $u:[0, \infty) \rightarrow \mathbb{R}^{m}$ admissiveis constantes por pedaços a valores em $\mathbb{R}^{m}$.

Em outras palavras, a dinâmica do sistema $\Sigma$ é dada por

$$
\mathcal{D}=\left\{A x+B u \mid u \in \mathcal{U}_{m}\right\} .
$$

\subsection{Exemplos}

Exemplo 3.1.1. Considere $\Sigma=\left(\mathbb{R}^{2}, \mathcal{D}\right)$ dado por

$$
\begin{aligned}
& \dot{x}(t)=x(t)+y(t)+u(t), \quad u \in U_{1} . \\
& \dot{y}(t)=-y(t)
\end{aligned}
$$

Podemos escrever esse sistema de equações diferenciais como:

$$
\Sigma:\left(\begin{array}{l}
\dot{x} \\
y
\end{array}\right)=\left(\begin{array}{cc}
1 & 1 \\
0 & -1
\end{array}\right)\left(\begin{array}{l}
x \\
y
\end{array}\right)+\left(\begin{array}{l}
1 \\
0
\end{array}\right) u, \quad u \in U_{1} .
$$

Então $\Sigma$ é um sistema linear em $\mathbb{R}^{2}$. 
Exemplo 3.1.2. Considere $\Sigma=\left(\mathbb{R}^{2}, \mathcal{D}\right)$ determinado por

$$
\begin{aligned}
& \dot{x}(t)=x(t)+y(t) \\
& \dot{y}(t)=-y(t)+u(t)
\end{aligned}, \quad u \in U_{1} .
$$

Podemos escrever o sistema da seguinte forma:

$$
\Sigma:\left(\begin{array}{l}
\dot{x} \\
y
\end{array}\right)=\left(\begin{array}{cc}
1 & 1 \\
0 & -1
\end{array}\right)\left(\begin{array}{l}
x \\
y
\end{array}\right)+\left(\begin{array}{l}
0 \\
1
\end{array}\right) u, \quad u \in U_{1} .
$$

$\Sigma$ é um sistema de controle linear em $\mathbb{R}^{2}$.

Exemplo 3.1.3. Em $\mathbb{R}^{3}$ considere $\Sigma=\left(\mathbb{R}^{3}, \mathcal{D}\right)$ determinado por

$$
\begin{aligned}
& \dot{x}(t)=x(t)+y(t) \\
& \dot{y}(t)=y(t)+u(t), \quad u \in U_{1} . \\
& \dot{z}(t)=z(t)+u(t)
\end{aligned}
$$

O sistema de equações pode ser escrito como:

$$
\Sigma:\left(\begin{array}{l}
\dot{x} \\
y \\
z
\end{array}\right)=\left(\begin{array}{lll}
1 & 1 & 0 \\
0 & 1 & 0 \\
0 & 0 & 1
\end{array}\right)\left(\begin{array}{l}
x \\
y \\
z
\end{array}\right)+\left(\begin{array}{l}
0 \\
1 \\
1
\end{array}\right) u, \quad u \in U_{1}
$$

Então, o sistema $\Sigma=\left(\mathbb{R}^{3}, \mathcal{D}\right)$ é um sistema linear em $\mathbb{R}^{3}$.

Exemplo 3.1.4. Em $\mathbb{R}^{2}$, considere as matrizes

$$
A=\left(\begin{array}{cc}
0 & 1 \\
-1 & 0
\end{array}\right), \quad B=\left(\begin{array}{c}
1 \\
-1
\end{array}\right)
$$

Assim o sistema $\Sigma=\left(\mathbb{R}^{2}, \mathcal{D}\right)$ dado por

$$
\Sigma:\left(\begin{array}{l}
\dot{x} \\
y
\end{array}\right)=\left(\begin{array}{cc}
0 & 1 \\
-1 & 0
\end{array}\right)\left(\begin{array}{l}
x \\
y
\end{array}\right)+\left(\begin{array}{c}
1 \\
-1
\end{array}\right) u, \quad u \in U_{1} .
$$

é um sistema linear em $\mathbb{R}^{2}$.

Exemplo 3.1.5. Considere $\Sigma=\left(\mathbb{R}^{2}, \mathcal{D}\right)$ determinado por

$$
\begin{aligned}
& \dot{x}(t)=y(t) \\
& \dot{y}(t)=x(t)+u(t), \quad u \in U_{1} .
\end{aligned}
$$

Esse sistema de equações diferenciais pode ser ecrito como:

$$
\Sigma:\left(\begin{array}{l}
\dot{x} \\
y
\end{array}\right)=\left(\begin{array}{cc}
0 & 1 \\
-1 & 0
\end{array}\right)\left(\begin{array}{l}
x \\
y
\end{array}\right)+\left(\begin{array}{l}
0 \\
1
\end{array}\right) u, \quad u \in U_{1} .
$$

$\Sigma$ é claramente um sistema linear em $\mathbb{R}^{2}$. 
Dado $u(\cdot) \in \mathcal{U}_{m}$, sabe-se (veja, por exemplo, Coddington-Levinson [8]) que a solução do sistema (3.1) gerada pelo controle $u$ a partir de condição inicial $x_{0} \in \mathbb{R}^{n}$ é dada pela fórmula de variação de parâmetro

$$
x(t)=x\left(t, x_{0}, u\right)=\Phi(t) x_{0}+\int_{0}^{t} \Phi(t-\tau) B u(\tau) d \tau .
$$

Aqui, $\Phi(t)=e^{t A}$ é a matriz fundamental de sistema homogêneo

$$
\dot{x}=A x .
$$

É importante notarmos que quando todas as potências de uma matriz $A$ comutam $\left(A^{p} \cdot A^{q}=A^{p+q}\right)$ tem-se

$$
e^{t A} \cdot e^{s A}=e^{(t+s) A}
$$

Porém, se $A$ e $B$ são duas matrizes que não comutam, temos em geral:

$$
e^{t A} \cdot e^{s B} \neq e^{t A+s B}
$$

Por outro lado, observe que a expressão

$$
x\left(t, x_{0}, u\right)=e^{t A} x_{0}+e^{t A} \int_{0}^{t} e^{-\tau A} B u(\tau) d \tau
$$

é formal. Frequentemente, é muito dificil calcular $e^{t A}$. Porém se conhecemos o espectro da matriz $A$ então podemos usar forma canônica de Jordan para seu cálculo.

A seguinte observação é além de ser simples não deixa de ser interessante. Pondo $u=0$, obtemos sistema livre do controle cuja solução é dada pelo primeiro termo do lado direito da equação (3.4). Por outro lado, se colocarmos $x_{0}=0$ a solução será dada pelo outro termo, isto é,

$$
x\left(t, x_{0}, 0\right)=\int_{0}^{t} e^{(t-\tau) A} B u(\tau) d \tau .
$$

Por conseguinte, observamos que a solução de um sistema de controle linear (3.1) é a soma de solução do sistema homogêneo (3.3) com a condição inicial $x_{0}$ e solução de sistema não-homogêneo (3.1) com a condição inicial zero.

\subsection{Condição do Posto de Kalman}

Definição 3.2.1. O conjunto de pontos acessiveis do sistema $\Sigma$ a partir de $x_{0}$ em até o tempo $t>0$ é definido como:

$$
\mathcal{A}_{\Sigma}\left(x_{0}, t\right)=\left\{x\left(t, x_{0}, u\right) \mid u \in \mathcal{U}_{m}\right\}
$$


Veremos a seguir na Proposição 3.2.7 que o conjunto acessível de um sistema de controle linear em $\mathbb{R}^{n}$ é uma soma-vetorial de posição de movimento livre de controle $(u=0)$ começando em $x_{0}$ e mais o conjunto acessível a partir da origem.

Proposição 3.2.2. Se $0 \leq t_{1} \leq t_{2}$, então $\mathcal{A}_{\Sigma}\left(0, t_{1}\right) \subset \mathcal{A}_{\Sigma}\left(0, t_{2}\right)$

Demonstração: Seja $y \in \mathcal{A}_{\Sigma}\left(0, t_{1}\right)$, então

$$
y=x\left(t_{1}, 0, u\right), \quad \text { por algum } u(\cdot) \in \mathcal{U}_{m}
$$

Vamos definir o controle $u_{1}$ por

$$
u_{1}(s)=\left\{\begin{array}{lll}
0, & \text { se } & 0<s \leq t_{2}-t_{1} \\
u, & \text { se } & t_{2}-t_{1}<s \leq t_{2}
\end{array}\right.
$$

Assim

$$
x\left(s, 0, u_{1}\right)=\left\{\begin{array}{lll}
0, & \text { se } & 0<s \leq t_{2}-t_{1} \\
x(s, 0, u) & \text { se } & t_{2}-t_{1}<s \leq t_{2}
\end{array}\right.
$$

a saber, permanecemos parado na origem durante $t_{2}-t_{1}$ unidades de tempo e depois continuamos com o controle $u$. Portanto temos que,

$$
y=x\left(t_{2}, 0, u_{1}\right) \in \mathcal{A}_{\Sigma}\left(0, t_{2}\right)
$$

Note que a aplicação

$$
u \longrightarrow x\left(t, x_{0}, u\right)
$$

que envia um controle $u(\cdot)$ ao ponto final de sua trajetória é uma aplicação afim. Portanto, o conjunto acessível $\mathcal{A}_{\Sigma}\left(x_{0}, t\right)$ de um sistema linear em $\mathbb{R}^{n}$ em algum tempo fixo $t>0$ é um subespaço afim de $\mathbb{R}^{n}$.

Em particular, quando $x_{0}=0 \in \mathbb{R}^{n}$ tem-se a seguinte

Proposição 3.2.3. O conjunto acessível $\mathcal{A}_{\Sigma}(0, t)$ é um subespaço vetorial fechado de $\mathbb{R}^{n}$.

Demonstração: De fato, se $u_{1}(\cdot)$ e $u_{2}(\cdot)$ são controles admissíveis cujas trajetórias a partir de origem são $x_{1}(t)$ e $x_{2}(t)$, respectivamente, então $c_{1} u_{1}(t)+c_{2} u_{2}(t)$ também é um controle admissível para todo $c_{1}, c_{2} \in \mathbb{R}$.

Pela linearidade, a trajetótia correspondente é nada mais nada menos que $c_{1} x_{1}(t)+$ $c_{2} x_{2}(t)$. A conclusão é que se $x_{1}(t)$ e $x_{2}(t)$ são acessíveis a partir de origem em tempo $t>0$ então qualquer combinação linear $c_{1} x_{1}(t)+c_{2} x_{2}(t)$ também é acessível em $t>0$. 
Definição 3.2.4. A órbita positiva $S_{\Sigma}\left(x_{0}\right)$ do sistema $\Sigma$ a partir de $x_{0}$ é definida como:

$$
S_{\Sigma}\left(x_{0}\right)=\bigcup_{t>0} \mathcal{A}_{\Sigma}\left(x_{0}, t\right)
$$

Segue que $S_{\Sigma}(0)$ é um subespaço vetorial de $\mathbb{R}^{n}$.

Um dos principais problemas da teoria de controle é, determinar as órbitas positivas do sistema. Porém, é muito dificil computar $S_{\Sigma}(0)$ integrando a família de equações diferenciais que são dados como elementos da dinâmica $\mathcal{D}$. Abaixo introduzimos a noção de controlabilidade e apresentamos posteriormente uma condição de natureza algébrica conhecida como condição de Kalman para decidir quando temos controlabilidade de nosso sistema.

Definição 3.2.5. Seja $\Sigma$ um sistema linear em $\mathbb{R}^{n}$ e $x_{0} \in \mathbb{R}^{n}$. O sistema $\Sigma$ é dito:

1. Controlável a partir de $x_{0}$ se $S_{\Sigma}\left(x_{0}\right)=\mathbb{R}^{n}$

2. Controlável em $\mathbb{R}^{n}$ se $\Sigma$ é controlável a partir de $x_{0}$, para todo $x_{0} \in \mathbb{R}^{n}$.

A definição de controlabilidade acima significa que para quaisquer pontos $y, z \in \mathbb{R}^{n}$ existe um controle admissível $u(\cdot)$ tal que a solução correspondente $x(\cdot, y, u)$ de sistema alcança partindo de $y$ o ponto $z$ em $t$ unidades de tempo, ou seja, $x(0, y, u)=y$ e $x(t, y, u)=z$.

Uma alternativa para calcular $S_{\Sigma}(0)$ será dada em seguida por uma condição simples de natureza algébrica, conhecida como condição de posto de Kalman.

Seja $V \subset \mathbb{R}^{n}$ um subespaço vetorial e $A \in M_{n}\left(\mathbb{R}^{n}\right)$ uma matriz. $V$ é dito $A$ invariante se $A(V) \subset V$.

Denotamos por $\langle A \mid B\rangle$ o menor subespaço $A$-invariante de $\mathbb{R}^{n}$ que contém a imagem $B\left(\mathbb{R}^{m}\right)$ da matriz $B$. Provaremos que $\mathcal{A}_{\Sigma}(0, t)=\langle A \mid B\rangle$ para todo $t>0$ e, portanto, $S_{\Sigma}(0)=\langle A \mid B\rangle=\mathcal{A}_{\Sigma}(0, t)$.

Assim, os pontos acessíveis de $\Sigma$ estão em $\langle A \mid B\rangle$ e além disso qualquer elemento de $\langle A \mid B\rangle$ pode ser alcançado a partir da origem em algum tempo arbitrário positivo. Observe que estamos considerando no presente contexto apenas a classe de controles admissíveis $\mathcal{U}_{m}$ em $\mathbb{R}^{m}$ sem restrição.

Teorema 3.2.6. Seja $\Sigma$ um sistema linear em $\mathbb{R}^{n}$. Então,

$$
\mathcal{A}_{\Sigma}(0, t)=\langle A \mid B\rangle, \forall t>0
$$

Demonstração: Observamos primeiro que $\langle A \mid B\rangle$ satisfaz as seguintes propriedades:

1. $\langle A \mid B\rangle$ é topologicamente fechado. 
2. $\langle A \mid B\rangle$ é $A^{n}$-invariante para cada $n \in \mathbb{N}$.

3. $\langle A \mid B\rangle$ é $e^{t A}$-invariante para cada $t>0$.

4. $x(t, 0, u) \in\langle A \mid B\rangle$ para todo $t>0$ e $u() \in \mathcal{U}_{m}$.

O item 1 é claro pois, $\langle A \mid B\rangle$ é um subespaço do $\mathbb{R}^{n}$. Para verificar o item 2 , lembramos que, por definição, $\langle A \mid B\rangle$ é $A$-invariante, ou seja, $A(\langle A \mid B\rangle) \subset\langle A \mid B\rangle$. Portanto, tem-se: $A(A(\langle A \mid B\rangle)) \subset\langle A \mid B\rangle$ e, assim por diante.

Quanto ao item 3, sabemos que a exponencial da matriz $t A$ é dada por

$$
e^{t A}=\sum_{k \geq 0} \frac{t^{k} A^{k}}{k !}
$$

e essa série aplicada a cada $x \in\langle A \mid B\rangle$ converge em $\langle A \mid B\rangle$ uma vez que $\langle A \mid B\rangle$ é fechado e $A^{k}$-invariante para todo $k \geq 0$.

Para provar o item 4 seja $x(t, 0, u) \in \mathcal{A}_{\Sigma}(0, t)$. Assim

$$
x(t, 0, u)=e^{t A} \int_{0}^{t} e^{-\tau A} B u(\tau) d \tau .
$$

Sabemos que $u(\tau) \in \mathbb{R}^{m}$ e, portanto, $B u(\tau) \in B\left(\mathbb{R}^{m}\right)$. Como $\langle A \mid B\rangle$ é o menor subespaço $A$-invariante de $\mathbb{R}^{n}$ que contém $B\left(\mathbb{R}^{m}\right)$ então $B u(\tau) \in\langle A \mid B\rangle$. Por outro lado, como $\langle A \mid B\rangle$ é $e^{\tau A}$-invariante, tem-se também $e^{-\tau A} B u(\tau) \in\langle A \mid B\rangle$.

Por último, $\int_{0}^{t} e^{-\tau A} B u(\tau) d \tau \in\langle A \mid B\rangle$, pois a integral é o limite das somas de Riemann e como $\langle A \mid B\rangle$ é fechado, então esse limite pertence a $\langle A \mid B\rangle$.

Aplicando novamente o fato de que $\langle A \mid B\rangle$ é $e^{t A \text {-invariante, temos que }}$

$$
x(t, 0, u)=e^{t A} \int_{0}^{t} e^{-\tau A} B u(\tau) d \tau \in\langle A \mid B\rangle .
$$

Concluímos finalmente que

$$
\mathcal{A}_{\Sigma}(0, t) \subset\langle A \mid B\rangle, \quad \forall t>0 .
$$

E de (3.7) deduzimos que

$$
S_{\Sigma}(0) \subset\langle A \mid B\rangle \quad \forall t>0 .
$$

Vamos considerar a interseção dos subespaços

$$
\mathcal{I}=\bigcap_{t>0} \mathcal{A}_{\Sigma}(0, t)
$$

Como $\mathbb{R}^{n}$ possui dimensão finita e a interseção de subespaços vetoriais é ainda um subespaço vetorial, existe $t_{0}>0$ tal que

$$
0<t<t_{0} \Longrightarrow \mathcal{A}_{\Sigma}(0, t)=\mathcal{I} .
$$


Seja $u=u(t) \in \mathbb{R}^{m}$. Então, $x(t, 0, u) \in \mathcal{I}$ para todo $t \in\left(0, t_{0}\right)$. Logo,

$$
\left(\frac{d}{d t}\right)_{t} x(t, 0, u) \in \mathcal{I}, \quad \forall t \in\left(0, t_{0}\right)
$$

pois se o vetor tangente a curva $x(t)=x(t, 0, u)$ no ponto de partida não estivesse em $\mathcal{I}$, tal curva não estaria contida no subespaço $\mathcal{I}$.

Vamos calcular (3.12) para $t=0$,

$$
\begin{aligned}
\left(\frac{d}{d t}\right)_{t=0} x(t, 0, u) & =\left(\frac{d}{d t}\right)_{t=0} e^{t A} \int_{0}^{t} e^{-\tau A} B u(\tau) d \tau \\
& =\left(A e^{t A} \int_{0}^{t} e^{-\tau A} B u(\tau) d \tau+e^{t A} e^{-t A} B u(t)\right)_{t=0} \\
& =B u(0) \in \mathcal{I}
\end{aligned}
$$

Consideremos para cada $t \in\left(0, t_{0}\right)$ o seguinte controle

$$
u_{t}:[0, \infty) \rightarrow \mathbb{R}^{m}
$$

definido por

$$
u_{t}(s)= \begin{cases}u, & \text { se } \quad 0<s \leq t \\ 0, & \text { se } \quad t<s\end{cases}
$$

Para $0<s \leq t$ temos,

$$
x\left(s, 0, u_{t}\right)=e^{s A} \int_{0}^{s} e^{-\tau A} B u_{t}(\tau) d \tau=e^{s A} \int_{0}^{s} e^{-\tau A} B u(\tau) d \tau=x(s, 0, u) .
$$

Para $t<s$

$$
x\left(s, 0, u_{t}\right)=x(s, x(t, 0, u), 0)=e^{s A} x(t, 0, u)+e^{s A} \int_{0}^{s} e^{-\tau A} B \cdot 0 d \tau=e^{s A} x(t, 0, u) .
$$

Obtemos assim,

$$
x\left(s, 0, u_{t}\right)= \begin{cases}x(s, 0, u), & \text { se } \quad 0<s \leq t \\ e^{s A} x(t, 0, u) & \text { se } t<s\end{cases}
$$

Tomando $t$ e $s$ próximos a origem, vemos que a aplicação $\alpha$ definida por

$$
\alpha(t, s)=x\left(s, 0, u_{t}\right)
$$

possui sua imagem contida em $\mathcal{I}$. De fato,

$$
\alpha(t, s)=\left\{\begin{array}{l}
x(s, 0, u) \in \mathcal{I} \\
e^{s A} x(t, 0, u) \in \mathcal{I} \quad \text { para t próximo de } 0
\end{array}\right.
$$


Derivando parcialmente, temos para cada $k \geq 0$

$$
\begin{aligned}
\left(\frac{\partial^{k}}{\partial s^{k}}\right)_{s=0}\left(\frac{\partial \alpha}{\partial t}\right)_{t=0} & =\left(\frac{\partial^{k}}{\partial s^{k}}\right)_{s=0}\left(\left(\frac{\partial}{\partial t}\right)_{t=0} x\left(s, 0, u_{t}\right)\right) \\
& =\left(\frac{\partial^{k}}{\partial s^{k}}\right)_{s=0}\left(\left(\frac{\partial}{\partial t}\right)_{t=0} e^{s A} x(t, 0, u)\right) \\
& =\left(\frac{\partial^{k}}{\partial s^{k}}\right)_{s=0} e^{s A} B u(0) \\
& =\left(A^{k} e^{s A} B u(0)\right)_{s=0} \\
& =A^{k} B u(0) .
\end{aligned}
$$

Por $(3.15)\left(\frac{\partial^{k}}{\partial s^{k}}\right)_{s=0}\left(\frac{\partial \alpha}{\partial t}\right)_{t=0}=A^{k} B u(0) \in \mathcal{I}$.

Em particular, temos

$$
\langle A \mid B\rangle \in \mathcal{I}
$$

Assim,

$$
\langle A \mid B\rangle \in \mathcal{I}=\bigcap_{t>0} \mathcal{A}_{\Sigma}(0, t) \subset \bigcup_{t>0} \mathcal{A}_{\Sigma}(0, t)=S_{\Sigma}(0) .
$$

Portanto,

$$
\langle A \mid B\rangle \subset S_{\Sigma}(0)
$$

De (3.11) e (3.16) concluímos que

$$
\langle A \mid B\rangle=S_{\Sigma}(0)
$$

Por outro lado, $\langle A \mid B\rangle \subset \mathcal{I}=\bigcap_{t>0} \mathcal{A}_{\Sigma}(0, t)$ implica que para todo $t \in\left(0, t_{0}\right)$,

$$
\langle A \mid B\rangle \subset \mathcal{A}_{\Sigma}(0, t)
$$

e por (3.10), para $t>0$,

$$
\langle A \mid B\rangle=\mathcal{A}_{\Sigma}(0, t)
$$

Proposição 3.2.7. Temos que $\mathcal{A}_{\Sigma}\left(x_{0}, t\right)=e^{t A} x_{0}+\mathcal{A}_{\Sigma}(0, t)$

Demonstração: $\operatorname{Sejam~} x, y \in \mathcal{A}_{\Sigma}\left(x_{0}, t\right)$ então, existem $u_{1}, u_{2} \in \mathcal{U}_{m}$ tais que

$$
x=x\left(t, x_{0}, u_{1}\right)=e^{t A} x_{0}+e^{t A} \int_{0}^{t} e^{-\tau A} B u_{1}(\tau) d \tau=e^{t A} x_{0}+x\left(t, 0, u_{1}\right),
$$


e

$$
y=x\left(t, x_{0}, u_{2}\right)=e^{t A} x_{0}+e^{t A} \int_{0}^{t} e^{-\tau A} B u_{2}(\tau) d \tau=e^{t A} x_{0}+x\left(t, 0, u_{2}\right) .
$$

Assim, qualquer elemento de $\mathcal{A}_{\Sigma}\left(x_{0}, t\right)$ pode ser escrito como $e^{t A} x_{0}$ mais um elemento proveniente de $\mathcal{A}_{\Sigma}(0, t)$, ou seja,

$$
\mathcal{A}_{\Sigma}\left(x_{0}, t\right)=e^{t A} x_{0}+\mathcal{A}_{\Sigma}(0, t)
$$

Observação 3.2.8. $S_{\Sigma}\left(x_{0}\right)=\bigcup_{t>0} \mathcal{A}_{\Sigma}\left(x_{0}, t\right)$ e dado que $\mathcal{A}_{\Sigma}\left(x_{0}, t\right)=e^{t A} x_{0}+\mathcal{A}_{\Sigma}(0, t)$, então temos para cada $x_{0} \in \mathbb{R}^{n}$ que

$\Sigma$ é controlável a partir de $x_{0} \in \mathbb{R}^{n} \Longleftrightarrow \Sigma$ é controlável a partir de origem.

Temos então o seguinte resultado:

Teorema 3.2.9. Seja $\Sigma$ um sistema linear em $\mathbb{R}^{n}$. Para cada $x_{0} \in \mathbb{R}^{n}$,

$\Sigma$ é controlável desde $x_{0} \in \mathbb{R}^{n} \Longleftrightarrow \Sigma$ é controlável desde a origem $\Longleftrightarrow\langle A \mid B\rangle=\mathbb{R}^{n}$

\subsection{Matriz de Controlabilidade}

Vamos especificar um pouco mais o que é o subespaço $\langle A \mid B\rangle$. Pois estamos interessados em descrever uma generealização da classe de sistemas lineares em $\mathbb{R}^{n}$ a grupos de Lie arbitrários. Vamos introduzir então um conceito geral da teoria de sistemas de controle em variedades, restrito em nosso caso ao $\mathbb{R}^{n}$, para obtermos a condição do posto de Kalman que garante controlabilidade de sistemas lineares com controles irrestritos. Tal resultado depende de posto de uma matriz $n$ por $n m$, chamada matriz de controlabilidade, que é definida como:

$$
M=\left(\begin{array}{lllll}
B & A B & A^{2} B & \cdots & A^{n-1} B
\end{array}\right) .
$$

Definição 3.3.1. Dado $x \in \mathbb{R}^{n}$, a álgebra de Lie $L(\Sigma)$ de um sistema linear $\Sigma e ́$

$$
L(\Sigma)(x)=\operatorname{Lie}\left\{A x+B u: u \in \mathcal{U}_{m}\right\}
$$

Sejam $b_{1}, \ldots, b_{m} \in \mathbb{R}^{n}$ as colunas da matriz $B$. Afirmamos que $L(\Sigma)(x)$ contém os vetores da forma:

1. $A x \pm b_{j}$

2. $\pm A b_{j}$

3. $A\left(b_{j} \pm b_{i}\right)$ 
4. $A^{k+1} b_{j} \operatorname{com} i, j=1, \ldots, m$ e $k \in \mathbb{N}$

Para verificar $(1)$, tome para $j=1, \ldots, m$ o controle $u_{j}=(0, \ldots, 1, \ldots, 0)$ com a $j$-ésima linha igual a 1 . Assim, $B u_{j}=B_{j}$ e então $A x \pm B u_{j}=A x \pm b_{j} \in L(\Sigma)(x)$.

Para o item (2),

$$
\left[A x, b_{j}\right]=-A b_{j} \text { e }\left[b_{j}, A x\right]=A b_{j} \Longrightarrow \pm A b_{j} \in L(\Sigma)(x) .
$$

Por outro lado,

$$
\left[A x \pm b_{i}, A x \pm b_{j}\right]= \pm A\left(b_{i} \pm b_{j}\right) \in L(\Sigma)(x)
$$

enquanto que

$$
\left[A x+b_{i}, A b_{j}\right]=A^{2} b_{j} \in L(\Sigma)(x)
$$

e assim por diante.

O teorema de Cayley-Hamilton, afirma que não é preciso continuar o cálculo de forma indefinida em (4). Mais precisamente, o processo finaliza-se em $n-1$, pois o teorema diz que toda matriz é raiz de seu polinômio característico, ou seja,

$$
p(A)=A^{n}+a_{n-1} A^{n-1}+\cdots+a_{1} A+a_{0} I=0
$$

e portanto,

$$
A^{n}=-a_{n-1} A^{n-1}-\cdots-a_{1} A-a_{0} I .
$$

Consequentemente,

$$
L(\Sigma)(x)=\operatorname{Span}\left\{A x \pm b_{j}, A b_{j}, A^{2} b_{j}, \ldots, A^{n-1} b_{j} \mid j=1, \ldots, m\right\} .
$$

Em particular,

$$
\begin{aligned}
L(\Sigma)(0) & =\operatorname{Span}\left\{b_{j}, A b_{j}, A^{2} b_{j}, \ldots, A^{n-1} b_{j} \mid j=1, \ldots, m\right\} \\
& =\operatorname{Span}\left(B \quad A B \quad A^{2} B \quad \cdots \quad A^{n-1} B\right),
\end{aligned}
$$

que é o espaço gerado pelas colunas da matriz de controlabilidade $M$.

É claro que $B u_{j}=b_{j} \in\langle A \mid B\rangle$ e $A^{k} b_{j} \in\langle A \mid B\rangle$, para todo $j=1, \ldots, m$ e $k=$ $1, \ldots, n-1$. Isso implica que,

$$
L(\Sigma)(0) \subset\langle A \mid B\rangle
$$

Por outro lado,

$$
\langle A \mid B\rangle \subset L(\Sigma)(0)
$$

E assim,

$$
S_{\Sigma}(0)=\langle A \mid B\rangle=L(\Sigma)(0) .
$$

Enunciamos por fim o seguinte resultado clássico: 
Teorema 3.3.2. (Kalman, Ho e Narendra, [13]). Seja $\Sigma$ um sistema linear em $\mathbb{R}^{n}$. Então

$$
\Sigma \text { é controlável } \Longleftrightarrow \operatorname{posto}(M)=n \text {. }
$$

Com efeito,

$$
\begin{aligned}
\Sigma \text { é controlável } & \Longleftrightarrow L(\Sigma)(0)=\mathbb{R}^{n} \\
& \Longleftrightarrow \operatorname{Span}\left\{b_{j}, A b_{j}, A^{2} b_{j}, \ldots, A^{n-1} b_{j} \mid j=1, \ldots, m\right\}=\mathbb{R}^{n} \\
& \Longleftrightarrow \operatorname{posto}(M)=n .
\end{aligned}
$$

Observação 3.3.3. Se para um certo sistema de controle, um estado $x_{1}$ não for atingível a partir de um outro $x_{0}$, a causa pode ser de alguma forma uma das seguintes razões: (i) Os controles admissíveis não são fortes suficientes para superar outras "forças"do sistema, ou (ii) Todos os controles admissíveis agem em direções de tal modo que não importa o quanto forte o controle escolhido, $x(t)$ sempre fica numa superfície de dimensão mais baixa em $\mathbb{R}^{n+1}$. De fato, há exemplos onde este fenômeno ocorre. Para estudar tal comportamento geralmente basta descartar completamente a restrição sobre o magnitude de $u \in U$ pondo $U=\mathbb{R}^{m}$. Então o resultado acima de controlabilidade para sistemas lineares em $\mathbb{R}^{n}$ vale para controles irrestritos.

Exemplo 3.3.4. Considere o Exemplo 3.1.1. Observe que o posto da matriz

$$
\left(\begin{array}{ll}
B & A B
\end{array}\right)=\left(\begin{array}{ll}
1 & 1 \\
0 & 0
\end{array}\right)
$$

é igual a 1. Portanto o sistema $\Sigma=\left(\mathbb{R}^{2}, \mathcal{D}\right)$ não é controlável em $\mathbb{R}^{2}$. Vamos calcular $S_{\Sigma}(0)$. Seja $x_{0}=\left(x_{0}^{1}, x_{0}^{2}\right) \in \mathbb{R}^{2}$ então

$$
\mathcal{A}_{\Sigma}\left(x_{0}, t\right)=\left\{x\left(t, x_{0}, u\right): u \in U_{1}\right\},
$$

onde,

$$
x\left(t, x_{0}, u\right)=\left(\begin{array}{c}
e^{t} x_{0}^{1}+\frac{1}{2} x_{0}^{2}\left(e^{t}-e^{-t}\right)+u\left(e^{t}-t-1\right) \\
x_{0}^{2} e^{-t}
\end{array}\right) .
$$

Então

$$
\mathcal{A}_{\Sigma}\left(x_{0}, t\right)=\left\{\left(\begin{array}{c}
e^{t} x_{0}^{1}+\frac{1}{2} x_{0}^{2}\left(e^{t}-e^{-t}\right)+u\left(e^{t}-t-1\right) \\
x_{0}^{2} e^{-t}
\end{array}\right) \mid u \in U_{1}\right\}
$$

Em particular,

$$
\mathcal{A}_{\Sigma}(0, t)=\left\{\left(\begin{array}{c}
u\left(e^{t}-t-1\right) \\
0
\end{array}\right) \mid u \in U_{1}\right\}
$$

Portanto

$$
S_{\Sigma}(0)=\left\{(x, 0) \in \mathbb{R}^{2}\right\} \cong \mathbb{R}
$$


É interessante observar que uma troca simples de posição em coordenadas do parâmetro $u$ na edo do exemplo acima resulta um sistema linear cujas soluções agora preenchem o plano- $x y$ todo. Veja o exemplo a seguir onde desta vez a segunda coordenada de um estado está sujeto a presença de controle.

Exemplo 3.3.5. Considere o Exemplo 3.1.2. Observe que a matriz

$$
\left(\begin{array}{ll}
B & A B
\end{array}\right)=\left(\begin{array}{cc}
0 & 1 \\
1 & -1
\end{array}\right)
$$

que possui posto igual a 2 . Portanto o sistema $\Sigma=\left(\mathbb{R}^{2}, \mathcal{D}\right)$ é controlável em $\mathbb{R}^{2}$.

Exemplo 3.3.6. Considere o Exemplo 3.1.3. Observe que a matriz

$$
\left(\begin{array}{lll}
B & A B & A^{2} B
\end{array}\right)=\left(\begin{array}{lll}
0 & 1 & 2 \\
1 & 1 & 1 \\
1 & 1 & 1
\end{array}\right)
$$

tem posto igual a 2 . Logo o sistema $\Sigma=\left(\mathbb{R}^{3}, \mathcal{D}\right)$ não é controlável em $\mathbb{R}^{3}$.

Vamos calcular $S_{\Sigma}(0)$. Para isso temos

$$
e^{t A}=\left(\begin{array}{ccc}
e^{t} & t e^{t} & 0 \\
0 & e^{t} & 0 \\
0 & 0 & e^{t}
\end{array}\right) \text { e } \quad e^{-s A}=\left(\begin{array}{ccc}
e^{-s} & -s e^{-s} & 0 \\
0 & e^{-s} & 0 \\
0 & 0 & e^{-s}
\end{array}\right)
$$

Portanto,

$$
x\left(t, x_{0}, u\right)=\left(\begin{array}{c}
e^{t}\left(x_{0}^{1}+2 u e^{-t}+u e^{-t} t-2 u+t x_{0}^{2}+t u\right) \\
-e^{t}\left(-x_{0}^{2}+u e^{-t} t+u e^{-t}-u\right) \\
-e^{t}\left(-x_{0}^{3}+u e^{-t} t+u e^{-t}-u\right)
\end{array}\right),
$$

onde $x_{0}=\left(x_{0}^{1}, x_{0}^{2}, x_{0}^{3}\right) \in \mathbb{R}^{3}$. Em particular,

$$
x(t, 0, u)=\left(\begin{array}{c}
e^{t}\left(2 u e^{-t}+u e^{-t} t-2 u+t u\right) \\
-e^{t}\left(u e^{-t} t+u e^{-t}-u\right) \\
-e^{t}\left(u e^{-t} t+u e^{-t}-u\right)
\end{array}\right)
$$

Assim,

$$
S_{\Sigma}(0) \cong \mathbb{R}^{2}
$$

Exemplo 3.3.7. Considere o Exemplo 3.1.4. Observe que

$$
\left(\begin{array}{ll}
B & A B
\end{array}\right)=\left(\begin{array}{cc}
1 & -1 \\
-1 & -1
\end{array}\right)
$$

possui posto 2. Portanto o sistema $\Sigma=\left(\mathbb{R}^{2}, \mathcal{D}\right)$ é controlável em $\mathbb{R}^{2}$. 
Exemplo 3.3.8. Considere o Exemplo 3.1.5. Observe a matriz

$$
\left(\begin{array}{ll}
B & A B
\end{array}\right)=\left(\begin{array}{ll}
0 & 1 \\
1 & 0
\end{array}\right)
$$

tem posto 2. Portanto o sistema $\Sigma=\left(\mathbb{R}^{2}, \mathcal{D}\right)$ é controlável em $\mathbb{R}^{2}$.

Exemplo 3.3.9. Consideremos agora um outro problema da vida real, a saber, o problema de trem. O trem corre na trilha e objetivo é fazé-lo parar num destino predeterminado. Por exemplo, o destino pode ser pensado como a estação e por questão de simplicidade o pensamos na origem.

Para observar os movimentos de trem precisamos considerar duas coordenadas tipo $\alpha(t)=(x(t), y(t))$ onde $x(t)$ e $y(t)$ denotam respectivamente a distância e a velocidade de trem dependendo de tempo. Fisicamente falando, a razão para usarmos duas coordenadas é clara pois naturalmente precisamos saber onde estamos e com que velocidade estamos andando.

O controle $u(t)$ é uma função a valores reais. Sob esta informação, a dinâmica do nosso sistema é dada pela Lei de Newton: $F=m a$.

Como se sabe, a derivada de distância com respeito a tempo nós dá velocidade, isto é, $\dot{x}(t)=y(t)$ e a derivada de velocidade é aceleração, ou seja, o próprio controle. Portanto, tem-se: $\dot{y}(t)=u(t)$.

Temos então a família de equações diferenciais em $\mathbb{R}^{2}$ parametrizadas por $u \in U_{1}$ tal que:

$$
\begin{gathered}
\dot{x}(t)=y(t) \\
\dot{y}(t)=u(t) .
\end{gathered}
$$

Este sistema linear sobre $\mathbb{R}^{2}$ pode ser posto na forma matricial como:

$$
\left(\begin{array}{l}
\dot{x} \\
y
\end{array}\right)=\left(\begin{array}{ll}
0 & 1 \\
0 & 0
\end{array}\right)\left(\begin{array}{l}
x \\
y
\end{array}\right)+\left(\begin{array}{l}
0 \\
1
\end{array}\right) u .
$$

A solução para um controle constante $u \in U_{1}=[-1,1]$ com a condição $x_{0}=\left(x_{0}^{1}, x_{0}^{2}\right) \in$ $\mathbb{R}^{2}$ é dada por

$$
x\left(t, x_{0}, u\right)=\left(\frac{t^{2}}{2} u+x_{0}^{2} t+x_{0}^{1}, u t+x_{0}^{2}\right) .
$$

Portanto, as trajetórias se movem ao longo de parâbolas

$$
\mp 2 x=y^{2}+c
$$

onde $c$ é uma constante e a órbita do sistema a partir de $x_{0} \in \mathbb{R}^{2}$ é como

$$
S_{\Sigma}\left(x_{0}\right)=\left\{\left(\begin{array}{c}
\frac{t^{2}}{2} u+x_{0}^{2} t+x_{0}^{1} \\
u t+x_{0}^{2}
\end{array}\right): u \in \mathbb{R}\right\} .
$$


Em particular, temos a órbita a partir da origem:

$$
S_{\Sigma}(0)=\left\{\left(\begin{array}{c}
\frac{t^{2}}{2} u \\
u t
\end{array}\right): u \in \mathbb{R}\right\} .
$$

A matiz de controlabilidade

$$
M=\left(\begin{array}{ll}
B & A B
\end{array}\right)=\left(\begin{array}{ll}
0 & 1 \\
1 & 0
\end{array}\right)
$$

tem posto 2 e pelo teorema de Kalman, este sistema é controlável. De fato, temos

$$
\mathcal{A}_{\Sigma}(0, t)=\mathbb{R}^{2}
$$

e, portanto,

$$
S_{\Sigma}(0)=\mathbb{R}^{2}
$$

É interessante notar neste exemplo que cada ponto de $\mathbb{R}^{2}$ pode ser alcançado a partir da origem considerando apenas os controles extremos $u= \pm 1$. 
Capítulo

\section{Sistemas de Controle Lineares em Grupos de Lie}

Neste capítulo estudaremos a noção de um sistema de controle linear em um grupo de Lie $G$, introduzida por Ayala e Tirao em [3] como uma generalização natural de sistemas lineares em $\mathbb{R}^{n}$ através de normalizador de álgebra de Lie $\mathfrak{g}$ de $G$ na álgebra $X(G)$ de todos os campos diferenciáveis em $G$. Em particular, veremos a caracterização desse normalizador considerando uma identificação útil de campos com funções diferenciáveis em $G$ a valores em sua álgebra de Lie $\mathfrak{g}$. Terminaremos a exposição com um resultado de controlabilidade local desta classe.

\subsection{Motivação}

Começamos lembrando os sistemas lineares em $\mathbb{R}^{n}$ apresentada no capítulo anterior. Mostraremos a ideia de como generalizar a classe de sistemas em espaços euclideanos a grupos de Lie.

Seja $\Sigma$ um sistema linear em $\mathbb{R}^{n}$ dada pela dinâmica $\dot{x}=A x+B u$, onde $A, B$ são matrizes de dimensão apropriada, $u \in \mathcal{U}_{m}$ e $x \in \mathbb{R}^{n}$. Denote por $b_{1}, b_{2}, \ldots, b_{m}$ as colunas da matriz $B$. Daí o sistema em questão pode ser re-escrito como:

$$
\dot{x}=A x+\sum_{j=1}^{m} u_{j} b_{j} .
$$

Cada $b_{j}$ define um campo invariante $Y^{j}$ em $\mathbb{R}^{n}$ dado por

$$
Y^{j}(x)=b_{j}, \quad x \in \mathbb{R}^{n} .
$$


Note que um sistema de controle linear em $\mathbb{R}^{n}$ com o controle zero $u \equiv 0$ é nada mais nada menos que uma equação diferencial linear:

$$
\dot{x}=A x .
$$

Dado uma condição inicial $x_{0} \in \mathbb{R}$, a solução dessa EDO é dada pelo fluxo linear $x(t)=e^{t A} x_{0}$. De fato, toda matriz $A$ pode ser interpretada como um campo vetorial em $\mathbb{R}^{n}$ cujo fluxo

$$
A_{t}=e^{t A}, \quad t \in \mathbb{R},
$$

pertence ao grupo linear $\operatorname{Gl}(n, \mathbb{R})$ de todos os automorfismos de $\mathbb{R}^{n}$.

Por outro lado, uma computação simples mostra que

$$
\left[A x, b_{j}\right]=-A b_{j}
$$

para cada campo invariante $b_{j}$ em $\mathbb{R}^{n}$. Isto é, para cada campo invariante em $\mathbb{R}^{n}$ o colchete em (4.1) resulta por translação um campo invariante em $\mathbb{R}^{n}$. Em outras palavras, $A$ deixa invariante a álgebra de Lie $\mathbb{R}^{n}$ de $\mathbb{R}^{n}$. Achamos conveniente então apresentar a seguinte

Definição 4.1.1. Um campo $X$ em $\mathbb{R}^{n}$ é dito linear se $X(p)=A(p)+b$, onde $A$ : $\mathbb{R}^{n} \rightarrow \mathbb{R}^{n}$ é linear e b é um vetor fixo em $\mathbb{R}^{n}$.

Campos lineares em $\mathbb{R}^{n}$ podem ser caracterizados da seguinte forma: Um campo diferenciável $X$ em $\mathbb{R}^{n}$ é linear se, e somente, se para todo campo constante $Y$ em $\mathbb{R}^{n}$ o colchete $[X, Y]$ também é um campo constante. Todas essas observações indicam a presença de noção de normalizador. Temos portanto o seguinte

Lema 4.1.2. O normalizador da álgebra de Lie de $\mathbb{R}^{n}$ na álgebra de Lie $X\left(\mathbb{R}^{n}\right)$ de todos os campos diferenciáveis em $\mathbb{R}^{n}$ é o conjunto de todos os campos lineares em $\mathbb{R}^{n}$, ou seja,

$$
\mathfrak{n}_{X\left(\mathbb{R}^{n}\right)}\left(\mathbb{R}^{n}\right)=\left\{\text { campos lineares em } \mathbb{R}^{n}\right\} .
$$

Demonstração: (Cardetti, [6]) Sejam $X$ um campo linear em $\mathbb{R}^{n}$ e $Y$ um elemento da álgebra de Lie $\mathbb{R}^{n}$ de $\mathbb{R}^{n}$, isto é, um campo constante. Dada uma carta local $(U, \varphi)$ em $\mathbb{R}^{n}$, podemos escrever para todo $p \in U$

$$
X(p)=\sum_{i=1}^{n} f_{i}(p) \frac{\partial}{\partial x_{i}}(p),
$$

e

$$
Y(p)=\sum_{i=1}^{n} g_{i}(p) \frac{\partial}{\partial x_{i}}(p) .
$$


O colchete de Lie $[X, Y] \in \mathbb{R}^{n}$ se $[X, Y]$ é um campo constante em $\mathbb{R}^{n}$. O colchete $[X, Y]$ pode ser escrito como

$$
[X, Y](p)=\sum_{i=1}^{n} k_{i}(p) \frac{\partial}{\partial x_{i}}(p)
$$

onde $k_{i}(p)=\sum_{j=1}^{n} g_{j}(p) \frac{\partial f_{j}}{\partial x_{i}}(p)$. Segue então que esse colchete é um campo constante se toda $k_{i}$ é constante. Mas daí resulta que cada $f_{i}$ é linear, ou seja, $f_{i}(p)=\sum_{i=1}^{n} a_{i j} p_{j}+b_{j}$. Por conseguinte, o campo $X$ é um campo linear tal que $X(p)=A(p)+b$ para um $A \in \mathrm{M}_{n}(\mathbb{R})$ dado por $A=\left(a_{i j}\right)_{1 \leq i, j \leq n}$ e $b=\left(b_{i}\right) \in \mathbb{R}^{n}$.

Observe que no caso Euclideano tem-se: $A_{t}=e^{t A} \in \mathrm{Gl}(n, \mathbb{R})=\operatorname{Aut}\left(\mathbb{R}^{n}\right)$. Portanto, é natural pensarmos a esta altura num campo $X$ em grupo de Lie $G$ tal que seu fluxo $X_{t}$ para todo $t \in \mathbb{R}$ seja um subgrupo a 1-parâmetro do grupo $\operatorname{Aut}(G)$ de automorfismos de $G$. Portanto, a transição do caso linear em $\mathbb{R}^{n}$ para um grupo de Lie $G$ é feito através de introdução de sistemas com normalizador.

Campos lineares em grupos de Lie são nada mais nada menos que automorfismos infinitesimais na literatura de grupos de Lie. São uma extensão natural aos grupos de Lie de campos lineares em espaços vetoriais e por este motivo também são chamados de linear.

Definição 4.1.3. Um campo $X$ sobre um grupo de Lie $G$ é dito um automorfismo infinitesimal se seu fluxo $\left(X_{t}\right)_{t \in \mathbb{R}}$ induzido por $X$ é um subgrupo a 1-parâmetro de $\operatorname{Aut}(G)$.

Os automorfismos infinitesimais foram considerados no contexto da Teoria de controle primeiro por L. Markus [15] em grupos de matrizes (e, posteriormente, no caso geral por Ayala e Tirao em [3]). Mais precisamente, um sistema linear em $\mathbb{R}^{n}$ é um caso particular de um sistema linear sobre grupos de matrizes pois $\mathbb{R}^{n}$ pode ser identificado com um subgrupo $G \subset \operatorname{Gl}(n+1, \mathbb{R})$ através de aplicação

$$
x \longrightarrow P=\left(\begin{array}{ll}
1 & x \\
0 & 1
\end{array}\right) \text {. }
$$

Seja $\Sigma$ o sistema linear $\dot{x}=A x+B u$ em $\mathbb{R}^{n}$. Denote por $b_{1}, \ldots, b_{m}$ as colunas da matriz $B \in M_{n \times m}(\mathbb{R})$. Pode-se definir então em virtude da aplicação acima as matrizes

$$
Y^{j}=\left(\begin{array}{cc}
1 & b_{j} \\
0 & 1
\end{array}\right) \quad \text { e } \quad X=\left(\begin{array}{cc}
A & 0 \\
0 & 0
\end{array}\right)
$$

e, por conseguinte, o sistema $\Sigma$ pode ser representado por

$$
\dot{P}=X P-P X+\sum_{j=1}^{m} u_{j} Y^{j} P
$$


onde $X^{*}(P)=X P-P X$ define o campo $X^{*}$ induzido por um elemento $A$ na álgebra de Lie $M_{n}(\mathbb{R})$ de todas as matrizes $n \times n$ com entradas reais. Neste caso, o fluxo de $X^{*}$ para todo $t \in \mathbb{R}$ é dado por

$$
X_{t}^{*}(P)=e^{t A} \cdot P \cdot e^{-t A}
$$

que é um automorfismo interno (e, portanto, automorfismo) de $G$.

Há uma outra maneira equivalente de definir um campo linear em um grupo de Lie conexo. Veja a definição a seguir.

Definição 4.1.4. Seja $G$ um grupo de Lie conexo cuja álgebra de Lie $\mathfrak{g}$ é identificada com a álgebra de Lie de todos os campos invariantes à esquerda em $G$. Um campo $X$ em $G$ é dito linear se:

$$
\forall Y \in \mathfrak{g} \quad[X, Y] \in \mathfrak{g} .
$$

O normalizador de álgebra de Lie $\mathfrak{g}$ de $G$ na álgebra de Lie $X(G)$ de todos os campos diferenciáveis em $G$ será denotado por $\mathfrak{n}_{X(G)}(\mathfrak{g})$ e é definido como:

$$
\mathfrak{n}_{X(G)}(\mathfrak{g})=\{X \in X(G):[X, Y] \in \mathfrak{g} \text { para todo } Y \in \mathfrak{g}\} .
$$

Os elementos em $\mathfrak{n}_{X(G)}(\mathfrak{g})$ são denominados campos lineares em $G$. A identidade de Jacobi implica que $\mathfrak{n}_{X(G)}(\mathfrak{g})$ é uma subálgebra de $X(G)$, e que a aplicação

$$
\mathfrak{n}_{X(G)}(\mathfrak{g}) \longrightarrow \mathfrak{D e r}(\mathfrak{g}), \quad X \longmapsto \operatorname{ad}_{\mathfrak{g}}(X),
$$

é um homomorfismo de álgebras de Lie.

Uma maneira para produzir automorfismo infinitesimal é usar os automorfismos internos, ou seja, conjugação. Mais precisamente, a ideia que vale na verdade para um grupo arbitrário não comutativo é a seguinte: tome um campo $Z \in \mathfrak{g}$ e defina

$$
X_{t}(x)=\exp (t Z) \cdot x \cdot \exp (-t Z), \quad x \in G, \quad t \in \mathbb{R} .
$$

Desta forma, $X_{t}$ do jeito que está definido torna-se um automorfismo infinitesimal de $G$ para todo $t \in \mathbb{R}$ cuja inversa é $\left(X_{t}\right)^{-1}=X_{-t}$. Segue que o campo $X$ num ponto $x \in G$ é por definição

$$
X(x)=\left(\frac{d}{d t}\right)_{t=0} X_{t}(x)=\left(\frac{d}{d t}\right)_{t=0} \exp (t Z) \cdot x \cdot \exp (-t Z) .
$$

Exemplo 4.1.5. Seja $G$ o grupo de Heisenberg de dimensão 3 e sejam

$$
x=\left(\begin{array}{ccc}
1 & x & y \\
0 & 1 & z \\
0 & 0 & 1
\end{array}\right) \in G \quad \text { e } \quad Y^{1}=\left(\begin{array}{ccc}
0 & 1 & 0 \\
0 & 0 & 0 \\
0 & 0 & 0
\end{array}\right) \in \mathfrak{g} .
$$


Calculando as exponenciais obtemos:

$$
\exp \left(\mp t Y^{1}\right)=\left(\begin{array}{ccc}
1 & \mp t & 0 \\
0 & 1 & 0 \\
0 & 0 & 1
\end{array}\right)
$$

Então

$$
\begin{aligned}
& X_{t}(x)=\exp \left(t Y^{1}\right) \cdot x \cdot \exp \left(-t Y^{1}\right) \\
& X_{t}(x)=\left(\begin{array}{lll}
1 & t & 0 \\
0 & 1 & 0 \\
0 & 0 & 1
\end{array}\right)\left(\begin{array}{lll}
1 & x & y \\
0 & 1 & z \\
0 & 0 & 1
\end{array}\right)\left(\begin{array}{ccc}
1 & -t & 0 \\
0 & 1 & 0 \\
0 & 0 & 1
\end{array}\right)
\end{aligned}
$$

e, portanto,

$$
X(x)=\left(\frac{d}{d t}\right)_{t=0} X_{t}(x)=\left(\begin{array}{ccc}
0 & 0 & z \\
0 & 0 & 0 \\
0 & 0 & 0
\end{array}\right)=z Y^{3}
$$

A seguir define-se formalmente um sistema linear num grupo de Lie.

Definição 4.1.6. Um sistema linear $\Sigma$ em um grupo de Lie conexo $G$ de dimensão finita é dado pela seguinte informação

$$
\Sigma=(G, \mathcal{D})
$$

onde $\mathcal{D}$ denota a dinâmica dada pela família de equações diferenciais ordinárias em $G$

$$
\dot{x}(t)=X(x)+\sum_{j=1}^{m} u_{j} Y^{j}(x), \quad x \in G,
$$

parametrizadas por $u \in \mathcal{U}_{m}$.

O campo vetorial $X$ em (4.2) livre de controle é chamado de campo drift que por sua vez pertence ao normalizador $\mathfrak{n}_{X(G)}(\mathfrak{g})$ de $\mathfrak{g}$ em $X(G)$ enquanto que os campos sujeitos a controle $Y^{j}, j=1,2, \ldots, m$, são elementos de álgebra de Lie $\mathfrak{g}$ de campos de vetores invariantes à esquerda. As funções de entrada $u=\left(u_{1}, u_{2}, \ldots, u_{m}\right)$ pertencem a classe $\mathcal{U}_{m}$ de controles admissíveis e irrestritos. Mais precisamente, os elemenstos de $\mathcal{U}_{m}$ são funções constantes por pedaços da forma $u:[0, \infty) \rightarrow \mathbb{R}^{m}$.

A dinâmica $\mathcal{D}$ associada ao sistema $\Sigma$ é dada pela seguinte coleção

$$
\mathcal{D}=\left\{X+\sum_{j=1}^{m} u_{j} Y^{j}: u \in \mathbb{R}^{m}\right\}
$$




\subsection{O Normalizador de $\mathfrak{g}$ em $X(G)$}

Nesta subseção veremos a caracterização do normalizador $\mathfrak{n}_{X(G)}(\mathfrak{g})$ considerando uma identificação útil de campos com funções diferenciáveis em $G$ a valores em sua álgebra de Lie g. Tal caracterização ajudará para obtenção de soluções de sistemas lineares em grupos de Lie.

Mas primeiro provaremos que dado um grupo de Lie $G$ conexo o centralizador $\mathfrak{z}_{X(G)}(\mathfrak{g})$ de sua álgebra de Lie em $X(G)$ consiste de todos os campos invariantes à direita em $G$.

Proposição 4.2.1. Seja $G$ um grupo de Lie conexo com álgebra de Lie g. O núcleo da aplicação $X \rightarrow \operatorname{ad}_{\mathfrak{g}}(X)$ de normalizador $\mathfrak{n}_{X(G)}(\mathfrak{g})$ em $\mathfrak{D e r}(\mathfrak{g})$ é o centralizador $\mathfrak{z} X(G)(\mathfrak{g})$ de $\mathfrak{g}$ em $X(G)$, que por sua vez consiste de todos os campos invariantes à direita em $G$.

Demonstração: Pelo Lema 1.4.12, sabe-se que dois campos vetorias comutam se, e somente, se seus fluxos locais comutam. Como o fluxo local de um campo $Z \in \mathfrak{g}$ é dado por $Z_{t}(g)=g \exp (t Z)$, obtemos:

$$
Y \in \mathfrak{z} X(G)(\mathfrak{g}) \Longleftrightarrow Y_{s}\left(Z_{t}(g)\right)=Z_{t}\left(Y_{s}(g)\right),
$$

para todo $Z \in \mathfrak{g}, g \in G$, e $s, t \in \mathbb{R}$. Segue então que para um grupo de Lie conexo temse: $Y_{s}(g h)=Y_{s}(g) h$ para todo $h \in G$ uma vez que qualquer $h \in G$ é, pelo Corolário 2.5.8 um produto finito de exponenciais. Mas, isto é equivalente ao dizer que o fluxo local de um campo $Y$ é invariante à direita. De fato, se $Y_{s}(g h)=Y_{s}(g) h$ para todo $h \in G$ então

$$
Y(g h)=\left(\frac{d}{d t}\right)_{t=0} Y_{t}(g h)=\left(\frac{d}{d t}\right)_{t=0} Y_{t}(g) h=\left(d R_{h}\right)_{g} Y(g),
$$

isto é, $Y$ é um campo invariante à direita. Reciprocamente, se $Y$ é um campo invariante à direita então

$$
\frac{d}{d t} Y_{t}(g) h=\left(d R_{h}\right)_{Y_{t}(g)} Y\left(Y_{t}(g)\right)=Y\left(Y_{t}(g) h\right)
$$

e

$$
Y_{0}(g) h=g h=Y_{0}(g h) .
$$

Daí, $Y_{t}(g) h$ e $Y_{t}(g h)$ ambas são as soluções de uma mesma equação diferencial ordinária de primeira ordem e que coincidem em $t=0 \mathrm{e}$, portanto, são iguais. Com isso terminase a prova de que para um grupo conexo o centralizador $\mathfrak{z}_{X(G)}(\mathfrak{g})$ consiste de todos os campos invariantes à direita. 
Uma demonstração alternativa da Proposição acima foi recentemente apresentada por P. Jouan em [9].

Por questão de simplicidade, a partir de agora adotaremos as notações $\mathfrak{n}(\mathfrak{g})$ e $\mathfrak{z}(\mathfrak{g})$ em vez de $\mathfrak{n}_{X(G)}(\mathfrak{g})$ e $\mathfrak{z} X(G)(\mathfrak{g})$, respectivamente, desde que a álgebra de Lie em que $\mathfrak{g}$ aperece como um subconjunto é $X(G)$, veja Definições 2.3.4 e 2.3.5.

Proposição 4.2.2. Seja $\mathfrak{h}=\{X \in \mathfrak{n}(\mathfrak{g}): X(e)=0\}$. Então hy é uma subálgebra de $\mathfrak{n}(\mathfrak{g})$.

Demonstração: Que h é um subespaço vetorial é imediato. Agora, dados $X^{1}, X^{2} \in \mathfrak{h}$ tem-se: $\left[X^{1}, X^{2}\right](e)=0$ se, e somente, se a curva dada em (1.13) satisfaz

$$
\alpha(t)=e=\left(X_{-t}^{2} \circ X_{-t}^{1} \circ X_{t}^{2} \circ X_{t}^{1}\right)(e) .
$$

Mas isso segue justamente pela hipótese de que $X^{1}$ e $X^{2}$ são campos lineares com singularidade no elemento neutro $e \in G$.

No que segue prova-se que $\mathfrak{z}(\mathfrak{g}) \otimes \mathfrak{h} \cong \mathfrak{n}(\mathfrak{g})$ representa o normalizador $\mathfrak{n}(\mathfrak{g})$ como produto semidireto de $\mathfrak{h}$ e $\mathfrak{z}(\mathfrak{g})$ e, que $X \mapsto \operatorname{ad}_{\mathfrak{g}}(X)$ é um isomorfismo de álgebra de Lie de $\mathfrak{h}$ sobre a imagem de $\mathfrak{n}(\mathfrak{g})$ em $\mathfrak{D} \mathfrak{e r}(\mathfrak{g})$.

Observando que $\mathfrak{z}(\mathfrak{g})$ é um ideal de $\mathfrak{n}(\mathfrak{g})$ pode-se concluir que $[\mathfrak{z}(\mathfrak{g}), \mathfrak{h}] \subset \mathfrak{z}(\mathfrak{g})$. Por outro lado, como um campo invariante à direita que se anula em um ponto é o campo nulo segue que $\mathfrak{z}(\mathfrak{g}) \cap \mathfrak{h}=\{0\}$ e, portanto, podemos de fato formar o produto semidireto $\mathfrak{z}(\mathfrak{g}) \otimes \mathfrak{h}$.

Dado $X \in \mathfrak{n}(\mathfrak{g})$, seja $Y$ o campo invariante à direita em $G$ tal que $Y(e)=X(e)$. Então, $X-Y \in \mathfrak{h}$, e $\operatorname{ad}_{\mathfrak{g}}(X)=\operatorname{ad}_{\mathfrak{g}}(X-Y)$ já que $Y \in \mathfrak{z}(\mathfrak{g})$, o núcleo da aplicação $X \mapsto \operatorname{ad}_{\mathfrak{g}}(X)$. Segue que $\mathfrak{n}(\mathfrak{g})=\mathfrak{z}(\mathfrak{g}) \oplus \mathfrak{h}$ e que $X \mapsto \operatorname{ad}_{\mathfrak{g}}(X): \mathfrak{h} \rightarrow \mathfrak{D e r}(\mathfrak{g})$ é um isomorfismo de álgebra de Lie sobre a imagem de $\mathfrak{n}(\mathfrak{g})$ em $\mathfrak{D} \mathfrak{e r}(\mathfrak{g})$. Daí resulta que a aplicação

$$
\mathfrak{z}(\mathfrak{g}) \otimes \mathfrak{h} \longrightarrow \mathfrak{n}(\mathfrak{g}), \quad(Z, Y) \longmapsto Z+Y,
$$

é um isomorfismo de álgebra de Lie. Se $Z$ é um campo invariante à direita em $G$ então existe pelo Lema 2.4.6 um único campo invariante à esquerda $Z^{L}$ em $G$ tal que $Z^{L}(e)=-Z(e)$. Daí, a aplicação linear

$$
\mathfrak{z}(\mathfrak{g}) \longrightarrow \mathfrak{g}, \quad Z \longmapsto Z^{L}
$$

é um isomorfismo de álgebra de Lie de $\mathfrak{z}(\mathfrak{g})$ sobre $\mathfrak{g}$.

Dados $Z \in \mathfrak{z}(\mathfrak{g})$ e $X \in \mathfrak{h}$ considere a aplicação abaixo

$$
\Phi: \mathfrak{n}(\mathfrak{g}) \longrightarrow \mathfrak{g} \otimes \mathfrak{D e r}(\mathfrak{g}), \quad Z+X \longmapsto\left(Z^{L}, \operatorname{ad}_{\mathfrak{g}}(X)\right) .
$$

Temos provado então a primeira parte do seguinte resultado: 
Teorema 4.2.3. (Teorema 2.2, [3]) (i) Seja G um grupo de Lie conexo com álgebra de Lie $\mathfrak{g}$. Então a aplicação $\mathfrak{z}(\mathfrak{g}) \otimes \mathfrak{h} \rightarrow \mathfrak{n}(\mathfrak{g})$ dada por $(Z, Y) \mapsto Z+Y$ é um isomorfismo de álgebra de Lie enquanto que a aplicação $\Phi: \mathfrak{n}(\mathfrak{g}) \rightarrow \mathfrak{g} \otimes \mathfrak{D e r}(\mathfrak{g})$ definida por $\Phi(Z+X)=$ $\left(Z^{L}, \operatorname{ad}_{\mathfrak{g}}(X)\right)$ para todo $Z \in \mathfrak{z}(\mathfrak{g})$ e $X \in \mathfrak{h}$ é um homomorfismo injetora de álgebras de Lie (ou seja, isomorfismo sobre sua imagem).

(ii) Se $G$ um grupo de Lie conexo e simplesmente conexo com álgebra de Lie $\mathfrak{g}$. Então a aplicação $\Phi: \mathfrak{n}(\mathfrak{g}) \rightarrow \mathfrak{g} \otimes \mathfrak{D e r}(\mathfrak{g})$ é sobrejetora e, portanto, é um isomorfismo de álgebra de Lie.

Demonstração: (ii). Dado $D \in \mathfrak{D} \mathfrak{e r}(\mathfrak{g})$, então $\exp (t D)$ é um automorfismo de $\mathfrak{g}$ para todo $t \in \mathbb{R}$. Como $G$ é conexo e simplesmente conexo então existe um único automorfismo $\theta_{t}$ de $G$ tal que $\left(d \theta_{t}\right)_{e}=\exp (t D)$ e, por conseguinte, obtem-se um fluxo diferenciável $(g, t) \rightarrow \theta_{t}(g)$ em $G$. Seja $X$ o campo associado a esse fluxo e vamos provar que $X$ pertence ao normalizador $\mathfrak{n}(\mathfrak{g})$ de $\mathfrak{g}$. Mais precisamente, se $Y \in \mathfrak{g}$ (isto é, $Y$ é um campo invariante à esquerda em $\mathfrak{g}$ ) então segue pelas equações dadas em (1.14) e (1.15) que

$$
L_{X}(Y)(g)=[X, Y](g)=\left(\frac{d}{d t}\right)_{t=0} d\left(\theta_{-t}\right) Y\left(\theta_{t}(g)\right)=\left(\frac{d}{d t}\right)_{t=0} d\left(\theta_{-t}\right) d L_{\theta_{t}(g)} Y(e) .
$$

Como foi visto na seção de homomorfismos, a equação (2.3) vale para todo homomorfismo de Lie $G \rightarrow H$. Substituindo nessa equação $g$ por $\theta_{t}(g)$ e $\phi$ por $\theta_{-t}$ vale a igualdade

$$
\theta_{-t} \circ L_{\theta_{t}(g)}=L_{\theta_{-t}\left(\theta_{t}(g)\right)} \circ \theta_{-t}
$$

e, portanto,

$$
d\left(\theta_{-t} \circ L_{\theta_{t}(g)}\right)=d\left(L_{g} \circ \theta_{-t}\right) .
$$

Daí obtemos a nossa última equação em (4.3) como:

$$
\begin{aligned}
& =\left(\frac{d}{d t}\right)_{t=0}\left(d L_{g} \circ d\left(\theta_{-t}\right)\right) Y(e) \\
& =d L_{g}\left(\frac{d}{d t}\right)_{t=0} d\left(\theta_{-t}\right) Y(e)=d L_{g}\left(\frac{d}{d t}\right)_{t=0} \exp (-t D) Y(e)=-D(Y)(g) .
\end{aligned}
$$

Portanto, $X \in \mathfrak{n}(\mathfrak{g})$ e $\operatorname{ad}_{\mathfrak{g}}(X)=-D$. Mais ainda, $X(e)=\left(\frac{d}{d t}\right)_{t=0} \theta_{t}(e)=0$, ou seja, $X$ possui singularidade em $e \in G$. Isto termina a prova.

\subsection{Automorfismos de Grupos e Álgebras de Lie}

Os grupos de automorfismos dos grupos de Lie são estudados através dos grupos de automorfismos de suas álgebras de Lie. Se $\tau$ é um automorfismo de um grupo de 
Lie $G$ então pela Proposição 2.3.8 sua diferencial na origem $d \tau_{e}$ é um automorfismo da álgebra de Lie $\mathfrak{g}$ de $G$. Isso define a aplicação

$$
\delta: \operatorname{Aut}(G) \longrightarrow \operatorname{Aut}(\mathfrak{g}), \quad \delta(\tau)=d \tau_{e}
$$

Pela regra da cadeia essa aplicação é um homomorfismo diferenciável de grupos.

Proposição 4.3.1. Se $G$ é conexo então $\delta$ é injetora.

Demonstração: Veja Proposição 6.1 de [22].

Quanto a sobrejetividade de $\delta$ pode-se usar o Teorema 2.3.9, que garante que todo automorfismo de $\mathfrak{g}$ se estende a um automorfismo de $G$ desde que o grupo seja simplesmente conexo.

Proposição 4.3.2. Se $G$ é conexo e simplesmente conexo então $\delta$ é sobrejetora.

Estas duas Proposições juntas fornecem o grupo de automorfismos de um grupo de Lie $G$ simplesmente conexo.

Proposição 4.3.3. Se $G$ é conexo e simplesmente conexo então $\operatorname{Aut}(G)$ é isomorfo a $\operatorname{Aut}(\mathfrak{g})$. Um isomorfismo é dado pela aplicação em (4.4).

Demonstração: Veja prova da Proposição 6.3 em [22].

Em geral o grupo de automorfismos $\operatorname{Aut}(G)$ de um grupo de Lie $G$ pode ser bem diferente do grupo de automorfismos $\operatorname{Aut}(\mathfrak{g})$ de sua álgebra de Lie $\mathfrak{g}$. De qualquer forma, no caso em que $G$ é conexo o grupo $\operatorname{Aut}(G)$ é isomorfo à imagem de $\delta$ já que $\delta$ é injetora. Em outras palavras, $\operatorname{Aut}(G)$ se identifica a um subgrupo de $\operatorname{Aut}(\mathfrak{g})$, que por sua vez, é isomorfo a $\operatorname{Aut}(\widetilde{G})$, onde $\widetilde{G}$ é o recobrimento simplesmente conexo de $G$. Isto é, $\operatorname{Aut}(G)$ se identifica a um subgrupo de $\operatorname{Aut}(\widetilde{G})$. Esse subgrupo é a imagem do homomorfismo

$$
\widetilde{\delta}: \operatorname{Aut}(G) \longrightarrow \operatorname{Aut}(\widetilde{G}), \quad \widetilde{\delta}(\tau)=\widetilde{\tau}
$$

onde $\widetilde{\tau} \in \operatorname{Aut}(\widetilde{G})$ é o único automorfismo associado que satisfaz $d \widetilde{\tau}_{e}=d \tau_{e}$.

Para descrever a imagem desse homomorfismo, seja $D \subset \widetilde{G}$ o subgrupo discreto central tal que $G=\widetilde{G} / D$ e denote por $\pi: \widetilde{G} \rightarrow G=\widetilde{G} / D$ a projeção canônica. Essa projeção satisfaz $d \pi_{e}=\left.\mathrm{id}\right|_{\mathfrak{g}}$ (já que as álgebras de Lie de $G$ e $\widetilde{G}$ são identificadas entre si). Agora, seja

$$
\operatorname{Aut}_{D}(\widetilde{G})=\{\sigma \in \operatorname{Aut}(\widetilde{G}): \sigma(D)=D\} \subset \operatorname{Aut}(\widetilde{G})
$$

o subgrupo que deixa invariante o núcleo de $\pi$. 
Proposição 4.3.4. Seja $G$ um grupo de Lie conexo. Então Aut $(G)$ é isomorfo a $\operatorname{Aut}_{D}(\widetilde{G})$ onde $G=\widetilde{G} / D$. O isomorfismo é dado por $\widetilde{\delta}: \tau \rightarrow \widetilde{\tau}$ onde $\tau \in \operatorname{Aut}(G)$ e $\widetilde{\tau}$ é o único automorfismo de $\widetilde{G}$ tal que $d \widetilde{\tau}_{e}=d \tau_{e}$.

Como corolário dessa Proposição, concula-se que se $G$ é conexo então $\operatorname{Aut}(G)$ é grupo de Lie e sua ação em $G$ é diferenciável.

A questão agora é descrever a álgebra de Lie de $\operatorname{Aut}(G)$ (para $G$ conexo), que é denotado por aut $(G)$. Foi mencionado acima que se $G$ é simplesmente conexo então $\mathfrak{a} \mathfrak{u t}(G)$ é isomorfo à álgebra das derivações $\mathfrak{D} \mathfrak{e r}(\mathfrak{g})$. Por esse isomorfismo $\mathfrak{a} \mathfrak{u}(G)$ é vista como uma álgebra de Lie de transformações lineares de $\mathfrak{g}$. Em suma, o grupo de Lie $\operatorname{Aut}(G)$ de um grupo de Lie conexo $G$ é um subgrupo de $\operatorname{Aut}(\widetilde{G})$ do recobrimento universal de $G$. Dessa forma $\mathfrak{a u t}(G)$ é uma subálgebra de $\mathfrak{a u t}(\widetilde{G})$. Através dos automorfismos infinitesimais é fácil identificar essa subálgebra. Mais precisamente, se $G=\widetilde{G} / D$ então $\operatorname{Aut}(G)$ é o subgrupo dos elementos que deixam $D$ invariante. Daí que

$$
\mathfrak{a u t}(G)=\{Z \in \mathfrak{a u t}(\widetilde{G}): Z(x)=0, \forall x \in D\} \subset \mathfrak{a u t}(\widetilde{G}) .
$$

Seja $G$ um grupo de Lie conexo e simplesmente conexo. Sabe-se que os grupos de Lie $\operatorname{Aut}(G)$ e $\operatorname{Aut}(\mathfrak{g})$ são isomorfos via aplicação $\delta$ dada em (4.4) e, que o grupo Aut( $\mathfrak{g})$ possui uma única estrutura de grupo de Lie (por ser um subgrupo fechado do grupo linear $\operatorname{Gl}(\mathfrak{g}))$ cuja álgebra de Lie é $\mathfrak{D} \mathfrak{e r}(\mathfrak{g})$. Em virtude disso, a álgebra de Lie $\mathfrak{a} \mathfrak{u}(G)$ do grupo $\operatorname{Aut}(G)$ identifica-se com a do grupo $\operatorname{Aut}(\mathfrak{g})$, ou seja, $\mathfrak{D e r}(\mathfrak{g})$. Denote por $\operatorname{Exp}: \operatorname{Der}(\mathfrak{g}) \rightarrow \operatorname{Aut}(G)$ a aplicação exponencial correspondente.

Agora, seja $\pi: \widetilde{G} \rightarrow G$ o homomorfismo de recobrimento universal de um grupo de Lie conexo $G$ e seja $K=\operatorname{ker} \pi$. Então o grupo Aut $(G)$ se identifica com o subgrupo $\{\sigma \in \operatorname{Aut}(\widetilde{G}): \sigma(K) \subseteq K\}$ de $\operatorname{Aut}(\widetilde{G})$ enquanto sua álgebra de Lie $\mathfrak{a u t}(G)$ se identifica com uma subálgebra de Lie de $\mathfrak{D e r}(\mathfrak{g})$. Também denotaremos por Exp : aut $(G) \rightarrow$ $\operatorname{Aut}(G)$ a aplicação exponencial correspondente. Da diagrama

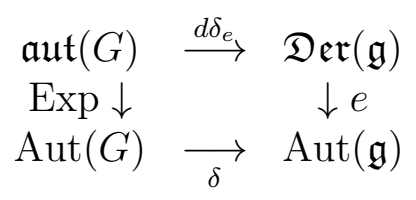

onde $e$ denota a aplicação exponencial entre $\mathfrak{D} \mathfrak{e r}(\mathfrak{g})$ e $\operatorname{Aut}(\mathfrak{g})$, observa-se que para todo $D \in \mathfrak{a u t}(G)$ vale:

$$
(\delta \circ \operatorname{Exp})(D)=\left(e \circ d \delta_{e}\right)(D)
$$

e, portanto,

$$
d(\operatorname{Exp} D)_{e}=e^{D}
$$

uma vez que $\delta(\operatorname{Exp} D)=d(\operatorname{Exp} D)_{e}$ e $d \delta_{e}(D)=D$. 
Por outro lado, $\operatorname{como} \operatorname{Exp} D \in \operatorname{Aut}(G)$ dado um $X \in \mathfrak{g}$ segue da diagrama

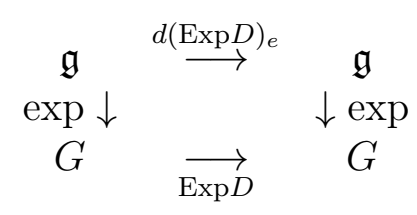

que obtemos

$$
\operatorname{Exp} D \exp X=\exp \left(d(\operatorname{Exp} D)_{e} X\right)=\exp \left(e^{D}(X)\right) .
$$

Seja $\varsigma: X(G) \rightarrow X(\widetilde{G})$ a aplicação que a um campo $X \in X(G)$ associa o único campo $\widetilde{X}$ em $\widetilde{G}$, que é $\pi$-relacionado com $X$, isto é, $d \pi(\widetilde{X}(g))=X(\pi(g))$, para todo $g \in \widetilde{G}$ (Veja Definição 1.4.10).

Pela invariancia de colchete de Lie temos a inclusão

$$
\widetilde{i}: \mathfrak{n}_{X(G)}(\mathfrak{g}) \hookrightarrow \mathfrak{n}_{X(\widetilde{G})}(\mathfrak{g}) .
$$

Teorema 4.3.5. Seja $\pi: \widetilde{G} \rightarrow G$ o homomorfismo de recobrimento universal de um grupo de Lie conexo $G$. Então a aplicação $\Phi$ manda $\mathfrak{n}_{X(G)}(\mathfrak{g})$ em $\mathfrak{g} \otimes \mathfrak{a u t}(G)$ isomorficamente. Mais ainda, se $i: \mathfrak{g} \otimes \mathfrak{a u t}(G) \hookrightarrow \mathfrak{g} \otimes \mathfrak{D e r}(\mathfrak{g})$ denota a inclusão então o diagrama

$$
\begin{array}{cccc}
\mathfrak{n}_{X(\widetilde{G})}(\mathfrak{g}) & \stackrel{\Phi}{\longrightarrow} & \mathfrak{g} \otimes \mathfrak{D e r}(\mathfrak{g}) \\
\underset{i \uparrow}{ } & & \uparrow i \\
\mathfrak{n}_{X(G)}(\mathfrak{g}) & \longrightarrow & \mathfrak{g} \otimes \mathfrak{a u t}(G)
\end{array}
$$

comuta.

Demonstração: Pela invariancia de colchete de Lie pode-se ver commutatividade de diagrama a seguir:

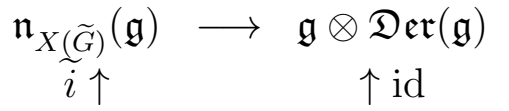

$$
\begin{aligned}
& \mathfrak{n}_{X(G)}(\mathfrak{g}) \longrightarrow \mathfrak{g} \otimes \mathfrak{D e r}(\mathfrak{g})
\end{aligned}
$$

Portanto, a única que coisa que resta pra provarmos é que $\Phi(\mathfrak{h})=\mathfrak{a u t}(G)$. Dado $X \in X(G)$, sejam $\widetilde{X}_{t}$ e $X_{s}$ os fluxos dos campos $\varsigma(X)=\widetilde{X}$ e $X$, respectivamente. Então

$$
\pi\left(\widetilde{X}_{t}(g)=X_{t}(\pi(g)),\right.
$$

para todo $g \in \widetilde{G}$ e $t \in \mathbb{R}$. Agora, se $X \in \mathfrak{h}$ e $D=\Phi(\varsigma(X))$ então

$$
\pi(\operatorname{Exp}(-t D)(g))=\pi\left(\widetilde{X}_{t}(g)\right)=X_{t}(\pi(g)) .
$$

Em particular, se colocarmos $g=k \in K=\operatorname{ker} \pi$ a última equação torna-se:

$$
\pi(\operatorname{Exp}(-t D)(k))=X_{t}(e),
$$


uma vez que $X$ tem singularidade em $e$, isto é, $X(e)=0$. Daí segue que $\operatorname{Exp}(t D)(K) \subset$ $K$ o que por sua vez implica que $D \in \mathfrak{a u t}(G)$.

De exatamente mesma maneira, pode-se provar que se $D \in \mathfrak{a u t}(G)$ então o campo vetorial $\widetilde{X} \in \mathfrak{n}_{X(\widetilde{G})}(\mathfrak{g})$ tal que $\Phi(\widetilde{X})=D$ (Teorema 4.2.3) é $\pi$-relacionado a um único campo $X \in \mathfrak{h}$.

\subsection{Identificação de Campos com Funções}

A seguir falamos um pouco sobre como identificar campos vetoriais em $G$ com funções diferenciáveis de $G$ em $T_{e} G$. Mais precisamente, um campo $X \in X(G)$ induz uma função $F: G \rightarrow \mathfrak{g}$ definida por

$$
F(x)=d L_{x^{-1}} X(x), \quad x \in G .
$$

Reciprocamente, tal função também dá origem a um campo $X$ definido por

$$
X(x)=d L_{x} F(x), \quad x \in G .
$$

Por questão de simplicidade, dado $F: G \rightarrow \mathfrak{g}$ denotaremos por $\widetilde{F}$ o campo invariante à esquerda dado por

$$
\widetilde{F}_{x}=d L_{x} F(x), \quad x \in G,
$$

tal que $\widetilde{F}_{e}=F$.

Além de $\widetilde{F}_{x}$ usaremos de vez em quando uma outra notação $\widetilde{F(x)}$ para denotar o mesmo campo induzido por $F$. Isso porque quem gera um campo em $\mathfrak{g}$ é o $F(x)$ por algum $x \in G$. Então fica razoável também a adoção da notação $\widetilde{F(x)})_{x}$.

Existe uma apresentação em [3] baseada nesse meio de identificar os campos em $G$ com as funções diferenciáveis de $G$ a valores na álgebra de Lie $\mathfrak{g}$. A razão para isso é o resultado obtido em Teorema 4.4.3. Mas antes disso achamos conveniente começar com a seguinte

Proposição 4.4.1. A aplicação $C^{\infty}(G, \mathfrak{g}) \rightarrow X(G)$ dada por $F \rightarrow \widetilde{F}$ é um isomorfismo de Lie.

Demonstração: $\operatorname{Sejam~} F, H \in C^{\infty}(G, \mathfrak{g})$ e $c_{1}, c_{2} \in \mathbb{R}$. Então

$$
\begin{aligned}
\left(c_{1} \widetilde{F+c_{2}} H\right)_{x} & =d L_{x}\left(c_{1} F+c_{2} H\right)(x)=c_{1} d L_{x} F(x)+c_{2} d L_{x} H(x) \\
& =c_{1} \widetilde{F}_{x}+c_{2} \widetilde{H}_{x}
\end{aligned}
$$

ou seja, a aplicação é linear. Agora, seja $\widetilde{F}=\widetilde{H}$, isto é, $\widetilde{F}_{x}=\widetilde{H}_{x}$ para todo $x \in G$. Então

$$
\widetilde{F}_{x}=d L_{x} F(x)=d L_{x} H(x)=\widetilde{H}_{x}
$$


implica para todo $x \in G$ que $F(x)=H(x)$ já que $d L_{x}$ é um isomorfismo entre espaços vetoriais com a inversa $\left(d L_{x}\right)^{-1}=d L_{x^{-1}}$. Daí segue que $F=H$, ou seja, a correspondência no enunciado da Proposição é, de fato, injetora. A sobrejetividade é imediato a partir de equação em ( 4.9). Ou seja, sempre que for dado um campo $X$ em $G$ tem-se a função correspondente $F$ de tal forma que $\widetilde{F}=X$.

Note-se que as funções constantes correspondem sob esse isomorfismo às campos invariantes à esquerda.

Lema 4.4.2. (A função associada ao colchete) Dados $Y \in \mathfrak{g}$ e $F \in C^{\infty}(G, \mathfrak{g})$, seja $H \in C^{\infty}(G, \mathfrak{g})$ definida por

$$
H(x)=\widetilde{Y}_{x}(F)+[Y, F(x)], \quad x \in G .
$$

Então, $\widetilde{H}=[\widetilde{Y}, \widetilde{F}]$. Mais ainda, se $\widetilde{F} \in \mathfrak{n}(\mathfrak{g})$ por algum $F \in C^{\infty}(G, \mathfrak{g})$ então $\Phi(\widetilde{F})=$ $\left(-F(e), \operatorname{ad}(F(e))-d F_{e}\right)$.

Demonstração: Observe primeiro que a função $H$ está bem definida pois o lado direito da equação (4.10) pertence mesmo à algebra de Lie $\mathfrak{g}$. De fato, seja $f \in C^{\infty}(G)$. A derivada direcional de $f$ ao longo do campo $F(x) \in \mathfrak{g}$ é dada por

$$
F(x)_{e}(f)=\left(\frac{d}{d t}\right)_{t=0} f(\exp t F(x))
$$

Seja $Y \in \mathfrak{g}$, procedendo da mesma forma tem-se:

$$
\left(\widetilde{Y}_{(.)} F\right)(x)=\tilde{Y}_{x}(F)=\left(\frac{d}{d t}\right)_{t=0} F(x \exp t Y)
$$

Daí segue que $\widetilde{Y}_{x}(F) \in \mathfrak{g}$ pois $\widetilde{Y}_{(.)} F \in C^{\infty}(G, \mathfrak{g})$.

Da forma análoga, segue que $[Y, F().] \in C^{\infty}(G, \mathfrak{g})$ o que implica por sua vez que $\widetilde{Y}_{x}(F)+[Y, F(x)] \in \mathfrak{g}$ cuja função geradora $H \in C^{\infty}(G, \mathfrak{g})$.

Voltando a demonstração. Aplicando a definição do colchete de Lie temos,

$$
[\widetilde{Y}, \widetilde{F}](f)(x)=[\widetilde{Y}, \widetilde{F}]_{x}(f)=(\widetilde{Y}(\widetilde{F} f))(x)-(\widetilde{F}(\widetilde{Y} f))(x)
$$


onde

$$
\begin{aligned}
(\widetilde{Y}(\widetilde{F} f))(x) & =\widetilde{Y}_{x}(\widetilde{F} f) \\
& =\left(\frac{d}{d t}\right)_{t=0}(\widetilde{F} f)(x \exp t Y)=\left(\frac{d}{d t}\right)_{t=0} \widetilde{F}_{x \exp t Y}(f) \\
& =\left(\frac{d}{d t}\right)_{t=0} F(\widetilde{\exp t Y})_{x \exp t Y}(f) \\
& =\left(\frac{d}{d t}\right)_{t=0} F(x \widetilde{\exp t} Y)(f)(x \exp t Y) \\
& =\left(\frac{d}{d t}\right)_{t=0} F(x \widetilde{\exp t Y})(f)(x)+\left(\frac{d}{d t}\right)_{t=0} \widetilde{F(x)}(f)(x \exp t Y) \\
& =(\widetilde{(\widetilde{Y} F)}(x) f)(x)+(\widetilde{Y}(\widetilde{F(x)} f))(x) .
\end{aligned}
$$

Pois

$$
(\widetilde{\widetilde{Y} F)}(x) f)=\left(\frac{d}{d t}\right)_{t=0}(F(\widetilde{\widetilde{\exp t} Y) f})(x)
$$

e

$$
\left(\frac{d}{d t}\right)_{t=0}(\widetilde{F(x)} f)(x \exp t Y)=\tilde{Y}_{x}(\widetilde{F(x)} f)=(\tilde{Y}(\widetilde{F(x)} f))(x) .
$$

Assim,

$$
(\widetilde{Y}(\widetilde{F} f))(x)=(\widetilde{(\widetilde{Y} F)}(x) f)(x)+(\widetilde{Y}(\widetilde{F(x)} f))(x) .
$$

enquanto

$$
(\widetilde{F}(\widetilde{Y} f))(x)=\widetilde{F}_{x}(\widetilde{Y} f)=\widetilde{F(x)_{x}}(\widetilde{Y} f)=\widetilde{F(x)}(\widetilde{Y} f)(x)
$$

Por fim a equação (4.11) se lê como:

$$
\begin{aligned}
{[\widetilde{Y}, \widetilde{F}](f)(x) } & =(\widetilde{\widetilde{Y} F(x)}) f)(x)+(\widetilde{Y}(\widetilde{F(x)} f))(x)-\widetilde{F(x)}(\widetilde{Y} f)(x) \\
& =((\widetilde{\widetilde{Y} F(x)}) f)(x)+([\widetilde{Y} \widetilde{F(x)}] f)(x) \\
& =(\widetilde{\widetilde{Y} F(x)}) f)(x)+([\widetilde{Y, F(x)}] f)(x) .
\end{aligned}
$$

Logo pela linearidade de

$$
\left.\left.\left.[\tilde{Y}, \tilde{F}]_{x}=\widetilde{\left(\tilde{Y}_{x}(F)\right.}\right)_{x}+\widetilde{[Y, F(x)}\right]_{x}=\left(\tilde{Y}_{x}(F) \widetilde{+[Y}, F(x)\right]\right)_{x}=\tilde{H}_{x}
$$

Concluímos aasim que

$$
\tilde{H}=[\tilde{Y}, \tilde{F}]
$$


Agora seja $\widetilde{F}=X+Z$ onde $Z \in \mathfrak{z}(\mathfrak{g})$ e $X \in \mathfrak{h}$. Então $\Phi(\widetilde{F})=\left(Z_{e}, \operatorname{ad}_{\mathfrak{g}}(X)\right)=$ $\left(-F(e), \operatorname{ad}_{\mathfrak{g}}(X)\right.$. Dado $Y \in \mathfrak{g}$ temos

$$
[X, \tilde{Y}]_{e}=[\widetilde{F}, \widetilde{Y}]_{e}=-\widetilde{Y}_{e}(F)-[Y, F(e)]=-d F_{e}(Y)+[F(e), Y]
$$

Portanto, $\operatorname{ad}_{\mathfrak{g}}(X)=\operatorname{ad}(F(e))-d F_{e}$ e consequentemente $d F_{e}$ é uma derivação de $\mathfrak{g}$.

Teorema 4.4.3. (Teorema 2.5, [3]) Se $F \in C^{\infty}(G, \mathfrak{g})$ então $\widetilde{F} \in \mathfrak{n}(\mathfrak{g})$ se, e somente, se para todo $Y \in \mathfrak{g}$ tem-se:

$$
F(x \exp Y)=\left(e^{-\operatorname{ad}(Y)}\right) F(x)+\left(\frac{1-e^{-\operatorname{ad}(Y)}}{\operatorname{ad}(Y)}\right)(Y(F)+[Y, F(e)]),
$$

onde $Y(F)=d F_{e}(Y)$.

Demonstração: Defina primeiro a aplicação

$$
B: \mathfrak{g} \longrightarrow \mathfrak{g}, \quad B(Y)=Y(F)+[Y, F(e)]
$$

Considere $H \in C^{\infty}(G, \mathfrak{g})$ definido como em (4.10) que corresponde por sua vez ao campo vetorial $\widetilde{H}=[\widetilde{Y}, \widetilde{F}]$. Daí pode-se ver que para todo $Y \in \mathfrak{g}$

$$
B(Y)=H(e)
$$

Por outro lado,

$$
\begin{aligned}
\widetilde{F} & \in \mathfrak{n}(\mathfrak{g}) \Longleftrightarrow[\tilde{Y}, \widetilde{F}] \in \mathfrak{g}, \quad \forall Y \in \mathfrak{g} \\
& \Longleftrightarrow H(x)=\widetilde{Y}_{x}(F)+[Y, F(x)]=B(Y), \quad \forall x \in G \\
& \Longleftrightarrow \widetilde{Y}_{x}(F)=B(Y)-[Y, F(x)], \quad \forall x \in G \\
& \Longleftrightarrow\left(\frac{d}{d t}\right)_{t=0} F(x \exp t Y)=B(Y)-[Y, F(x)], \quad \forall x \in G \\
& \Longleftrightarrow\left(\frac{d}{d t}\right)_{t=0} F(x \exp t Y)=B(Y)-[Y, F(x \exp t Y)], \quad \forall x \in G .
\end{aligned}
$$

Agora, seja $f(t)=F(x \exp t Y)$. Então tomando a derivada desta função tem-se para todo $t \in \mathbb{R}$ e $x \in G$ que:

$$
\begin{aligned}
f^{\prime}(t) & =\left(\frac{d}{d t}\right)_{t=0} F(x \exp t Y)=B(Y)-[Y, F(x \exp t Y)], \quad \forall x, t \\
& =B(Y)-\operatorname{ad}(Y) f(t), \quad \forall x, t .
\end{aligned}
$$


Como $B(Y)$ não depende de $t$, segue pelo fato de ad $Y: \mathfrak{g} \rightarrow \mathfrak{g}$ é um homomorfismo de álgebras de Lie que

$$
\begin{aligned}
f^{\prime \prime}(t) & =-\operatorname{ad}(Y) f^{\prime}(t) \\
& =-\operatorname{ad}(Y)(B(Y)-\operatorname{ad}(Y) f(t)) \\
& =(-\operatorname{ad}(Y))^{2} f(t)+(-\operatorname{ad}(Y)) B(Y) .
\end{aligned}
$$

Para todo $n \in \mathbb{N}$ obtem-se então

$$
f^{(n)}(t)=(-\operatorname{ad}(Y))^{n} f(t)+(-\operatorname{ad}(Y))^{n-1} B(Y) .
$$

É claro que esta última equação é vpalida em particular para $n=1$. suponha que também seja verdadeira para $n$. Então,

$$
\begin{aligned}
f^{(n+1)}(t) & =\left(\frac{d}{d t}\right)_{t=0}\left\{(-\operatorname{ad}(Y))^{n} f(t)+(-\operatorname{ad}(Y))^{n-1} B(Y)\right\} \\
& =\left(\frac{d}{d t}\right)_{t=0}(-\operatorname{ad}(Y))^{n} f(t)+\left(\frac{d}{d t}\right)_{t=0}(-\operatorname{ad}(Y))^{n-1} B(Y) \\
& =(-\operatorname{ad}(Y))^{n+1} f(t)+(-\operatorname{ad}(Y))^{n} B(Y) .
\end{aligned}
$$

Pela indução vê-se que a equação em (4.13) é verdadeira. Analiticidade da função $f(t)$ é uma consequência imediata do fato que todo grupo de Lie é analítico. Daí usando a expansão em série de Taylor dessa função obtemos:

$$
f(t)=\sum_{n=0}^{\infty} \frac{t^{n}}{n !} f^{(n)}(0) .
$$

É fácil ver que $f^{(n)}(0)=(-\operatorname{ad}(Y))^{n} F(x)+(-\operatorname{ad}(Y))^{n-1} B(Y)$ para todo $n \geq 1$ e que $f(0)=f^{0}(0)=F(x)$. Então temos

$$
\begin{aligned}
f(t) & =\sum_{n=0}^{\infty} \frac{t^{n}}{n !}(-\operatorname{ad}(Y))^{n} F(x)+\sum_{n=1}^{\infty} \frac{t^{n}}{n !}(-\operatorname{ad}(Y))^{n-1} B(Y) \\
& =e^{-\operatorname{tad}(Y)} F(x)+\left(\frac{1-e^{-\operatorname{tad}(Y)}}{\operatorname{ad}(Y)}\right) B(Y) .
\end{aligned}
$$

Como $f(1)=F(x \exp Y)$, tem-se:

$$
\begin{aligned}
F(x \exp Y) & =e^{-\operatorname{ad}(Y)} F(x)+\left(\frac{1-e^{-\operatorname{ad}(Y)}}{\operatorname{ad}(Y)}\right) B(Y) \\
& =e^{-\operatorname{ad}(Y)} F(x)+\left(\frac{1-e^{-\operatorname{ad}(Y)}}{\operatorname{ad}(Y)}\right)(Y(F)+[Y, F(e)])
\end{aligned}
$$

o que completa a demonstração. 
Em particular, um elemento qualquer $\widetilde{F} \in \mathfrak{n}(\mathfrak{g})$ do normalizador pode ser caracterizado pelo valor da função correspondente $F$ na identidade $e \in G$ e sua diferencial $d F_{e}$.

Corolário 4.4.4. (Corolário 2.6, [3]) Todo elemento $\widetilde{F} \in \mathfrak{n}(\mathfrak{g})$ pode ser caracterizado pelo $\operatorname{par}\left(F(e), d F_{e}\right)$.

Demonstração: Let $U=\{g \in G: g=\exp Y$, por algum $Y \in \mathfrak{g}\}$. Como $G$ é conexo, tal vizinhança de $G$ que contém a identidade $e \in G$ gera o grupo todo, isto é, $\bigcup_{n \geq 1} U^{n}=G$. Pondo $x=e$ na fórmula (4.12) obtemos

$$
F(\exp Y)=e^{-\operatorname{ad}(Y)} F(e)+\left(\frac{1-e^{-\operatorname{ad}(Y)}}{\operatorname{ad}(Y)}\right)\left(d F_{e}(Y)+[Y, F(e)]\right)
$$

É claro que exp $Y \in U$ e a função $F$ é determinada pelo par $\left(F(e), d F_{e}\right)$ em $U$. Suponha que $F$ seja determinada pelo mesmo par, porém desta vez em $U^{n}$, e tome $x \in U^{n}$. Daí,

$$
x \exp Y \in U^{n} \times U=U^{n+1}
$$

e pelo Teorema 4.4 .3

$$
F(x \exp Y)=e^{-\operatorname{ad}(Y)} F(x)+\left(\frac{1-e^{-\operatorname{ad}(Y)}}{\operatorname{ad}(Y)}\right)\left(d F_{e}(Y)+[Y, F(e)]\right) .
$$

Portanto, $F(x)$ é de fato determinada pelo par $\left(F(e), d F_{e}\right)$ por um elemento $x$ em $U^{n}$ e logo $F(x \exp Y)$ também é determinada pelo par $\left(F(e), d F_{e}\right)$ em $U^{n+1}$.

Denote por $\operatorname{Dif}(G)$ o grupo de todos os difeomorfismos de $G$. Seja

$$
\alpha: G \otimes \operatorname{Aut}(G) \longrightarrow \operatorname{Dif}(G)
$$

a aplicação definida por $\alpha(x, \psi)=R_{x^{-1}} \cdot \psi$ e seja $\operatorname{Exp}: \mathfrak{n}(\mathfrak{g}) \rightarrow \operatorname{Dif}(G)$ a aplicação definida de tal modo que o diagrama a seguir comuta :

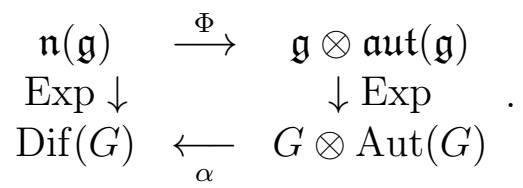

No Teorema abaixo caracteriza-se o grupo a 1-parâmetro $\tau_{t}$ de difeomorfismos de $G$ associado a $X \in \mathfrak{n}(\mathfrak{g})$. Segue, em particular, que qualquer elemento de $\mathfrak{n}(\mathfrak{g})$ possui um fluxo analítico global em $G$ associado a ele. Mais adiante, obteremos explicitamente em Teorema 4.4.6 as curvas integrais de $\widetilde{F} \in \mathfrak{n}(\mathfrak{g})$. 
Teorema 4.4.5. (i) A aplicação $\alpha: G \otimes \operatorname{Aut}(G) \rightarrow \operatorname{Dif}(G)$ é um homomorfismo injetor de grupos

(ii) Dado $X \in \mathfrak{n}(\mathfrak{g})$ o grupo a 1-parâmetro $\varphi_{t}$ de difeomorfismos de $G$ associado a $X$ é dado por

$$
\tau_{t}=\operatorname{Exp}(-t X)
$$

Em particular, qualquer elemento de $\mathfrak{n}(\mathfrak{g})$ possui um fluxo global analítico em $G$.

Demonstração: Provamos (i): Pelo produto semi-direto de grupos de Lie temos que para todo $x, y \in G$ e $\varphi, \psi \in \operatorname{Aut}(G)$

$$
\alpha((x, \varphi)(y, \psi))=\alpha(x \varphi(y), \varphi \circ \psi)=R_{(x \varphi(y))^{-1}}(\varphi \circ \psi)
$$

onde $R_{(x \varphi(y))^{-1}}=R_{x^{-1}} R_{\varphi(y)^{-1}}=R_{x^{-1}}\left(\varphi \circ R_{y^{-1}} \circ \varphi^{-1}\right)$. Obtemos, portanto,

$$
\alpha((x, \varphi)(y, \psi))=R_{x^{-1}}\left(\varphi \circ R_{y^{-1}} \circ \varphi^{-1}\right) \circ(\varphi \circ \psi)=\alpha(x, \varphi) \alpha(x, \psi)
$$

o que prova que $\alpha$ é, de fato, um homomorfismo de grupos. Segue então que $\alpha$ é injetora uma vez que $\alpha(x, \varphi)=I$ implica $e=R_{x^{-1}} \varphi(e)=x^{-1}$ e consequentemente $I=\alpha(e, \varphi)=\varphi$.

(ii) Como $\operatorname{Exp}(-t X)$ é um grupo a 1-parâmetro de difeomorfismos de $G$, basta computar o campo vetorial associado $\widetilde{H} \in X(G)$. Seja $g=\exp Y$ por algum $Y \in \mathfrak{g}$ e considere $g(t)=\operatorname{Exp}(-t X)(g)$. Sejam $\Phi(X)=(Z, D)$ e $\operatorname{Exp}(-t(Z, D))=\alpha(x(t), \varphi(t))$. Então

$$
g(t)=\operatorname{Exp}(-t(Z, D))(g)=\left(R_{x(t)^{-1}} \varphi(t)\right)(g)=(\varphi(t)(g)) x(t)^{-1} .
$$

Seja agora $\mu: G \times \operatorname{Aut}(G) \rightarrow G$ a aplicação definida por $\mu(x, \varphi)=\varphi(x)$. Então

$$
\begin{aligned}
\left(\frac{d}{d t}\right)_{t=0} \varphi(t)(g) & =d \mu_{(g, I)}(0, \dot{\varphi}(0))=d \mu_{(g, I)}(0,-D) \\
& =\left(\frac{d}{d t}\right)_{t=0} \mu(g, \operatorname{Exp}(-t D))=\left(\frac{d}{d t}\right)_{t=0}(\operatorname{Exp}(-t D))(\exp Y) \\
& =\left(\frac{d}{d t}\right)_{t=0} \exp \left(e^{-t D}(Y)\right)=d \exp _{Y}(-D(Y)) .
\end{aligned}
$$

Usando a equação (4.14) junto com o Teorema 1.7 de [24] tem-se:

$$
\dot{g}(0)=\left(\frac{d}{d t}\right)_{t=0} \varphi(t)(g)+\left(d L_{g}\right)_{e}\left(\frac{1-e^{-\mathrm{ad} Y}}{\mathrm{ad} Y}\right)\left(-D(Y)+\left(d L_{g}\right)_{e}(Z)\right) .
$$

Daí a função analítica $H \in C^{\infty}(G, \mathfrak{g})$ que corresponde ao campo vetorial $\widetilde{H}$ satisfaz:

$$
H(\exp Y)=\left(\frac{1-e^{-\operatorname{ad} Y}}{\operatorname{ad} Y}\right)(D(Y)+Z), \quad Y \in \mathfrak{g} .
$$


Pelo Teorema 4.4.3 e seu Corolário 4.4 .4 a função analítica $F \in C^{\infty}(G, \mathfrak{g})$ correspondente a $\widetilde{F}$ também satisfaz

$$
F(\exp Y)=\left(\frac{1-e^{-\operatorname{ad} Y}}{\operatorname{ad} Y}\right)(D(Y)+Z), \quad Y \in \mathfrak{g} .
$$

Segue portanto que $F(x)=H(x)$ para todo $x \in G$ e conclusa-se a esta altura a demonstração.

Dado $\widetilde{F} \in \mathfrak{n}(\mathfrak{g})$, seja $\Phi(\widetilde{F})=(E, \phi)$ onde $E=\sum_{j=1}^{m} u_{j} Y^{j} \in \mathfrak{g}$ e $\phi \in \mathfrak{D e r}(\mathfrak{g})$. Usando a fórmula de Campbell-Baker-Hausdorff pode-se obter uma fórmula explicíta de curvas integrais de $\widetilde{F}$ como:

Teorema 4.4.6. (Teorema 2.9, [3]) A curva integral $x(t)$ de $\widetilde{F} \in \mathfrak{n}(\mathfrak{g})$ a partir de $x \in G$ é dada numa vizinhança de $t=0$ por

$$
x(t)=\left(\operatorname{Exp}(-t \phi)(x) \exp \left(\sum_{n \geq 1}(-1)^{n+1} t^{n} d_{n}(E, \phi)\right)\right.
$$

onde $d_{n}: \mathfrak{g} \otimes \mathfrak{D} \mathfrak{e r}(\mathfrak{g}) \rightarrow \mathfrak{g}$ é uma aplicação polinomial homogênea de grau $n$ para todo $n \geq 1$.

Em particular, se $x=\exp X$ então

$$
x(t)=\exp \left(e^{-t \phi}(X)\right) \exp \left(\sum_{n \geq 1}(-1)^{n+1} t^{n} d_{n}(E, \phi)\right) .
$$

Demonstração: Veja a demonstração do Teorema 2.9 em [3].

Alguns dos primeiros termos de $d_{n}$ na fórmula acima são obtidos através de fórmula recursiva como

$$
\begin{aligned}
d_{1}(E, \phi) & =E \\
d_{2}(E, \phi) & =\frac{1}{2} \phi(E) \\
d_{3}(E, \phi) & =\frac{1}{12}[E, \phi(E)]+\frac{1}{6} \phi^{2}(E) \\
d_{4}(E, \phi) & =\frac{1}{24}\left[E, \phi^{2}(E)\right]+\frac{1}{24} \phi^{3}(E)
\end{aligned}
$$

e que a série $\sum_{n=1}^{\infty} d_{n}(E, \phi)$ é absolutamente convergente (e, portanto, convergente) numa vizinhança de $(0,0) \in \mathfrak{g} \otimes \mathfrak{D} \mathfrak{e r}(\mathfrak{g})$.

Observação 4.4.7. Em Proposição 1 de [9] cita-se um simples resultado de Cardetti [6], ou seja, dado um controle $u$, denote por $e(t)$ a solução de sistema de controle linear 
(4.2) a partir de identidade $e \in G$. Para condição inicial $g \in G$ a trajetória $g(t)$ é dada por

$$
g(t)=\varphi_{t}(g) e(t)
$$

onde $\varphi_{t}(g)$ é o fluxo induzido por campo drift $X$.

Ao nosso ver, tal Proposição não passa de uma re-formulação da fórmula dada em (4.15) por Ayala e Tirao. De fato,

$$
\zeta(t)=\zeta(t, u)=\sum_{n \geq 1}(-1)^{n+1} t^{n} d_{n}(E, \phi)
$$

é uma curva diferenciável no nível de álgebra de Lie passando por zero e, portanto, a sua imagem exponencial exp $\zeta(t)$ é a solução do sistema (4.2) começando na identidade do grupo correspondente, isto é, $e(t)$ de acordo com a notação mencionada acima. Por outro lado, $\operatorname{Exp}(-t \phi)(x)$ é nada mais nada menos que é o fluxo do campo linear $X$, ou seja, $\varphi_{t}(x)=\operatorname{Exp}(-t \phi)(x)$.

A tarefa de encontrar curvas integrais é, em geral, muito complicada. Então, a fórmula fornecida em (4.15) é extremamente difícil de calcular. Porém, pode ser calculada em alguns casos muito específicos. Por exemplo, sobre um grupo de Lie $G$ nilpotente e simplesmente conexo de tal modo que a referida fórmula torna-se finita cujos termos são relativamente fácil de computar. Escolher uma derivação nilpotente $D$ pode vir a ser útil nesse sentido. Note também que para grupos nilpotentes e simplesmente conexos a aplicação exponencial é um difeomorfismo global. Veja o exemplo a seguir onde temos feito alguns cálculos.

Exemplo 4.4.8. Seja $G$ o grupo de Heisenberg cuja álgebra de Lie é $\mathfrak{g}=\mathbb{R} Y^{1}+\mathbb{R} Y^{2}+$ $\mathbb{R} Y^{3}$ e considere sobre $G$ o seguinte sistema

$$
\Sigma: \dot{x}=X(x)+u Y^{2}(x), \quad u \in \mathbb{R}
$$

onde o automorfismo infinitesimal $X$ é definido por

$$
X_{t}\left(x_{1}, x_{2}, x_{3}\right)=\left(x_{1}+x_{2} t+\frac{1}{2} x_{2}^{2} t, x_{2}, t x_{2}+x_{3}\right) .
$$

De fato, usando a operação binária dada em $G$ pode ser checado facilmente que $X_{t}$ para todo $t \in \mathbb{R}$ é um automorfismo de $G$. Daí segue que o nosso sistema é dado como

$$
\begin{aligned}
& \dot{x}_{1}=x_{2}+\frac{1}{2} x_{2}^{2}+u x_{3} \\
& \dot{x}_{2}=u \\
& \dot{x}_{3}=x_{2} .
\end{aligned}
$$


Aplicando a fórmula (4.15) para

$$
E=u Y^{2} \quad \text { e } \quad \phi=\left(\begin{array}{ccc}
0 & 1 & 0 \\
0 & 0 & 0 \\
0 & 1 & 0
\end{array}\right) \in \mathfrak{D e r}(\mathfrak{g})
$$

(i.e. $\phi$ é a derivação que induz $X$ ) ve-se que $d_{n}$ se anula a partir de $n \geq 4$ já que $\phi$ é nilpotente de grau 2. Daí os termos não-nulos da série convergente são listados como

$$
d_{1}(E, \phi)=E, \quad d_{2}(E, \phi)=\frac{1}{2} u\left(Y^{1}+Y^{3}\right), \quad d_{3}(E, \phi)=-\frac{1}{12} u^{2} Y^{1} .
$$

Neste caso, obtemos a série como

$$
\zeta(t)=t d_{1}(E, \phi)-t^{2} d_{2}(E, \phi)+t^{3} d_{3}(E, \phi)
$$

e, portanto,

$$
\exp \zeta(t)=\exp \left(\left(-\frac{t^{3}}{12} u^{2}-\frac{t^{2}}{2} u\right) Y^{1}+u t Y^{2}-\frac{t^{2}}{2} u Y^{3}\right)
$$

Usando a regra de exponencial em $G$

$$
\exp (a X+b Y+c Z)=\left(a+\frac{1}{2} b c, b, c\right)
$$

a solução de $\Sigma$ a partir de um $x \in G$ é calculada como

$$
x(t)=\left(x_{1}+\left(x_{2}+\frac{1}{2} x_{2}^{2}+u x_{3}\right) t+\left(u x_{2}-\frac{u}{2}\right) t^{2}-\frac{t^{3}}{3} u^{2}, x_{2}+u t, t x_{2}+x_{3}-\frac{t^{2}}{2} u\right) .
$$

\subsection{Transitividade}

Seja $\Sigma$ um sistema de controle. Associado a $\Sigma$, temos um semigrupo $S_{\Sigma}$ gerado por $\Sigma$, ou semigrupo do sistema que é definido por

$$
S_{\Sigma}=\left\{X_{t_{1}}^{1} \circ X_{t_{2}}^{2} \circ \cdots \circ X_{t_{k}}^{k}: X^{i} \in \Sigma \text { e } t_{i} \in \mathbb{R}^{+}\right\}
$$

Notemos que $S_{\Sigma}$ é um semigrupo no sentido local. Pois, o produto é dado pela composta de difeomorfismos a menos de localização do domínio e as inversas dos elementos de $S_{\Sigma}$ não estão, em geral, em $S_{\Sigma}$ por exigência do tempo positivo.

De maneira semelhante, temos

$$
G_{\Sigma}=\left\{X_{t_{1}}^{1} \circ X_{t_{2}}^{2} \circ \cdots \circ X_{t_{k}}^{k}: X^{i} \in \Sigma \text { e } t_{i} \in \mathbb{R}\right\}
$$

o grupo (local) gerado pelos fluxos dos campos em $\Sigma$ sem restrição de que o tempo seja positivo. Portanto, este conjunto é fechado por composições e por elementos inversos, isto é, permite-se o retorno no tempo. De fato, $\left(X_{t}\right)^{-1}=X_{-t}$. 
A órbita pelo sistema em tempo positivo, ou simplesmente órbita positiva, a partir de $x \in M$ se define como a órbita de ação do semigrupo local $S_{\Sigma}$ sobre $x$, isto é,

$$
S_{\Sigma}(x)=\left\{\phi(x): \phi \in S_{\Sigma}\right\}
$$

O conjunto $S_{\Sigma}(x)$ também é conhecido como conjunto de acessibilidade ou conjunto acessível a partir de $x$. Temos sempre que $S_{\Sigma}(x) \subset G_{\Sigma}(x)$ embora ambas órbitas são completamente diferentes uma de outra. Pois, o fato de $S_{\Sigma}$ não ser um grupo faz com que as órbitas positivas $S_{\Sigma}(x)$ sejam objetos bem mais irregulares que as órbitas $G_{\Sigma}(x)$. Ao contrário das órbitas $G_{\Sigma}(x)$ que são subvariedades diferenciáveis de espaço estado (Veja Teorema de Órbita abaixo) o mesmo não vale em geral para $S_{\Sigma}(x)$. Assim, a propriedade dinâmica de irreversibilidade no tempo altera completamente a geometria da órbita positiva.

Uma outra diferença fundamental entre $S_{\Sigma}(x)$ e $G_{\Sigma}(x)$ está na relação de pertencer à mesma órbita:

$$
x \sim y \Longleftrightarrow y \in G_{\Sigma}(x) .
$$

É facil verificar que tal relação define uma relação de equivalência e mais ainda as suas classes de equivalência são nada mais nada menos que as folhas da folheação determinada por $\Sigma$. Já no caso do semigrupo ela deixa de ser simétrica devido ao proibição de retorno no tempo. Um caso bem particular em que $S_{\Sigma}(x)$ e $G_{\Sigma}(x)$ coincidem é quando o sistema $\Sigma$ é simétrico no sentido de que se $X \in \Sigma$ então $-X \in \Sigma$, isto é, $\Sigma=-\Sigma$.

Definição 4.5.1. Um sistema linear $\Sigma=(G, D)$ é dito controlável se $S_{\Sigma}(e)=G$ e transitivo se $G_{\Sigma}(e)=G$.

Exemplo 4.5.2. Seja $\Sigma=(G, \mathcal{D})$ onde $G=\mathbb{R}^{2}$ e a dinâmica $\mathcal{D}$ é dada pelos campos

$$
X^{1}=\left(\begin{array}{ll}
0 & 1 \\
0 & 0
\end{array}\right) \quad \text { e } \quad X^{2}=\left(\begin{array}{ll}
0 & 0 \\
1 & 0
\end{array}\right) .
$$

Os fluxos induzidos por $X^{1}$ e $X^{2}$ são

$$
X_{t}^{1}=\left(\begin{array}{cc}
1 & t \\
0 & 1
\end{array}\right) \quad \text { e } \quad X_{t}^{2}=\left(\begin{array}{cc}
1 & 0 \\
t & 1
\end{array}\right),
$$

respectivamente. Dada uma condição inicial $x_{0}=(a, b) \in G$, a órbita de $\Sigma$ é

$$
G_{\Sigma}\left(x_{0}\right)=\mathbb{R}^{2}
$$

enquanto que a órbita positiva é

$$
S_{\Sigma}\left(x_{0}\right)=\left\{(x, y) \in \mathbb{R}^{2}: x \geq a \text { e } y \geq b\right\} .
$$

Em outras palavras, o sistema é transitivo mas não é controlável. 
Exemplo 4.5.3. Seja $\Sigma=\left(\mathbb{R}^{2}, \mathcal{D}\right)$ onde a dinâmica é dada pelos campos de vetores

$$
X^{1}=\frac{\partial}{\partial x} \quad \text { e } \quad X^{2}=\left(\begin{array}{cc}
1 & 0 \\
0 & -1
\end{array}\right) .
$$

Dada uma condição inicial $x_{0}=(a, b) \in \mathbb{R}^{2}$, o fluxo $X_{t}^{1}: \mathbb{R}^{2} \rightarrow \mathbb{R}^{2}$ de $X^{1}$ é dado por $X_{t}^{1}(a, b)=(a+t, b), \forall t \in \mathbb{R}$. O fluxo de $X^{2}$ já foi encontrado no Exemplo 1.4.6 como $X_{s}^{2}(a, b)=\left(a e^{s}, b e^{-s}\right), \forall s \in \mathbb{R}$. Daí segue que as órbitas do sistema em consideração são como abaixo:

$$
\begin{aligned}
& G_{\Sigma}(a, b)=\{(x, y): y>0\} \text { se } b>0 \\
& G_{\Sigma}(a, b)=\{(x, 0): x \in \mathbb{R}\} \text { se } b=0 \\
& G_{\Sigma}(a, b)=\{(x, y): y<0\} \text { se } b<0
\end{aligned}
$$

enquanto que as as órbitas positivas são

$$
\begin{aligned}
& S_{\Sigma}(a, b)=\left\{(x, y): \frac{a b}{x} \leq y \leq b, x \geq a\right\} \text { se } b \geq 0 \\
& S_{\Sigma}(a, b)=\left\{(x, y): b \leq y \leq \frac{a b}{x}, x \geq a\right\} \text { se } b<0 .
\end{aligned}
$$

Em suma, o sistema não é transitivo nem controlável.

A seguir enunciamos um resultado clássico da Teoria sem demonstração que permite reduzir o espaço estado a sua órbita para toda condição inicial, mantendo toda a informação de dinâmica de sistema referente a condição inicial.

Teorema 4.5.4. (Teorema da órbita) Seja $\Sigma=(M, \mathcal{D})$ uma família de campos de vetores diferenciáveis sobre uma variedade diferenciável $M$, ou um sistema de controle em $M$. Então para cada $x \in M$ a órbita $G_{\Sigma}(x)$ possui uma estrutura de variedade diferencial tal que

(i) $\left\{G_{\Sigma}(x): x \in M\right\}$ é uma partição com singularidades de $M$

(ii) $\Sigma_{x}=\left(G_{\Sigma}(x), \mathcal{D}\right)$ é um sistema de controle transitivo sobre a órbita de $x$

(iii) A distribuição $\Delta$ definida por $\Delta=\left\{d \varphi\left(Y_{\varphi^{-1}(x)}\right): \varphi \in G_{\Sigma}\right\}$ é integrável e para todo $y \in G_{\Sigma}$ tem-se $\Delta(y)=T_{y} G_{\Sigma}(x)$, isto é, as variedades integrais da distribuição $\Delta$ são precisamente as órbitas de $\Sigma$.

Demonstração: Veja Sussmann [26] e Stefan [25].

Sejam $X, Y \in X(M)$ então o colchete $[X, Y]$ em cada ponto $y \in G_{\Sigma}(x)$ é dado por

$$
[X, Y](y)=\left(\frac{d}{d t}\right)_{t=0} Y_{-t} \circ X_{-t} \circ Y_{t} \circ X_{t}(y) .
$$

Como $X_{t}, Y_{s} \in G_{\Sigma}$ segue que para todo $t, s \in \mathbb{R}$ a curva $Y_{-t} \circ X_{-t} \circ Y_{t} \circ X_{t}(y) \in G_{\Sigma}(x)$ e, por conseguinte, o espaço tangente $T_{y} G_{\Sigma}(x)$ contém a sua derivada em $t=0$, isto é, $[X, Y](y) \in T_{y} G_{\Sigma}(x)$. 
Definição 4.5.5. Seja $\Sigma$ um sistema de controle em $M$. Definimos a álgebra de Lie $L(\Sigma)$ do sistema $\Sigma$ como

$$
L(\Sigma)=\operatorname{Lie}\left(X, Y^{1}, \ldots, Y^{m}\right)=\operatorname{Lie}(\mathcal{D})
$$

Se $\Sigma=(M, \mathcal{D})$ um sistema de controle então

$$
L(\Sigma)(x) \subset \Delta(x), \quad x \in M
$$

Em particular, para sistemas analíticos (i.e. sistemas compostos de campos analíticos) tem-se $L(\Sigma)(x)=\Delta(x)$ para todo $x \in M$. Daí segue que as órbitas de $\Sigma$ são as variedades integrais da distribuição (analítica) induzida por $L(\Sigma)$.

Para sistemas de controle em geral a propriedade de contolabilidade então significa : Sob quais circunstâncias sobre $M, \mathcal{D}$ e $x \in M$ a órbita positiva coincide com a órbita, ou seja,

$$
S_{\Sigma}(x)=G_{\Sigma}(x) .
$$

Em outras palavras, o Teorema da órbita permite considerarmos sistemas de controle transitivos sobre suas órbitas.

Relembramos que existe uma correspondência entre subgrupos de Lie conexos de $G$ e subálgebras de Lie de $\mathfrak{g}$ e que sob tal correspondência subgrupos normais são associados a ideias. Mais ainda, se $H$ é um subgrupo conexo de $G$ então cada $h \in H$ é um produto finito de elementos da forma $\exp (t Y)$ onde $Y$ pertence a álgebra de Lie $\mathcal{H}$ de $H$. Veja Jurdjevic-Sussmann [12].

Seja $\mathcal{H}$ a subálgebra de Lie de $\mathfrak{g}$ gerada pelos vetores do controle de $\Sigma$, a saber,

$$
\mathcal{H}=\operatorname{Lie}\left\{Y^{1}, \ldots, Y^{m}\right\}, \quad Y^{j} \in \mathfrak{g}
$$

Lema 4.5.6. Seja $G$ um grupo de Lie conexo com álgebra de Lie $\mathfrak{g}$. Se $X$ é um automorfismo infinitesimal então $\mathfrak{g}$ é uma subálgebra $\mathrm{ad}^{i}(X)$-invariante do normalizador para todo $i \geq 0$.

Demonstração: Dado $Y \in \mathfrak{g}$, provamos primeiro que $\operatorname{ad}(X)(Y) \in \mathfrak{g}$.

$$
[X, Y](e)=-Y(e) X=-\left(\frac{d}{d t}\right)_{t=0} X(\exp t Y)
$$

Sabemos que

$$
[X, Y](x)=\left(\frac{d}{d t}\right)_{t=0} d\left(X_{-t}\right)_{X_{t}(x)} Y\left(X_{t}(x)\right),
$$

onde,

$$
d\left(X_{-t}\right)_{X_{t}(x)} Y\left(X_{t}(x)\right)=\left(\frac{d}{d s}\right)_{s=0}\left(X_{-t} \circ \gamma\right)(s)
$$


sendo $\gamma$ uma curva tal que $\gamma(0)=X_{t}(x)$ e $\dot{\gamma}(0)=Y\left(X_{t}(x)\right)$. Então $\gamma(s)=X_{t}(x) \exp s Y$. Assim

$$
d\left(X_{-t}\right)_{X_{t}(x)} Y\left(X_{t}(x)\right)=\left(\frac{d}{d s}\right)_{s=0} X_{-t}\left(X_{t}(x)(\exp s Y)\right)
$$

e como $X_{t} \in \operatorname{Aut}(G)$, então $X_{-t}\left(X_{t}(x) \exp s Y\right)=x X_{-t}(\exp s Y)=L_{x}\left(X_{-t}(\exp s Y)\right)$, $\log \mathrm{O}$

$$
\begin{aligned}
{[X, Y](x) } & =\left(\frac{d}{d t}\right)_{t=0}\left(\frac{d}{d s}\right)_{s=0} L_{x}\left(X_{-t}(\exp s Y)\right) \\
& =-\left(\frac{d}{d s}\right)_{s=0}\left(\frac{d}{d t}\right)_{t=0} L_{x}\left(X_{t}(\exp s Y)\right)
\end{aligned}
$$

Aplicando a regra da cadeia, obtemos

$$
\begin{aligned}
& {[X, Y](x)=-\left.\left(\frac{d}{d s}\right)_{s=0}\left(d L_{x}\right)_{X_{t}(\exp s Y)}\right|_{t=0}\left(\frac{d}{d t}\right)_{t=0} X_{t}(\exp s Y)} \\
& =-\left(\frac{d}{d s}\right)_{s=0} d\left(L_{x}\right) \exp s Y X(\exp s Y) \\
& =\left.\left(d L_{x}\right) \exp s Y\right|_{s=0}\left(-\left(\frac{d}{d s}\right)_{s=0} X(\exp s Y)\right. \\
& =\left(d L_{x}\right)_{e}\left(-\left(\frac{d}{d s}\right)_{s=0} X(\exp s Y)\right.
\end{aligned}
$$

Por (4.16) temos que

$$
[X, Y](x)=\left(d L_{x}\right)_{e}\left(-Y_{e} X\right)=\left(d L_{x}\right)_{e}([X, Y](e))
$$

Portanto, $\mathfrak{g}$ é ad $(X)$-invariante.

Para $i=2$ devemos mostrar que para todo $Y \in \mathfrak{g}$,

$$
\operatorname{ad}^{2}(X)(Y)=[X,[X, Y]] \in \mathfrak{g}
$$

Mas $[X, Y] \in \mathfrak{g}$ pelo que foi mostrado no caso $i=1$, logo é claro que $[X,[X, Y]] \in \mathfrak{g}$, $\operatorname{assim} \mathfrak{g}$ é $\operatorname{ad}^{2}(x)$-invariante. O mesmo argumento vale para $i>2$.

Denotamos por $\langle X \mid \mathcal{H}\rangle$ a menor subalgebra $\operatorname{ad}(X)$-invariante de $\mathfrak{g}$ que contém $\mathcal{H}$, isto é,

$$
\langle X \mid \mathcal{H}\rangle=\operatorname{Lie}\left\{Y^{j}, \operatorname{ad}^{i}(X)\left(Y^{j}\right) \mid 1 \leq j \leq m, i \geq 0\right\},
$$

e por $H$ e $\langle X \mid H\rangle$ os subgrupos de Lie conexos de $G$ cujas álgebras de Lie são $\mathcal{H}$ e $\langle X \mid \mathcal{H}\rangle$, respectivamente.

Podemos escrever a álgebra de Lie $L(\Sigma)$ de $\Sigma$ como produto semi-direto de álgebras de Lie. 
Teorema 4.5.7. (Teorema 3.4, [3]) Seja $\Sigma=(G, \mathcal{D})$ um sistema linear tal que $X$ é um automorfismo infinitesimal. Então

$$
L(\Sigma) \cong\langle X \mid \mathcal{H}\rangle \otimes L(T)
$$

onde $T=\left\{X_{t} \mid t \in \mathbb{R}\right\}$.

Demonstração: Como $X_{t} \in \operatorname{Aut}(\langle X \mid H\rangle)$ podemos considerar a representação

$$
\tau: T \longrightarrow \operatorname{Aut}(\langle X \mid H\rangle)
$$

para construir produto semi-direto de $\langle X \mid H\rangle$ e $T$ com respecto a $\tau$. Denotamos tal grupo de Lie por

$$
S=\langle X \mid H\rangle \otimes T
$$

Segue então que $\rho=d \tau$ é uma representação de álgebra de Lie $L(T)$ de $T$ em $\langle X \mid \mathcal{H}\rangle$ tal que

$$
\rho(L(T))=\mathfrak{D} \mathfrak{e r}(\langle X \mid \mathcal{H}\rangle)
$$

Sobre o produto $\langle X \mid \mathcal{H}\rangle \otimes L(T)$ o colchete de Lie

$$
\left[\left(Y_{1}, W_{1}\right),\left(Y_{2}, W_{2}\right)\right]=\left(\left[Y_{1}, Y_{2}\right]+\rho\left(W_{1}\right)\left(Y_{2}\right)-\rho\left(W_{2}\right)\left(Y_{1}\right),\left[W_{1}, W_{2}\right]\right)
$$

é bem definido e transforma este produto em uma álgebra de Lie chamada produto semi-direto de $\langle X \mid \mathcal{H}\rangle$ com $L(T)$ com respecto a $\rho$. Denotamos esta álgebra de Lie por

$$
\mathfrak{s}=\langle X \mid \mathcal{H}\rangle \otimes L(T)
$$

Como $L(T)=\operatorname{Span}\{X\}$ e $[X, X]=0$ segue que $L(T)$ é uma álgebra abeliana. Por outro lado, dados $Y_{1}, Y_{2} \in\langle X \mid \mathcal{H}\rangle$ e $W_{1}, W_{2} \in L(T)$ temos por (4.17) que

$$
\begin{gathered}
\left.\left[\left(Y_{1}, W_{1}\right),\left(Y_{2}, W_{2}\right)\right]=\left(\left[Y_{1}, Y_{2}\right]+\left[W_{1}, Y_{2}\right]-\left[W_{2}, Y_{1}\right], 0\right]\right) \in\langle X \mid \mathcal{H}\rangle \otimes\{0\} \cong\langle X \mid \mathcal{H}\rangle, \text { (4.18) } \\
\text { uma vez que } \rho(W)(Y)=[W, Y] \text { para todo } W \in L(T) \text { e } Y \in\langle X \mid \mathcal{H}\rangle \text {. Portanto temos } \\
{[\mathfrak{s}, \mathfrak{s}] \subset\langle X \mid \mathcal{H}\rangle .}
\end{gathered}
$$

Tomamos a álgebra de Lie $L(\Sigma)$ do sistema $\Sigma$

$$
L(\Sigma)=\operatorname{Lie}\left\{X, Y^{1}, \ldots, Y^{m}\right\}
$$

e consideramos a aplicação

$$
\theta: L(\Sigma) \longrightarrow\langle X \mid \mathcal{H}\rangle \otimes L(T), \quad X+Y^{j} \longrightarrow\left(Y^{j}, X\right) .
$$


Mostramos que esta aplicação estabelece um isomorfismo de álgebras de Lie. De fato, devido a propriedade de bilinearidade de colchete em $X(G)$ bastar escolher dois elementos genéricos do tipo $X+Y^{i}$ e $X+Y^{j}$ e verificar que

$$
\theta\left[X+Y^{i}, X+Y^{j}\right]=\left[\theta\left(X+Y^{i}\right), \theta\left(X+Y^{j}\right)\right] .
$$

Com efeito,

$\theta\left[X+Y^{i}, X+Y^{j}\right]=\theta\left(\left[Y^{i}, Y^{j}\right]+\left[Y^{i}, X\right]+\left[X, Y^{j}\right]\right)=\left(\left[Y^{i}, Y^{j}\right]+\left[Y^{i}, X\right]+\left[X, Y^{j}\right], 0\right)$

e temos por (4.18) e pela definição da aplicação $\theta$ que

$$
\begin{aligned}
{\left[\theta\left(X+Y^{i}\right), \theta\left(X+Y^{j}\right)\right] } & =\left[\left(Y^{i}, X\right),\left(Y^{j}, X\right)\right] \\
& =\left(\left[Y^{i}, Y^{j}\right]+\left[X, Y^{j}\right]-\left[X, Y^{i}\right], 0\right) \\
& =\left(\left[Y^{i}, Y^{j}\right]+\left[X, Y^{j}\right]+\left[Y^{i}, X\right], 0\right) .
\end{aligned}
$$

Assim

$$
L(\Sigma) \cong\langle X \mid \mathcal{H}\rangle \otimes L(T)
$$

terminando a demonstração.

Sabe-se pela Proposição 2.3.11 que a álgebra de Lie de produto semi-direto de grupos de Lie é isomorfo a produto de suas respectivas álgebras de Lie. Em outras palavras, podemos enunciar o seguinte

Corolário 4.5.8. Seja $\Sigma=(G, \mathcal{D})$ um sistema de controle linear. Então

$$
L(\Sigma) \cong L(\langle X \mid H\rangle \otimes T) .
$$

Definição 4.5.9. (Condição de posto de álgebra de Lie) Diz-se que um sistema linear $\Sigma=(G, \mathcal{D})$ satisfaz a condição de posto de álgebra de Lie se

$$
\operatorname{dim}(\langle X \mid \mathcal{H}\rangle)=\operatorname{dim}(G) .
$$

Teorema 4.5.10. (Teorema 3.3, [3]) A órbita de $\Sigma$ a partir de identidade e $\in G$ é $\langle X \mid H\rangle$. Mais ainda, $\Sigma$ é transitivo se, e somente, se satisfaz a condição de posto de álgebra de Lie.

Demonstração: Como $L(T)$ é uma álgebra de Lie abeliana

$$
\langle X \mid \mathcal{H}\rangle \cong L(\Sigma)(e)
$$

Como foi dito antes, as órbitas de sistemas de controle analíticos são as variedades integrais da distribuição gerada por $L(\Sigma)$. Como consequência disso temos

$$
G_{\Sigma}(e)=\langle X \mid H\rangle
$$


e segue pela hipótese de $\Sigma$ ser transitivo que

$$
G_{\Sigma}(e)=\langle X \mid H\rangle=G .
$$

Daí podemos deduzir que $\langle X \mid \mathcal{H}\rangle=\mathfrak{g}$ o que por sua vez implica que

$$
\operatorname{dim}(\langle X \mid \mathcal{H}\rangle)=\operatorname{dim} \mathfrak{g}=\operatorname{dim}(G) .
$$

Reciprocamente, se $\operatorname{dim}(\langle X \mid \mathcal{H}\rangle)=\operatorname{dim}(G)$ então $\langle X \mid \mathcal{H}\rangle=\mathfrak{g}$ e, por conseguinte, $G_{\Sigma}(e)=\langle X \mid H\rangle=G$.

O seguinte resultado clássico de L. Markus é um caso particular do Teorema acima.

Teorema 4.5.11. (L. Markus, [15]) Seja $\Sigma$ um sistema de controle linear sobre o subgrupo de Lie matricial $G \subset \operatorname{Gl}(n, \mathbb{R})$ de dimensão $n^{2}$ com a dinâmica dada pela familia de equações diferenciais em $G$ da forma

$$
\dot{P}=X P-P X+\sum_{j=1}^{m} u_{j} Y^{j} P
$$

onde $X^{*}(P)=X P-P X$. Se $\Sigma$ é controlável em $G$, então $\Sigma$ satisfaz a condição de posto de álgebra de Lie.

O resultado do Teorema 4.5.10 acima mostra pra gente que a condição de posto de álgebra de Lie é suficiente para garantir transitividade do sistema em considereação mas não é necessária para controlabilidade de $\Sigma$ uma vez que $S_{\Sigma}(e) \subset G_{\Sigma}(e)$. Ou seja, tal condição não caracteriza propriedade de controlabilidade de sistemas lineares em grupos de Lie ao contrário de sistemas lineares em espaços Euclideanos.

Veja o seguinte exemplo sobre o grupo de Heisenberg nilpotente e simplesmente conexo de dimensão 3 onde sistema é transitivo mas não é controlável.

Exemplo 4.5.12. Seja $G$ o grupo nilpotente e simplesmente conexo de Heisenberg de dimesão 3. A álgebra de Lie de $G$ é $\mathfrak{g}=\mathbb{R} Y^{1}+\mathbb{R} Y^{2}+\mathbb{R} Y^{3}$ onde os geradores $Y^{1}, Y^{2}$ e $Y^{3}$ são operadores diferenciais de primeira ordem definidas por

$$
Y^{1}=\frac{\partial}{\partial x_{1}}, \quad Y^{2}=\frac{\partial}{\partial x_{2}}+x_{1} \frac{\partial}{\partial x_{1}}, \quad Y^{3}=\frac{\partial}{\partial x_{3}} .
$$

O único colchete de Lie que não se anula é $\left[Y^{1}, Y^{2}\right]=Y^{3}$.

Seja $\Sigma$ o seguinte sistema linear em $G$

$$
\dot{x}(t)=X(x(t))+u Y^{2}(x(t)), \quad u \in \mathbb{R},
$$

onde $X$ é o automorfismo infinitesimal induzida pela derivação

$$
D=\left(\begin{array}{ccc}
0 & 1 & 0 \\
1 & 0 & 0 \\
0 & 0 & 0
\end{array}\right) \in \mathfrak{D} \mathfrak{e r}(\mathfrak{g}) .
$$


O sistema $\Sigma$ em coordenadas é da forma

$$
\begin{aligned}
& \dot{x}_{1}(t)=x_{2}(t)+\frac{1}{2} x_{3}(t)+\frac{1}{2} x_{1}(t)-\frac{1}{4} x_{2}(t) x_{3}(t) \\
& \dot{x}_{2}(t)=x_{1}(t)-\frac{1}{2} x_{2}(t) x_{3}(t)+u \\
& \dot{x}_{3}(t)=0
\end{aligned}
$$

Observe que em [3] este sistema aparece como

$$
\begin{aligned}
& \dot{x}_{1}(t)=x_{2}(t) \\
& \dot{x}_{2}(t)=x_{1}(t)+u(t) \\
& \dot{x}_{3}(t)=0
\end{aligned}
$$

o que é, na verdade, a linearização de $\Sigma$ e não o próprio sistema.

Como $\mathcal{H}=\operatorname{Span}\left\{Y^{2}\right\}$ um simples cálculo mostra que $\left[X, Y^{2}\right]=Y^{1}$ e $\left[X, Y^{1}\right]=Y^{2}$. Daí obtemos

$$
\operatorname{ad}(X)(\mathcal{H})=\operatorname{Span}\left\{Y^{1}, Y^{2}\right\}
$$

e consequentemente a condição de posto de álgebra de Lie é satisfeita, isto é,

$$
\langle X \mid \mathcal{H}\rangle=\operatorname{Lie}\left\{Y^{1}, Y^{2}\right\}=\operatorname{Span}\left\{Y^{1}, Y^{2}, Y^{3}\right\}=\mathfrak{g} .
$$

Geometricamente falando, uma vez que as trajetórias partindo da origem atingem o plano horizontal- $x_{1} x_{2}$ elas ficam presas lá e, portanto, não há controlabilidade local embora o sistema é transitivo.

\subsection{Controlabilidade Local}

O problema de controlabilidade de sistemas não-lineares é em geral muito difícil razão pela qual consta na literatura resultados parciais para casos específicos. Recentemente, a questão de controlabilidade local tornou-se importante e chamou a atenção de diversos teoristas de controle. A seguir apresentaremos um resultado de controlabilidade local na identidade para a classe de sistemas lineares em grupos de Lie.

Definimos indutivamente $\operatorname{ad}(X)(\mathcal{H})$-sequência como:

$$
\begin{gathered}
\mathcal{H}_{0}=\mathcal{H} \\
\mathcal{H}_{1}=\mathcal{H}_{0}+\operatorname{ad}(X)(\mathcal{H}) \\
\mathcal{H}_{2}=\mathcal{H}_{1}+\operatorname{ad}^{2}(X)(\mathcal{H}) \\
\vdots \\
\mathcal{H}_{i}=\mathcal{H}_{i-1}+\operatorname{ad}^{i}(X)(\mathcal{H}),
\end{gathered}
$$

onde $\operatorname{ad}^{i}(X)(\mathcal{H})=\left\{\left[X, \operatorname{ad}^{i-1}(X)(Y)\right] \mid Y \in \mathcal{H}\right\}$. O Lema 4.5.6 permite mostrar o seguinte, 
Proposição 4.6.1. $\mathcal{H}_{i}$ é um subespaço vetorial de $\mathfrak{g}$ tal que $\mathcal{H}_{i} \subset \mathcal{H}_{i+1}$.

Demonstração: Seja $i=1$. Temos

$$
\mathcal{H}_{1}=\mathcal{H}+\operatorname{ad}(X)(\mathcal{H})
$$

Por definição $\mathcal{H}$ é um subespaço vetorial, pois é uma subálgebra de $\mathfrak{g}$ então basta mostrar que $\operatorname{ad}(X)(\mathcal{H})$ é um subespaço de $\mathfrak{g}$. Para isso, observe primeiro que como $\mathcal{H}$ é um subespaço de $\mathfrak{g}$, e $\mathfrak{g}$ é $\operatorname{ad}(X)$-invariante, então $\operatorname{ad}(X)(\mathcal{H}) \subset \mathfrak{g}$.

Sejam $\left[X, Z^{1}\right]$ e $\left[X, Z^{2}\right]$ elementos de $\operatorname{ad}(X)(\mathcal{H})$ e $\alpha, \beta \in \mathbb{R}$. Como o colchete é bilinear temos,

$$
\alpha\left[X, Z^{1}\right]+\beta\left[X, Z^{2}\right]=\left[X, \alpha Z^{1}+\beta Z^{2}\right]
$$

onde $\alpha Z^{1}+\beta Z^{2} \in \mathcal{H}$ pois $\mathcal{H}$ é um subespaço e, portanto, $\alpha\left[X, Z^{1}\right]+\beta\left[X, Z^{2}\right] \in$ $\operatorname{ad}(X)(\mathcal{H})$, isto é, $\operatorname{ad}(X)(\mathcal{H})$ e um subespaço de $\mathfrak{g}$, logo $\mathcal{H}_{1}$ é um subespaço de $\mathfrak{g}$.

Por indução, para $i>1$ suponha que $\mathcal{H}_{k}=\mathcal{H}_{k-1}+a d^{k}(X)(\mathcal{H})$ é um subespaço de $\mathfrak{g}$, queremos mostrar que vale a igualdade para $k+1$. Com efeito,

$$
\mathcal{H}_{k+1}=\mathcal{H}_{k}+a d^{k+1}(X)(\mathcal{H})
$$

e como $\mathcal{H}$ é um subespaço de $\mathfrak{g}$ enquanto $\mathfrak{g}$ é $a d^{k+1}(X)$-invariante segue então que $a d^{k+1}(X)(\mathcal{H}) \subset \mathfrak{g}$. Sejam $\left[X, a d^{k}(X)\left(Z^{1}\right)\right]$ e $\left[X, a d^{k}(X)\left(Z^{2}\right)\right]$ elementos de $a d^{k+1}(X)(\mathcal{H})$ e $\alpha, \beta \in \mathbb{R}$. Daí

$$
\alpha\left[X, a d^{k}(X)\left(Z^{1}\right)\right]+\beta\left[X, a d^{k}(X)\left(Z^{2}\right)\right]=\left[X, a d^{k}(X)\left(\alpha Z^{1}+\beta Z^{2}\right)\right]
$$

onde $\alpha Z^{1}+\beta Z^{2} \in \mathcal{H}$ e, por conseguinte, $\alpha\left[X, a d^{k}(X)\left(Z^{1}\right)\right]+\beta\left[X, a d^{k}(X)\left(Z^{2}\right)\right] \in$ $a d^{k+1}(X)(\mathcal{H})$. Portanto $\mathcal{H}_{k+1}$ é um subespaço de $\mathfrak{g}$. E por último, é facil ver que

$$
\mathcal{H} \subset \mathcal{H}_{1} \subset \cdots \subset \mathcal{H}_{i} \subset \cdots
$$

Como estamos considerando grupos de Lie com dimensao finita e $\mathcal{H} \neq 0$, então a Proposição 4.6.1 mostra que existe um menor inteiro $p \in \mathbb{N}, 0 \leq p<\operatorname{dim}(G)$ tal que a $\operatorname{ad}(X)$-sequência se estabiliza em $p$, ou seja,

$$
\mathcal{H}_{p}=\mathcal{H}_{p+q} \quad \text { para todo } q \in \mathbb{N} \text {. }
$$

Proposição 4.6.2. $\mathcal{H}_{p}$ é um subespaço ad $(X)$-invariante de $\mathfrak{g}$ que contém $\mathcal{H}$.

Demonstração: $\mathcal{H} \subset \mathcal{H}_{p}$ e $\mathcal{H}_{p}=\mathcal{H}_{p+q}, \forall q \geq 0$ 
Como sabemos, $\mathcal{H}_{i}$ é o subespaço gerado por $\mathcal{H}, \operatorname{ad}(X)(\mathcal{H}), \ldots, \operatorname{ad}^{i}(X)(\mathcal{H})$. Portanto, $Y^{1},\left[X, Y^{2}\right] \in \mathcal{H}_{i}$ mas $\left[Y^{1},\left[X, Y^{2}\right]\right]$ não necessariamente pertence a $\mathcal{H}_{i}$ mas certamente está em $\mathfrak{g}$. Como

$$
\mathcal{H}_{p}=\operatorname{Span}\left\{Y^{j}, \operatorname{ad}^{i}(X)\left(Y^{j}\right) \mid 1 \leq j \leq m \text { e } 0 \leq i \leq p\right\}
$$

devemos notar que o automorfismo infinitesimal $X$ não pertence a $\mathcal{H}_{p}$ e que pela Proposição 4.6.1 tem-se:

$$
\mathcal{H}_{p} \subset\langle X \mid \mathcal{H}\rangle \subset \mathfrak{g}
$$

Definição 4.6.3. (Controlabilidade Local) Um sistema linear $\Sigma$ em G é dito localmente controlável em $x_{0} \in G$ se $x_{0} \in \operatorname{int} S_{\Sigma}\left(x_{0}\right)$.

Um dos resultados mais conhecidos da teoria [17] garante que um sistema de controle não-linear é localmente controlável a partir de um ponto de equilíbrio sempre que a sua linearização for controlável. Pela Definição acima, controlabilidade local a partir de um ponto exige que tal ponto esteja no interior de sua órbita positiva ao contrário de controlabilidade para qual a órbita positiva é o espaço estado todo.

Todos esses comentários dão origem a seguinte condição de posto mais forte:

Definição 4.6.4. (Condição de posto) Dado um sistema de controle linear $\Sigma$ em $G$ diz-se que $\Sigma$ satisfaz a condição do posto se

$$
\operatorname{dim}\left(\mathcal{H}_{p}\right)=\operatorname{dim}(G)
$$

O seguinte resultado de controlabilidade local foi obtido em [3]:

Teorema 4.6.5. Seja $G$ um grupo de Lie conexo e $\Sigma=(G, \mathcal{D})$ um sistema transitivo tal que o campo drift $X$ é um automorfismo infinitesimal. Se $\Sigma$ satisfaz a condição do posto então $\Sigma$ é localmente controlável a partir de identidade $e \in G$.

Demonstração: Seja $x(t, u)$ a solução da equação diferencial

$$
\dot{x}(t)=X(x)+\sum_{j=1}^{m} u_{j} Y^{j}(x)
$$

parametrizada por $u \in \mathcal{U}_{m} \subset L_{\infty}\left([0, t], \mathbb{R}^{m}\right)$ passando pela identidade $e \in G$. Dado $\mathrm{u}$ $t>0$ considere a aplicação de ponto final

$$
E_{t}: \mathcal{U}_{m} \longrightarrow G
$$

definida por $E_{t}(u)=x(t, u)$. Esta aplicação é diferenciável [2] e a sua derivada $\left(d E_{t}\right)_{0}$ em $t=0$ é definida numa vizinhança $B$ de controle constante $u \equiv 0$ e é dada por

$$
\left(d E_{t}\right)_{0}(u(\cdot))=\int_{0}^{t} e^{(t-\tau) \operatorname{ad}(X)}\left(\sum_{j=1}^{m} u_{j}(\tau) Y_{e}^{j}(x)\right) d \tau .
$$


Suponha agora que exista um vetor $w$ no espaço cotangente de tal modo que

$$
\left\langle w,\left(d E_{t}\right)_{0}(u(\cdot))\right\rangle=0
$$

para todo $u(\cdot) \in B$. Em particular,

$$
\int_{0}^{t} \sum_{j=1}^{m}\left\langle w, e^{(t-\tau) \operatorname{ad}(X)} Y_{e}^{j}\right\rangle u_{j}(\tau) d \tau=0 .
$$

Como a equação (4.19) vale para toda função constante por pedaços $u:[0, t] \rightarrow \mathbb{R}^{m}$ podemos concluir que

$$
\left\langle w, e^{(t-\tau) \operatorname{ad}(X)} Y_{e}^{j}\right\rangle=0
$$

para todo $\tau \in[0, t]$. Tomando a derivada obtemos para cada $i \geq 0$ e $j=1, \ldots, m$ que

$$
\left\langle w, \operatorname{ad}^{i}(X) Y_{e}^{j}\right\rangle=0
$$

o que contradiz com a condição de posto. Portanto, a aplicação linear $\left(d E_{t}\right)_{0}$ é sobrejetora. Daí segue pelo Teorema de Função Implícita que existe uma vizinhança $U$ de $e$ em $G$ tal que $U \subset S_{\Sigma}(e)$ e, consequentemente, $\Sigma$ é localmente controlável em $e$. Pela transitividade de $\Sigma$ também segue que $d L_{x}\left(\mathcal{H}_{p}\right)$ gera o espaço tangente de $G$ em todo $x \in G$. Argumentos análogos implicam que $\Sigma$ é localmente controlável em $G$.

Corolário 4.6.6. Seja $G$ um grupo de Lie conexo e $\Sigma=(G, \mathcal{D})$ um sistema linear transitivo tal que $X$ é um automorfismo infinitesimal.

(i) Se G é abeliano então $\Sigma$ é controlável se, e somente, se $\Sigma$ satisfaz a condição de posto

(ii) Se $G$ é compacto e $\Sigma$ satisfaz a condição de posto então $\Sigma$ é controlável.

Demonstração: Pela hipótese, a álgebra de Lie $\mathfrak{g}$ de $G$ é abeliana e $X_{t} \in \operatorname{Aut}(G)$ para todo $t \in \mathbb{R}$. Em particular, a órbita positiva $S_{\Sigma}(e)$ é um semigrupo. Como $\mathcal{H}_{p}=\mathfrak{g}$ então segue pelos Teoremas 4.5.10 e 4.6.5 que $\Sigma$ é localmente controlável se, e somente, se $\Sigma$ satisfaz a condição de posto. Seja $U$ uma vizinhança de $e$ contida em $S_{\Sigma}(e)$. Como todo grupo de Lie conexo é gerado por uma vizinhança de identidade segue que

$$
\bigcup_{m} U^{m}=G
$$

e portanto provamos a primeira afirmação feita. Quanto ao segundo item, sabemos pelo Teorema 4.6.5 que existem vizinhanças $U_{1}, \ldots, U_{m}$ em $G$ tais que $G=U_{1} \cup \cdots \cup U_{m}$ e que $\Sigma$ é localmente controlável em cada $U_{j}, j=1, \ldots, m$. Em particular, $\Sigma$ é controlável.

Observação 4.6.7. Em [7] a seguinte versão fraca do resultado acima foi dada usando a teoria de semigrupos de Lie: sob condição de posto a órbita é uma vizinhança de identidade $e$. 
Considerações Finais

Em [3] foi conjecturado que órbita de sistemas lineares em grupos de Lie não são em geral semigrupos ao contrário de sistemas invariantes em grupos de Lie. Em (Proposição $7,[9]$ ) foi obtido um resultado mais forte dizendo que a órbita não pode ser um semigrupo a não ser o próprio grupo.

Um importante resultado de equivalência foi apresentado recentemente por P. Jouan em [10] onde o objetivo era o de caracterizar a classe de sistemas de controle afim

$$
\dot{x}(t)=f(x)+\sum_{j=1}^{m} u_{j} g_{j}(x), \quad x \in M,
$$

que são globalmente difeomorfos a um sistema linear em um grupo de Lie ou um espaço homogêneo. A resposta é que tais sistemas são compostos de campos completos que geram uma álgebra de Lie de dimensão finita.

Foi estabelecido também em (Teorema 4, [9]) um resultado de controlabilidade para sistemas lineares em grupos de Lie semi-simples. A saber, um sistema linear $\Sigma$ em um grupo de Lie $G$ semi-simples com $X$ interno (i.e, $X=\operatorname{ad}(Z)$ para algum $Z \in \mathfrak{g}$ ) é controlável se, e somente, se a subálgebra $\mathfrak{h}$ gerada por campos controlados $Y^{j}$ de $\Sigma$ coincide com a álgebra de Lie toda $\mathfrak{g}$. A condição usada nesse Teorema para garantir controlabilidade global é idéntica com o resultado inicialmente obtido por Ayala em [4] para sistemas invariantes em grupos de Lie nilpotentes e simplesmente conexos que posteriormente foi generalizado por Y. Sachkov em [21] para grupos de Lie completamente solúveis. 



\section{Referências Bibliográficas}

[1] A. A. Agrachev and Yu. L. Sachkov, Control Theory from the Geometric Viewpoint, Springer, 2004.

[2] A. A. Agrachev, R. V. Gamkrelidze and A. V. Sarychev, Local Invariants of Smooth Control Systems, Acta Applicandae Mathematicae 14 (1989), 191-237.

[3] V. Ayala and J. Tirao, Linear control systems on Lie groups and controllability. Proceedings of the American Mathematical Society, Series: Symposia in Pure Mathematics 64 (1999), 47-64.

[4] V. Ayala, Controllability of nilpotent systems. Geometry in Nonlinear Control and Differential Inclusions, Banach Center Publications, Warszawa, Poland, (1995).

[5] Colonius F. and W. Kliemann, The Dynamics of Control, Birkhäuser (2000).

[6] F. Cardetti, On Properties of Linear Control Systems on Lie Groups, Ph.D Thesis, Louisiana State University, 2002.

[7] F. Cardetti and D. Mittenhuber, Local Controllability for Linear Systems on Lie Groups, Journal of Dynamical and Control Systems 11 (2005), 353-373.

[8] E. A. Codington and N. Levinson, Theory of Ordinary Differential Equations, McGraw-Hill, 1955.

[9] P. Jouan, Controllability of Linear Systems on Lie groups, Journal of Dynamical and Control Systems 17, (2011) 591-616.

[10] P. Jouan, Equivalence of Control Systems with Linear Systems on Lie groups and Homogenous Spaces, ESAIM:Control Optimization and Calculus of Variation 16, (2010) 956-973. 
[11] V. Jurdjevic, Geometric Control Theory, Cambridge University Press (1997).

[12] V. Jurdjevic and H. Sussmann, Control Systems on Lie Groups, Differential Equations 12 (1972), 313-329.

[13] R. Kalman, Y. Ho and K. Narendra, Controllability of linear dynamical systems, Contrib. to Diff. Equations 1 (2) (1962), 189-213.

[14] J. Macki, Introduction to Optimal Control Theory, Springer (1995).

[15] L. Markus, Controllability of multi-trajectories on Lie group, Proceedings of Dynamical Systems and Turbulence, Lecture Notes in Mathematics 898, (1980) 250-265

[16] F. Manfio, Variedades Diferenciáveis, Notas de Aula, 2011.

[17] H. Nijmeijer and A. J. van der Schaft, Non linear dynamical control systems, Springer-Verlag (1990).

[18] R. Zegarra, El normalizador del álgebra de Lie de un grupo y una nueva clase de sistemas de control, Dissertação de Mestrado, Universidad Católica del Norte, Chile, 1996.

[19] E. O. Roxin, Control Theory and its Applications, Gordon and Breach Science Publichers, 1997.

[20] Y. Sachkov, Controllability of Invariant Systems on Lie Groups and Homogeneous Spaces. Progress in Science and Technology, Series on Contemporary Mathematics and Applications, Thematical Surveys, Vol. 59, 1998.

[21] Y. Sachkov, Controllability of affine right-invariant systems on solvable Lie groups. Discrete Mathematics and Theoretical Computer Science 1 (1997) 239-246.

[22] L. San Martin, Grupos de Lie, Notas de Aula, Imecc-Unicamp.

[23] L. San Martin, Álgebras de Lie, Editora Unicamp, 2a edição, 2011.

[24] S. Helgason, Differential Geometry and Symmetric Spaces, Academic Press, 1962.

[25] P. Stefan, Accessible sets, orbits, and foliations with singularities, J. London Math. Soc. (1974), 699-713.

[26] H. Sussmann, Orbits of families of vector fields and integrability of distributions, Trans. Amer. Math. Soc., 180 (1973), 171-188. 


\section{Índice Remissivo}

álgebra

de Heisenberg, 40

de Lie, 39

de produto direto de grupos de Lie,

40

de sistema, 61, 90

especial ortogonal $\mathfrak{s o}(3), 41$

linear $\mathfrak{g l}(n, \mathbb{R}), 39$

órbita, 88

positiva, 57, 88

teorema da, 89

aplicação exponencial, 50

atlas, 17

maximal, 17

automorfismo

de álgebras de Lie, 44

de grupos de Lie, 38

infinitesimal, 69

interno, 36

campo vetorial, 21

completo, 27

drift, 71

invariante, 46

linear, 68, 70

centralizador, 44, 72

centro, 44 colchete de Lie, 23

invariância de , 48

condição

de Kalman, 61

de posto, 97

de posto de álgebra de Lie, 93

conjunto

acessível, 32

de acessibilidade, 55

controlabilidade, 57

local, 97

matriz de , 61

controle, 30

bang-bang, 30

constante por pedaço, 30

curva integral, 25

maximal, 26

derivação, 22

de Lie, 42

interna, 42

derivada de Lie, 29

espaço

de derivações, 22

de derivações de Lie $\mathfrak{D} \mathfrak{e r}(\mathfrak{g}), 42$

estado, 30

tangente, 19 
fórmula

de Campbell-Baker-Hausdorf, 50

de variação de parâmetro, 55

fibrado tangente, 20

fluxo

local, 25

maximal, 27

grupo

a 1-parâmetro, 27

de automorfismos de um grupo de Lie $\operatorname{Aut}(G), 38$

de automorfismos de uma álgebra de

Lie $\operatorname{Aut}(\mathfrak{g}), 44$

de Heisenberg, 36

de Lie, 35

linear $\operatorname{Gl}(n, \mathbb{R}), 36$

linear especial $\mathrm{Sl}(n, \mathbb{R}), 38$

local a 1-parâmetro, 27

ortogonal $\mathrm{O}(n), 37$

ortogonal especial $\mathrm{SO}(n), 37$

simplético real $\operatorname{Sp}(n, \mathbb{R}), 38$

unitário $\mathrm{U}(n), 38$

homomorfismo

de álgebras de Lie, 44

de grupos de Lie, 38

extensão de, 45

ideal, 41

identidade de Jacobi, 24

isomorfismo

de álgebras de Lie, 44

de grupos de Lie, 38

normalizador, 44, 70

produto

direto de grupos de Lie, 37 semi-direto

de álgebras de Lie, 46

de grupos de Lie, 38

sistema

controlável, 57

de controle, 30

dinâmico, 29

linear em espaço Euclideano, 53

linear em grupo de Lie, 71

linear localmente controlável, 97

simétrico, 88

transitivo, 88

subálgebra de Lie, 39

subgrupo de Lie, 37

teorema

de Kalman, 62

de Markus, 94

translação

à direita, 36

à esquerda, 36

variedade diferenciável, 18 